\title{
Heavy Neutral and Anionic Pnictogen
}

\section{Thiocyanates}

Sören Arlt, ${ }^{\dagger}$ Jörg Harloff, ${ }^{\dagger}$ Axel Schulz, ${ }^{*}+{ }^{+}$Alrik Stoffers ${ }^{\dagger}$ and Alexander Villinger ${ }^{\dagger}$

† Institut für Chemie, Universität Rostock, Albert-Einstein-Straße 3a, 18059 Rostock.

Leibniz-Institut für Katalyse an der Universität Rostock, Albert-Einstein-Straße 29a, 18059

Rostock.

1. Experimental p.2

2. Structure Elucidation p.3

3. Syntheses of Compounds $\quad$ p.26

4. Additional Analytical Data p.30

$\begin{array}{ll}\text { 5. References } & \text { p.51 }\end{array}$ 


\section{Experimental}

General Information. All manipulations were carried out under oxygen- and moisture-free conditions under argon using standard Schlenk or Drybox techniques.

Acetonitrile was dried over $\mathrm{CaH}_{2}$ and freshly distilled prior to use. Tetrahydrofuran (thf) was dried over $\mathrm{Na} /$ benzophenone and freshly distilled prior to use. $n$-Pentane was dried over $\mathrm{Na} /$ benzophenone/tetraglyme (tetraglyme $=\mathrm{Me}\left(\mathrm{OCH}_{2} \mathrm{CH}_{2}\right)_{3} \mathrm{OMe}$ ) and freshly distilled prior to use. Trimethylsilyl isothiocyanate (ABCR) was freshly distilled and degassed prior to use. $\mathrm{SbF}_{3}$ (Fluka) and $\mathrm{BiF}_{3}(\mathrm{ABCR})$ were used as received.

NMR spectra were obtained on a Bruker AVANCE 250, 300 or $500 \mathrm{MHz}$ spectrometer and were referenced internally to the deuterated solvent $\left({ }^{13} \mathrm{C}: c \mathrm{~d}_{3} c \mathrm{n} \delta_{\text {ref }}=118.3,1.3\right.$; thf- $d 8 \delta_{\text {ref }}=$ 67.6, $25.4 \mathrm{ppm})$, to protic impurities in the deuterated solvent $\left({ }^{1} \mathrm{H}: \mathrm{c} d_{3} \mathrm{cn} \delta_{\text {ref }}=2.13,1.94\right.$; thf$\left.d 8 \delta_{\text {ref }}=3.58,1.73 \mathrm{ppm}\right)$ or externally $\left({ }^{31} \mathrm{P}: 85 \% \mathrm{H}_{3} \mathrm{PO}_{4} \delta_{\text {ref }}=0 \mathrm{ppm}\right)$. All measurements were carried out at ambient temperature.

Raman spectra were recorded using a LabRAM HR 800 Horiba Jobin YVON Raman spectrometer equipped with an Olympus BX41 microscope with variable lenses. The samples were excited by a red laser $(633 \mathrm{~nm}, 17 \mathrm{~mW})$. All measurements were carried out at ambient temperature.

IR spectra were recorded on a Nicolet 380 FT-IR spectrometer with a Smart Orbit ATR unit at ambient temperature.

Elemental analyses were obtained using a vario Micro Cube from Elementar.

Melting points (uncorrected) were determined using a Stanford Research Systems EZ Melt at a heating rate of $20 \mathrm{~K} / \mathrm{min}$.

TGA/DSC were recorded on a Setaram Labsys TGA / DSC 1600.

Mass spectra were recorded on a Finnigan MAT 95-XP (Thermo Electron). 


\section{Structure Elucidation}

X-Ray quality crystals were selected in Fomblin YR-1800 perfluoroether (Alfa Aesar) at ambient temperature. The samples were cooled to 123(2) K during measurement. The data were collected on a Bruker Apex Kappa II CCD diffractometer using graphite monochromated Mo $\mathrm{K}_{\alpha}$ radiation $(\lambda=0.71073 \AA$ ). The structures were solved by direct methods (SHELXS$2013)^{[1]}<\sup >[1]</$ sup $>$ and refined by full matrix least squares procedures (SHELXL-2013). ${ }^{[2]}$ Semi-empirical absorption corrections were applied (SADABS). ${ }^{[3]}$ All non-hydrogen atoms were refined anisotropically, hydrogen atoms were included in the refinement at calculated positions using a riding model.

\section{$[\mathrm{PPN}][\mathrm{SCN}]$ :}

The SCN anion was found to be disorderd over two positions. The occupancy of each part was refined freely $(0.500 / 0.500)$.

PROBLEM: Short Inter D...A Contact S1.. N1.. 0.34 A ng.

RESPONSE: The atoms belong to different parts of a half-occupied, disordered NCS unit and are therefore not bound to each other.

$[\mathrm{PPN}]\left[\mathrm{H}(\mathrm{NCS})_{2}\right]$ :

PROBLEM: Suspect N-H Bond in CIF: N3 - H2. $1.35 \AA$ ng.

RESPONSE: May be considered as normal in this type of compound.

PROBLEM: Long N-H (N0.87, N1.01 ^) N1 - H1. $1.29 \AA \quad$ ng.

RESPONSE: May be considered as normal in this type of compound.

$[\mathrm{PPN}][\mathrm{NCS}] \cdot$ thf:

The thf was found to be disorderd over three positions. The occupancy of each part was refined freely $(0.439(2) / 0.323(3) / 0.238(2))$.

$\mathrm{Sb}(\mathrm{NCS})_{3} \cdot$ thf:

The thf was found to be disorderd over two positions. The occupancy of each part was refined freely $(0.648(8) / 0.352(8))$.

PROBLEM: Check Calcd Positive Residual Density on Sb1 2.02 e A-3

RESPONSE: Most probably caused by absorption effects. 


\section{$\mathrm{Bi}(\mathrm{NCS})_{3} \cdot$ thf:}

The thf was found to be disorderd over two positions. The occupancy of each part was refined freely $(0.76(2) / 0.24(2))$. 
Table S1. Crystallographic details of [PPN]SCN 1a, [PPN]SCN · thf 1b, [PPN][H(NCS $\left.)_{2}\right] 2$.

\begin{tabular}{|c|c|c|c|}
\hline Compound & {$[\mathrm{PPN}] \mathrm{SCN}$} & {$[\mathrm{PPN}] \mathrm{SCN} \cdot$ thf } & {$[\mathrm{PPN}]\left[\mathrm{H}(\mathrm{NCS})_{2}\right]$} \\
\hline Chem. Formula & $\mathrm{C}_{37} \mathrm{H}_{30} \mathrm{P}_{2} \mathrm{SN}_{2}$ & $\mathrm{C}_{41} \mathrm{H}_{38} \mathrm{P}_{2} \mathrm{OSN}_{2}$ & $\mathrm{C}_{38} \mathrm{H}_{31} \mathrm{P}_{2} \mathrm{~S}_{2} \mathrm{~N}_{3}$ \\
\hline Formula weight $[\mathrm{g} / \mathrm{mol}]$ & 596.63 & 668.73 & 655.72 \\
\hline Colour & colourless & colourless & colourless \\
\hline Crystal system & orthorhombic & triclinic & triclinic \\
\hline Space group & $\mathrm{Pbcn}$ & $\mathrm{P} 1$ & $\mathrm{P} 1$ \\
\hline$a[\AA]$ & $11.7102(3)$ & $9.4920(4)$ & $10.0035(9)$ \\
\hline$b[\AA]$ & $16.6086(5)$ & $10.8120(5)$ & $10.5636(9)$ \\
\hline$c[\AA]$ & $15.4661(4)$ & $16.9685(9)$ & $33.807(3)$ \\
\hline$\alpha\left[^{\circ}\right]$ & 90 & $92.678(2)$ & $91.879(3)$ \\
\hline$\beta\left[^{\circ}\right]$ & 90 & $94.182(2)$ & $94.279(3)$ \\
\hline$\gamma\left[{ }^{\circ}\right]$ & 90 & $91.777(2)$ & $105.567(3)$ \\
\hline$V\left[\AA^{3}\right]$ & $3008.0(2)$ & $1733.9(2)$ & $3426.7(5)$ \\
\hline $\mathrm{Z}$ & 4 & 2 & 4 \\
\hline$\rho_{\text {calc. }}\left[\mathrm{g} / \mathrm{cm}^{3}\right]$ & 1.317 & 1.281 & 1.271 \\
\hline$\mu\left[\mathrm{mm}^{-1}\right]$ & 0.24 & 0.22 & 0.28 \\
\hline$T[\mathrm{~K}]$ & 123 & 123 & 123 \\
\hline Measured reflections & 30048 & 86230 & 183938 \\
\hline Independent reflections & 5422 & 12028 & 18219 \\
\hline Reflections with $I>2 \sigma(I)$ & 4338 & 8461 & 13369 \\
\hline$R_{\text {int }}$ & 0.047 & 0.075 & 0.086 \\
\hline$F(000)$ & 1248 & 704 & 1368 \\
\hline$R_{1}\left(R\left[F^{2}>2 \sigma\left(F^{2}\right)\right]\right)$ & 0.044 & 0.059 & 0.061 \\
\hline $\mathrm{w} R_{2}\left(F^{2}\right)$ & 0.109 & 0.138 & 0.120 \\
\hline GooF & 1.06 & 1.03 & 1.11 \\
\hline No. of Parameters & 204 & 432 & 819 \\
\hline CCDC \# & 1895147 & 1895148 & 1895145 \\
\hline
\end{tabular}


Table S2. Crystallographic details of $\mathrm{Sb}(\mathrm{NCS})_{3} \mathbf{3 a}, \mathrm{Sb}(\mathrm{NCS})_{3} \cdot$ thf $\mathbf{3 b}, \mathrm{Bi}(\mathrm{NCS})_{3} \cdot$ thf 4 .

\begin{tabular}{|c|c|c|c|}
\hline Compound & $\begin{array}{l}\mathrm{Sb}(\mathrm{NCS})_{3} * 4 \% \\
\mathrm{Sb}(\mathrm{NCS})_{2} \mathrm{Cl}^{\mathrm{a}}\end{array}$ & $\mathrm{Sb}(\mathrm{NCS}) 3 \cdot$ thf & $\mathrm{Bi}(\mathrm{NCS})_{3} \cdot$ thf \\
\hline Chem. Formula & $\begin{array}{l}0.96(\mathrm{C} 3 \mathrm{~N} 3 \mathrm{~S} 3 \mathrm{Sb}) \\
0.04(\mathrm{C} 2 \mathrm{Cl} \text { N2 S2 Sb) }\end{array}$ & $\mathrm{C}_{7} \mathrm{H}_{8} \mathrm{~N}_{3} \mathrm{OS}_{3} \mathrm{Sb}$ & $\mathrm{C}_{7} \mathrm{H}_{8} \mathrm{BiN}_{3} \mathrm{OS}_{3}$ \\
\hline Formula weight $[\mathrm{g} / \mathrm{mol}]$ & 295.18 & 368.09 & 455.32 \\
\hline Colour & colourless & colourless & yellow \\
\hline Crystal system & tetragonal & monoclinic & monoclinic \\
\hline Space group & $\mathrm{I} 4{ }_{1} \mathrm{~cd}$ & $\mathrm{P} 2{ }_{1} / \mathrm{n}$ & $\mathrm{P} 2{ }_{1} / \mathrm{c}$ \\
\hline$a[\AA]$ & $16.7368(9)$ & $10.1535(4)$ & $6.6883(3)$ \\
\hline$b[\AA]$ & & $9.5123(4)$ & $9.2528(4)$ \\
\hline$c[\AA]$ & $11.6834(6)$ & $13.3497(6)$ & $20.1767(7)$ \\
\hline$\alpha\left[^{\circ}\right]$ & 90 & 90 & 90 \\
\hline$\beta\left[^{\circ}\right]$ & 90 & $102.080(2)$ & $90.131(2)$ \\
\hline$\gamma\left[{ }^{\circ}\right]$ & 90 & 90 & 90 \\
\hline$V\left[\AA^{3}\right]$ & $3272.8(4)$ & $1260.80(9)$ & $1248.64(9)$ \\
\hline $\mathrm{Z}$ & 16 & 4 & 4 \\
\hline$\rho_{\text {calc. }}\left[\mathrm{g} / \mathrm{cm}^{3}\right]$ & 2.396 & 1.939 & 2.422 \\
\hline$\mu\left[\mathrm{mm}^{-1}\right]$ & 4.06 & 2.66 & 14.60 \\
\hline$T[\mathrm{~K}]$ & 123 & 123 & $123(2)$ \\
\hline Measured reflections & 42857 & 38724 & 78264 \\
\hline Independent reflections & 2941 & 5566 & 4312 \\
\hline Reflections with $I>2 \sigma(I)$ & 2842 & 4307 & 3915 \\
\hline$R_{\text {int }}$ & 0.035 & 0.056 & 0.059 \\
\hline$F(000)$ & 2201 & 712 & 840 \\
\hline$R_{1}\left(R\left[F^{2}>2 \sigma\left(F^{2}\right)\right]\right)$ & 0.015 & 0.047 & 0.0401 \\
\hline $\mathrm{w} R_{2}\left(F^{2}\right)$ & 0.028 & 0.071 & 0.0606 \\
\hline GooF & 1.13 & 1.14 & 1.254 \\
\hline No. of Parameters & 101 & 182 & 183 \\
\hline CCDC \# & 1895150 & 1895151 & 1895149 \\
\hline
\end{tabular}

${ }^{a} \mathrm{Cl}$ impurities were found in the $\mathrm{Me}_{3} \mathrm{SiSCN}$ bought from ABCR. 
Table S3. Crystallographic details of $[\mathrm{PPN}]_{2}\left[\mathrm{Sb}(\mathrm{NCS})_{5}\right] \mathbf{5},[\mathrm{PPN}]_{2}\left[\mathrm{Bi}(\mathrm{NCS})_{3}(\mathrm{SCN})_{2}(\right.$ thf $\left.)\right] \mathbf{6}$.

\begin{tabular}{|c|c|c|}
\hline Compound & {$[\mathrm{PPN}]_{2}\left[\mathrm{Sb}(\mathrm{NCS})_{5}\right]$} & {$[\mathrm{PPN}]_{2}\left[\mathrm{Bi}(\mathrm{NCS})_{3}(\mathrm{SCN})_{2}(\mathrm{thf})\right]$} \\
\hline Chem. Formula & $\mathrm{C}_{77} \mathrm{H}_{60} \mathrm{~N}_{7} \mathrm{P}_{4} \mathrm{~S}_{5} \mathrm{Sb}$ & $\mathrm{C}_{81} \mathrm{H}_{68} \mathrm{P}_{4} \mathrm{~N}_{7} \mathrm{~S}_{5} \mathrm{OBi}$ \\
\hline Formula weight $[\mathrm{g} / \mathrm{mol}]$ & 1489.25 & 1648.58 \\
\hline Colour & colourless & yellow \\
\hline Crystal system & monoclinic & monoclinic \\
\hline Space group & $\mathrm{P} 2{ }_{1} / \mathrm{c}$ & $\mathrm{P} 2{ }_{1}$ \\
\hline$a[\AA]$ & $21.4216(10)$ & $9.8913(4)$ \\
\hline$b[\AA]$ & $18.9556(9)$ & $19.779(1)$ \\
\hline$c[\AA]$ & $17.4019(8)$ & $19.165(1)$ \\
\hline$\alpha\left[^{\circ}\right]$ & 90 & 90 \\
\hline$\beta\left[^{\circ}\right]$ & $91.283(2)$ & $98.823(2)$ \\
\hline$\gamma\left[{ }^{\circ}\right]$ & 90 & 90 \\
\hline$V\left[\AA^{3}\right]$ & $7064.4(6)$ & $3705.0(3)$ \\
\hline $\mathrm{Z}$ & 4 & 2 \\
\hline$\rho_{\text {calc. }}\left[\mathrm{g} / \mathrm{cm}^{3}\right]$ & 1.400 & 1.478 \\
\hline$\mu\left[\mathrm{mm}^{-1}\right]$ & 0.68 & 2.66 \\
\hline$T[\mathrm{~K}]$ & 123 & 123 \\
\hline Measured reflections & 69068 & 127418 \\
\hline Independent reflections & 12476 & 21594 \\
\hline Reflections with $I>2 \sigma(I)$ & 8558 & 17769 \\
\hline$R_{\text {int }}$ & 0.112 & 0.091 \\
\hline$F(000)$ & 3048 & 1668 \\
\hline$R_{1}\left(R\left[F^{2}>2 \sigma\left(F^{2}\right)\right]\right)$ & 0.040 & 0.044 \\
\hline $\mathrm{w} R_{2}\left(F^{2}\right)$ & 0.086 & 0.077 \\
\hline GooF & 1.01 & 1.01 \\
\hline No. of Parameters & 847 & 893 \\
\hline CCDC \# & 1895146 & 1895145 \\
\hline
\end{tabular}


Scheme S1. Numbering scheme of [PPN]SCN 1a.

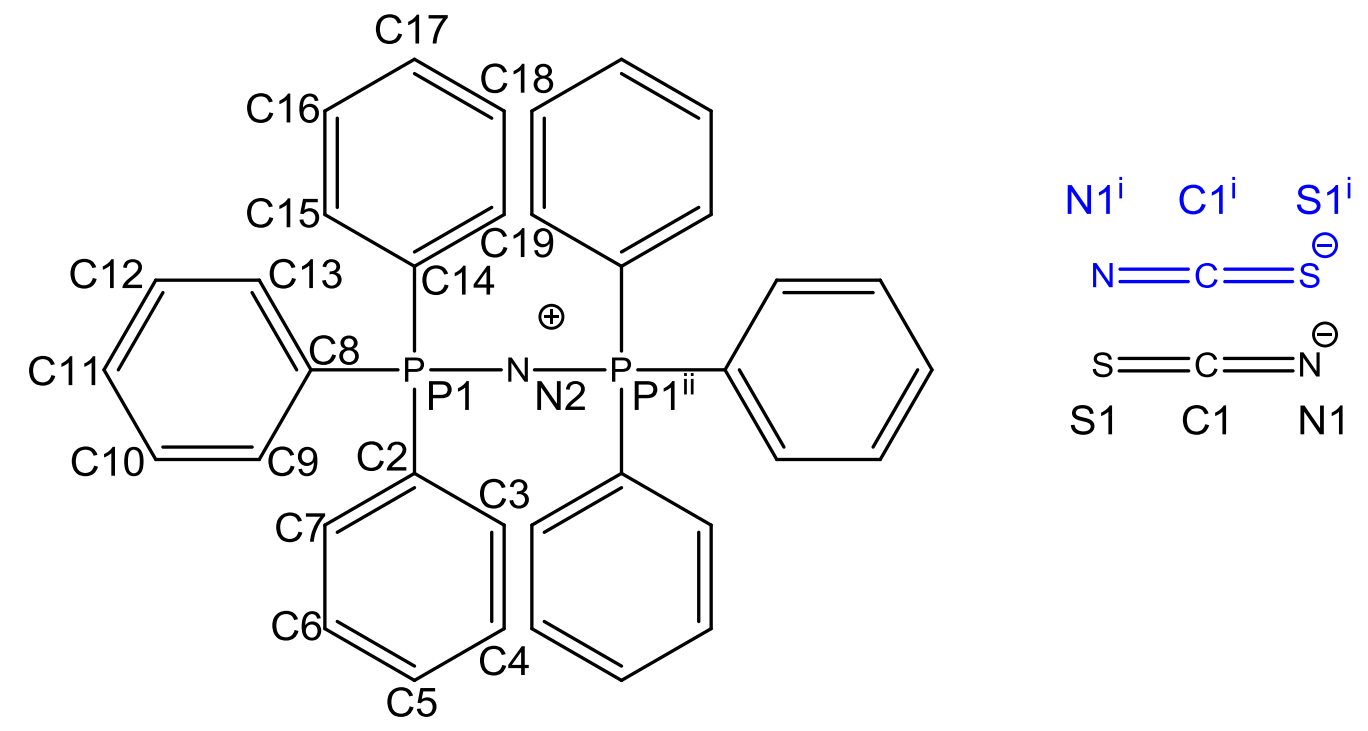

Table S4. Selected bond lengths $(\AA)$, distances $(\AA)$ and angles $\left(^{\circ}\right)$ of $[\mathrm{PPN}] \mathrm{SCN} 1 \mathrm{a}$.

\begin{tabular}{llll}
\hline $\mathrm{S} 1-\mathrm{N} 1^{\mathrm{i}}$ & $0.335(7)$ & $\mathrm{C} 3-\mathrm{C} 4$ & $1.385(2)$ \\
$\mathrm{S} 1-\mathrm{C} 1^{\mathrm{i}}$ & $0.850(4)$ & $\mathrm{C} 4-\mathrm{C} 5$ & $1.390(2)$ \\
$\mathrm{S} 1-\mathrm{C} 1$ & $1.614(4)$ & $\mathrm{C} 5-\mathrm{C} 6$ & $1.384(2)$ \\
$\mathrm{S} 1-\mathrm{S} 1^{\mathrm{i}}$ & $2.463(4)$ & $\mathrm{C} 6-\mathrm{C} 7$ & $1.391(2)$ \\
$\mathrm{N} 1-\mathrm{S} 1^{\mathrm{i}}$ & $0.335(7)$ & $\mathrm{C} 8-\mathrm{C} 13$ & $1.394(2)$ \\
$\mathrm{N} 1-\mathrm{C} 1$ & $1.157(6)$ & $\mathrm{C} 8-\mathrm{C} 9$ & $1.402(2)$ \\
$\mathrm{N} 1-\mathrm{C} 1^{\mathrm{i}}$ & $1.923(9)$ & $\mathrm{C} 9-\mathrm{C} 10$ & $1.389(2)$ \\
$\mathrm{C} 1-\mathrm{C} 1^{\mathrm{i}}$ & $0.767(7)$ & $\mathrm{C} 10-\mathrm{C} 11$ & $1.386(2)$ \\
$\mathrm{C} 1-\mathrm{S} 1^{\mathrm{i}}$ & $0.850(4)$ & $\mathrm{C} 11-\mathrm{C} 12$ & $1.389(2)$ \\
$\mathrm{C} 1-\mathrm{N} 1^{\mathrm{i}}$ & $1.923(9)$ & $\mathrm{C} 12-\mathrm{C} 13$ & $1.391(2)$ \\
$\mathrm{P} 1-\mathrm{N} 2$ & $1.5765(5)$ & $\mathrm{C} 14-\mathrm{C} 15$ & $1.398(2)$ \\
$\mathrm{P} 1-\mathrm{C} 14$ & $1.794(2)$ & $\mathrm{C} 14-\mathrm{C} 19$ & $1.398(2)$ \\
$\mathrm{P} 1-\mathrm{C} 2$ & $1.795(2)$ & $\mathrm{C} 15-\mathrm{C} 16$ & $1.389(2)$ \\
$\mathrm{P} 1-\mathrm{C} 8$ & $1.800(2)$ & $\mathrm{C} 16-\mathrm{C} 17$ & $1.387(2)$ \\
$\mathrm{N} 2-\mathrm{P} 1^{\mathrm{ii}}$ & $1.5765(5)$ & $\mathrm{C} 17-\mathrm{C} 18$ & $1.391(2)$ \\
$\mathrm{C} 2-\mathrm{C} 7$ & $1.393(2)$ & $\mathrm{C} 18-\mathrm{C} 19$ & $1.393(2)$ \\
\hline
\end{tabular}




\begin{tabular}{|c|c|c|c|}
\hline C2-C3 & $1.400(2)$ & & \\
\hline $\mathrm{N} 1-\mathrm{S} 1-\mathrm{C} 1^{\mathrm{i}}$ & $152(2)$ & $\mathrm{P} 1-\mathrm{N} 2-\mathrm{P} 1^{\mathrm{ii}}$ & $144.0(1)$ \\
\hline N1i-S1-C1 & $155(2)$ & C7-C2-C3 & $119.8(2)$ \\
\hline $\mathrm{C} 1 \mathrm{i}-\mathrm{S} 1-\mathrm{C} 1$ & $3.4(3)$ & C7-C2-P1 & $122.45(9)$ \\
\hline$N 1^{i-S 1-S 1} 1^{i}$ & $155(2)$ & C3-C2-P1 & $117.57(9)$ \\
\hline $\mathrm{C} 1^{\mathrm{i}-\mathrm{S}} 1-\mathrm{S} 1^{\mathrm{i}}$ & $2.7(3)$ & C4-C3-C2 & $112.0(2)$ \\
\hline $\mathrm{C} 1-\mathrm{S} 1-\mathrm{S} 1^{\mathrm{i}}$ & $1.4(2)$ & C3-C4-C5 & $120.0(2)$ \\
\hline S1-N1-C1 & $20(2)$ & C6-C5-C4 & $120.2(2)$ \\
\hline 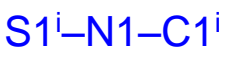 & $20(2)$ & C5-C6-C7 & $120.2(2)$ \\
\hline $\mathrm{C} 1-\mathrm{N} 1-\mathrm{C} 1^{\mathrm{i}}$ & $1.4(2)$ & C6-C7-C2 & $119.8(2)$ \\
\hline $\mathrm{C} 1-\mathrm{C} 1-\mathrm{S} 1^{\mathrm{i}}$ & $172.9(7)$ & C13-C8-C9 & $119.6(2)$ \\
\hline C1-C1-N1 & $176.6(6)$ & C13-C8-P1 & $119.86(9)$ \\
\hline $\mathrm{S} 1 \mathrm{i}-\mathrm{C} 1-\mathrm{N} 1$ & $7.8(6)$ & C9-C8-P1 & $120.45(9)$ \\
\hline $\mathrm{C} 1-\mathrm{C} 1-\mathrm{S} 1$ & $3.7(4)$ & C10-C9-C8 & $119.7(2)$ \\
\hline $\mathrm{S} 1{ }^{\mathrm{i}-\mathrm{C} 1-\mathrm{S} 1}$ & $175.9(4)$ & C11-C10-C9 & $120.5(2)$ \\
\hline $\mathrm{N} 1-\mathrm{C} 1-\mathrm{S} 1$ & $176.3(6)$ & C10-C11-C12 & $119.9(2)$ \\
\hline 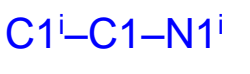 & $2.1(3)$ & C11-C12-C13 & $120.1(2)$ \\
\hline $\mathrm{S} 11^{\mathrm{i}} \mathrm{C} 1-\mathrm{N} 1^{\mathrm{i}}$ & $173.6(5)$ & C12-C13-C8 & $120.1(2)$ \\
\hline $\mathrm{N} 1-\mathrm{C} 1-\mathrm{N} 1^{\mathrm{i}}$ & $174.7(9)$ & C15-C14-C19 & $120.1(2)$ \\
\hline $\mathrm{S} 1-\mathrm{C} 1-\mathrm{N} 1^{\mathrm{i}}$ & $4.2(3)$ & C15-C14-P1 & $118.4(1)$ \\
\hline N2-P1-C14 & $108.47(6)$ & C19-C14-P1 & $121.4(1)$ \\
\hline N2-P1-C2 & $114.49(6)$ & C16-C15-C14 & $119.7(2)$ \\
\hline C14-P1-C2 & $108.29(6)$ & C17-C16-C15 & $120.3(2)$ \\
\hline N2-P1-C8 & $110.18(5)$ & C16-C17-C18 & $120.3(2)$ \\
\hline C14-P1-C8 & $105.62(5)$ & C17-C18-C19 & $119.9(2)$ \\
\hline C2-P1-C8 & $109.39(5)$ & C18-C19-C14 & $119.7(2)$ \\
\hline
\end{tabular}

Symmetry codes: (i) $-x+1, y,-z+3 / 2$; (ii) $-x+2, y,-z+3 / 2$. 
Figure S1. ORTEP representation of [PPN]SCN 1a. Thermal ellipsoids with $50 \%$ probability at $123 \mathrm{~K}, \mathrm{H}$ atoms are omitted for clarity.

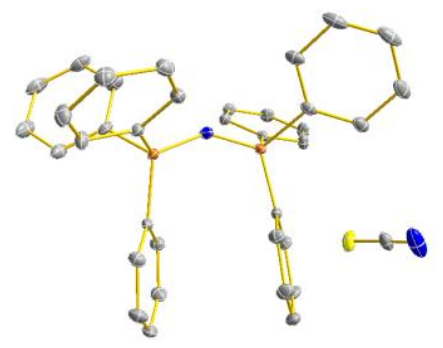


Scheme S2. Numbering scheme of [PPN]NCS $\cdot$ thf $\mathbf{1 b}$.
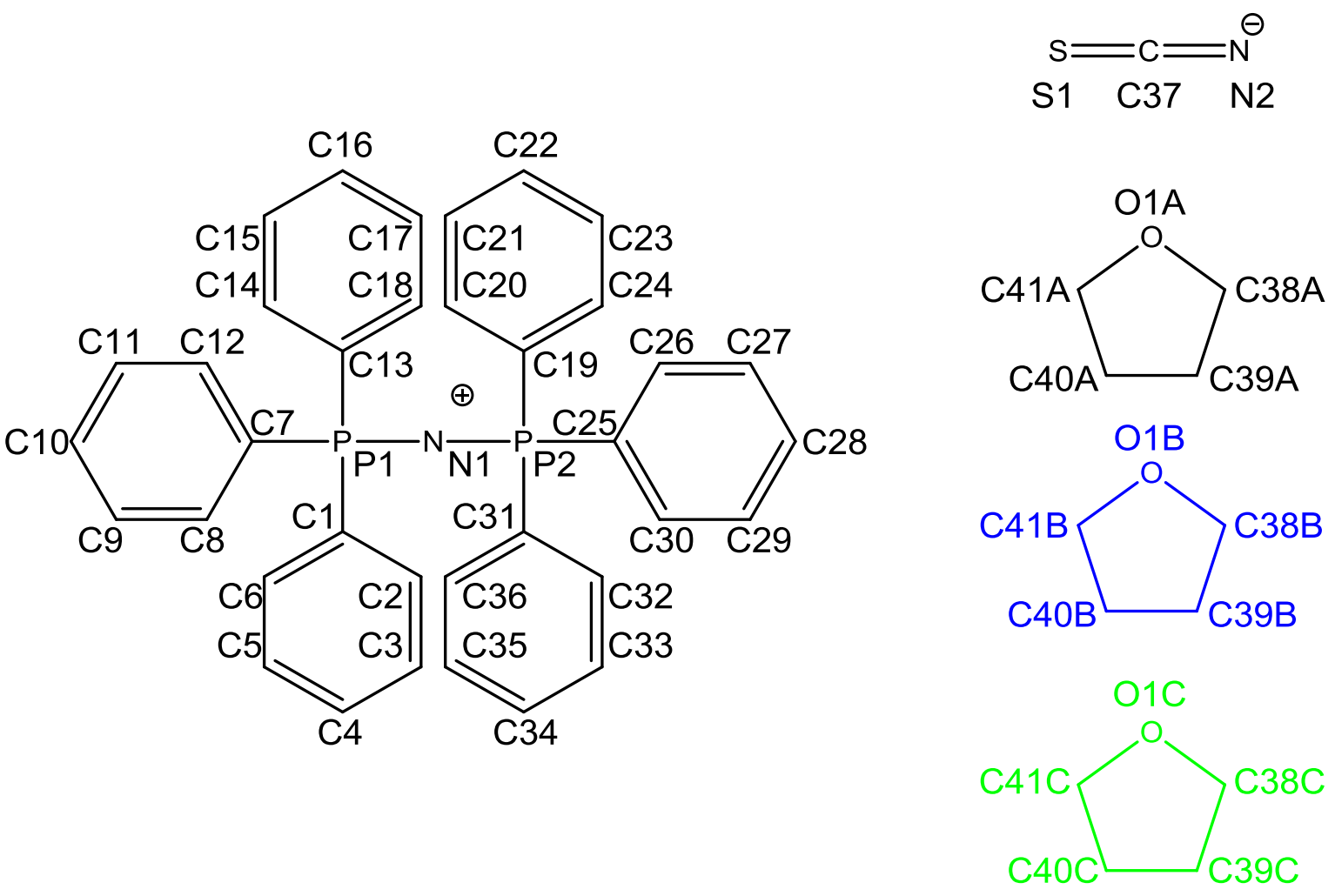

Table S5. Selected bond lengths $(\AA)$, distances $(\AA)$ and angles $\left(^{\circ}\right)$ of $[\mathrm{PPN}] \mathrm{NCS} \cdot \operatorname{thf} \mathbf{1 b}$.

\begin{tabular}{llll}
\hline O1A-C41A & $1.384(6)$ & C7-C12 & $1.388(3)$ \\
O1A-C38A & $1.548(6)$ & C7-C8 & $1.399(2)$ \\
C38A-C39A & $1.497(7)$ & C8-C9 & $1.382(3)$ \\
C39A-C40A & $1.503(8)$ & C9-C10 & $1.385(3)$ \\
C40A-C41A & $1.468(7)$ & C10-C11 & $1.381(3)$ \\
O1B-C41B & $1.406(7)$ & C11-C12 & $1.395(3)$ \\
O1B-C38B & $1.576(7)$ & C13-C18 & $1.396(3)$ \\
C38B-C39B & $1.484(8)$ & C13-C14 & $1.399(3)$ \\
C39B-C40B & $1.485(9)$ & C14-C15 & $1.390(3)$ \\
C40B-C41B & $1.458(8)$ & C15-C16 & $1.382(4)$ \\
O1C-C41C & $1.384(8)$ & C16-C17 & $1.379(4)$ \\
O1C-C38C & $1.571(8)$ & C17-C18 & $1.393(3)$ \\
C38C-C39C & $1.488(9)$ & C19-C20 & $1.397(2)$ \\
C39C-C40C & $1.491(9)$ & C19-C24 & $1.400(2)$ \\
C40C-C41C & $1.451(9)$ & C20-C21 & $1.392(3)$ \\
S1-C37 & $1.642(2)$ & C21-C22 & $1.389(3)$ \\
\hline
\end{tabular}




\begin{tabular}{llll}
\hline N2-C37 & $1.151(3)$ & C22-C23 & $1.390(3)$ \\
P1-N1 & $1.583(2)$ & C23-C24 & $1.385(3)$ \\
P1-C7 & $1.797(2)$ & C25-C26 & $1.388(3)$ \\
P1-C13 & $1.797(2)$ & C25-C30 & $1.395(3)$ \\
P1-C1 & $1.803(2)$ & C26-C27 & $1.390(3)$ \\
P2-N1 & $1.583(2)$ & C27-C28 & $1.383(3)$ \\
P2-C19 & $1.794(2)$ & C28-C29 & $1.381(3)$ \\
P2-C31 & $1.794(2)$ & C29-C30 & $1.390(3)$ \\
P2-C25 & $1.804(2)$ & C31-C32 & $1.389(2)$ \\
C1-C6 & $1.396(2)$ & C31-C36 & $1.399(2)$ \\
C1-C2 & $1.397(2)$ & C32-C33 & $1.389(3)$ \\
C2-C3 & $1.387(3)$ & C33-C34 & $1.381(3)$ \\
C3-C4 & $1.386(3)$ & C34-C35 & $1.390(3)$ \\
C4-C5 & $1.386(3)$ & C35-C36 & $1.383(2)$ \\
C5-C6 & $1.394(3)$ & & \\
C41A-O1A-C38A & $108.4(5)$ & C8-C7-P1 & $120.2(2)$ \\
C39A-C38A-O1A & $104.1(4)$ & C9-C8-C7 & $120.0(2)$ \\
C38A-C39A-C40A & $103.1(5)$ & C8-C9-C10 & $120.0(2)$ \\
C41A-C40A-C39A & $105.2(4)$ & C11-C10-C9 & $120.4(2)$ \\
O1A-C41A-C40A & $107.8(5)$ & C10-C11-C12 & $120.1(2)$ \\
C41B-O1B-C38B & $105.9(5)$ & C7-C12-C11 & $119.6(2)$ \\
C39B-C38B-O1B & $106.0(5)$ & C18-C13-C14 & $119.4(2)$ \\
C38B-C39B-C40B & $104.7(6)$ & C18-C13-P1 & $121.3(2)$ \\
C41B-C40B-C39B & $107.3(5)$ & C14-C13-P1 & $118.9(2)$ \\
O1B-C41B-C40B & $109.3(6)$ & C15-C14-C13 & $120.1(2)$ \\
C41C-O1C-C38C & $106.7(6)$ & C16-C15-C14 & $119.9(2)$ \\
C39C-C38C-O1C & $103.6(6)$ & C17-C16-C15 & $120.6(2)$ \\
C38C-C39C-C40C & $106.6(7)$ & C16-C17-C18 & $120.0(2)$ \\
C41C-C40C-C39C & $106.1(6)$ & C17-C18-C13 & $120.0(2)$ \\
O1C-C41C-C40C & $107.7(7)$ & C20-C19-C24 & $120.4(2)$ \\
N2-C37-S1 & $178.5(2)$ & C20-C19-P2 & $119.6(2)$ \\
N1-P1-C7 & $107.66(8)$ & C24-C19-P2 & $120.0(2)$ \\
N1-P1-C13 & $114.45(8)$ & C21-C20-C19 & $119.4(2)$ \\
C7-P1-C13 & $107.17(8)$ & C22-C21-C20 & $120.1(2)$ \\
N1-P1-C1 & $112.33(8)$ & C21-C22-C23 & $120.4(2)$ \\
C7-P1-C1 & $107.07(8)$ & C24-C23-C22 & $120.3(2)$ \\
C13-P1-C1 & $107.79(8)$ & C23-C24-C19 & $119.5(2)$ \\
N1-P2-C19 & $109.06(8)$ & C26-C25-C30 & $119.5(2)$ \\
N1-P2-C31 & $113.89(8)$ & C26-C25-P2 & $122.1(2)$ \\
\hline
\end{tabular}




\begin{tabular}{llll}
\hline C19-P2-C31 & $106.81(8)$ & C30-C25-P2 & $118.5(2)$ \\
N1-P2-C25 & $110.46(8)$ & C25-C26-C27 & $119.9(2)$ \\
C19-P2-C25 & $107.73(8)$ & C28-C27-C26 & $120.4(2)$ \\
C31-P2-C25 & $108.66(8)$ & C29-C28-C27 & $120.2(2)$ \\
P1-N1-P2 & $137.7(2)$ & C28-C29-C30 & $119.7(2)$ \\
C6-C1-C2 & $119.8(2)$ & C29-C30-C25 & $120.4(2)$ \\
C6-C1-P1 & $119.7(2)$ & C32-C31-C36 & $119.6(2)$ \\
C2-C1-P1 & $120.5(2)$ & C32-C31-P2 & $122.7(2)$ \\
C3-C2-C1 & $119.8(2)$ & C36-C31-P2 & $117.5(2)$ \\
C4-C3-C2 & $120.5(2)$ & C31-C32-C33 & $119.8(2)$ \\
C5-C4-C3 & $120.0(2)$ & C34-C33-C32 & $120.4(2)$ \\
C4-C5-C6 & $120.2(2)$ & C33-C34-C35 & $120.2(2)$ \\
C5-C6-C1 & $119.7(2)$ & C36-C35-C34 & $119.7(2)$ \\
C12-C7-C8 & $119.9(2)$ & C35-C36-C31 & $120.3(2)$ \\
C12-C7-P1 & $119.8(2)$ & & \\
\hline
\end{tabular}

Figure S2. ORTEP representation of [PPN]NCS $\cdot$ thf $\mathbf{1 b}$. Thermal ellipsoids with 50\% probability at $123 \mathrm{~K}, \mathrm{H}$ atoms are omitted for clarity.
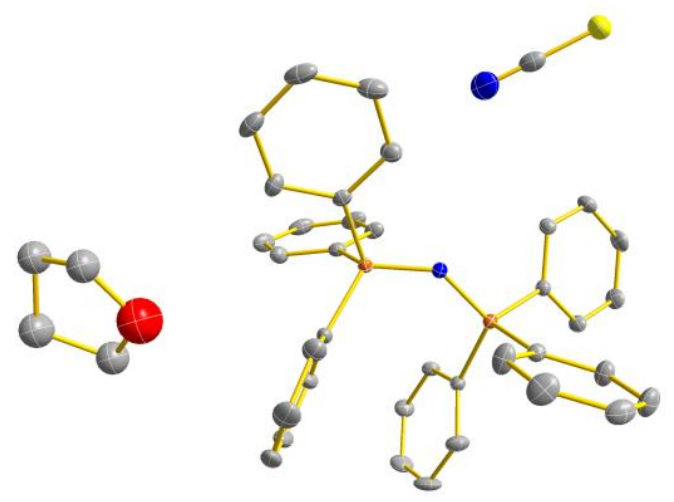
Scheme S3. Numbering scheme of $[\mathrm{PPN}]\left[\mathrm{H}(\mathrm{NCS})_{2}\right] 2$.

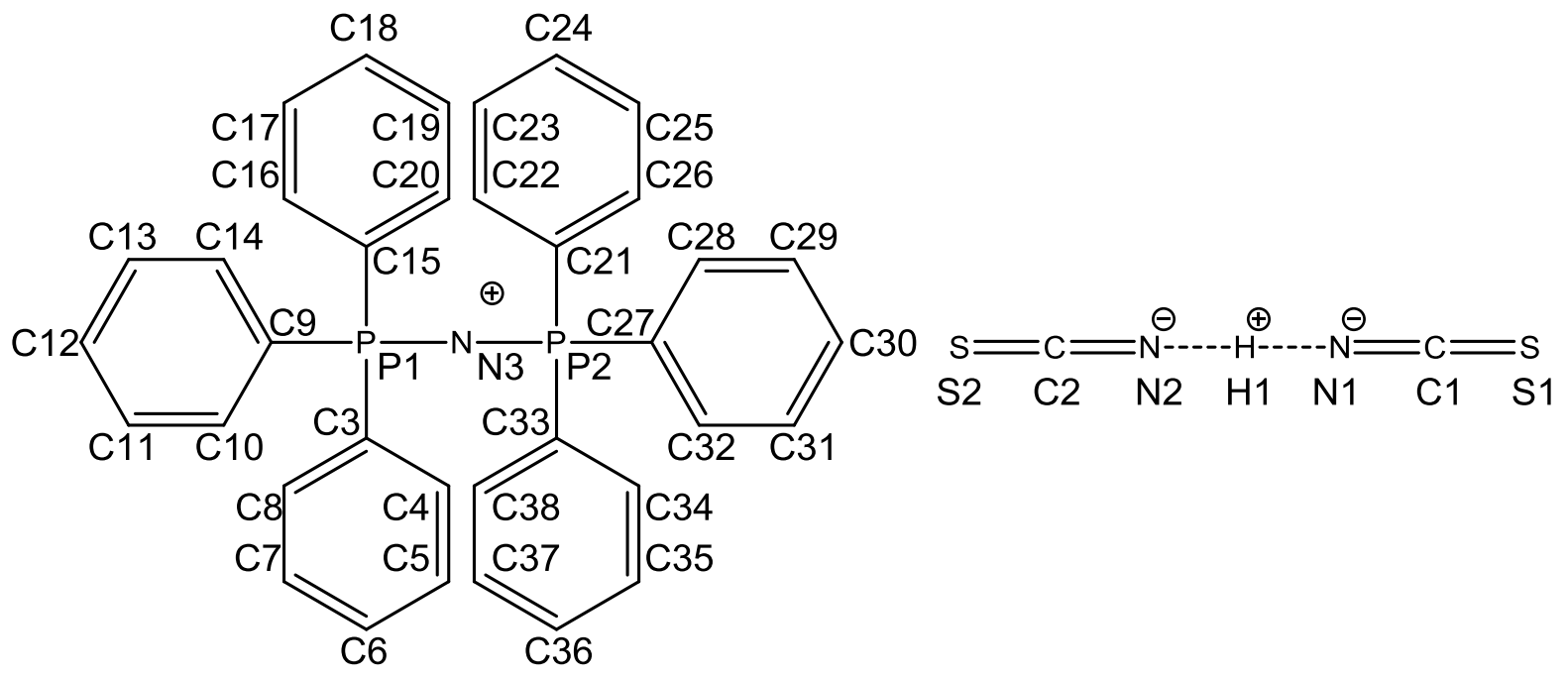

Table S6. Selected bond lengths $(\AA)$, distances $(\AA)$ and angles $\left(^{\circ}\right)$ of $[\mathrm{PPN}]\left[\mathrm{H}(\mathrm{NCS})_{2}\right] 2$.

\begin{tabular}{llll}
\hline S1-C1 & $1.615(5)$ & C13-C14 & $1.377(6)$ \\
S2-C2 & $1.606(5)$ & C15-C16 & $1.393(5)$ \\
N1-C1 & $1.155(6)$ & C15-C20 & $1.396(5)$ \\
N1-H1 & $1.33(5)$ & C16-C17 & $1.388(6)$ \\
N2-C2 & $1.155(6)$ & $\mathrm{C} 17-C 18$ & $1.376(6)$ \\
N2-H1 & $1.21(5)$ & C18-C19 & $1.386(6)$ \\
P1-N3 & $1.585(3)$ & $\mathrm{C} 19-C 20$ & $1.382(6)$ \\
P1-C3 & $1.794(4)$ & C21-C26 & $1.396(5)$ \\
P1-C9 & $1.796(4)$ & C21-C22 & $1.400(6)$ \\
P1-C15 & $1.798(4)$ & C22-C23 & $1.380(6)$ \\
P2-N3 & $1.579(3)$ & C23-C24 & $1.388(6)$ \\
P2-C21 & $1.795(4)$ & C24-C25 & $1.373(6)$ \\
P2-C33 & $1.798(4)$ & C25-C26 & $1.395(6)$ \\
P2-C27 & $1.804(4)$ & C27-C28 & $1.391(6)$ \\
C3-C8 & $1.392(5)$ & C27-C32 & $1.393(6)$ \\
C3-C4 & $1.399(6)$ & C28-C29 & $1.386(6)$ \\
C4-C5 & $1.376(6)$ & C29-C30 & $1.382(6)$
\end{tabular}




$\begin{array}{llll}\text { C5-C6 } & 1.375(7) & \text { C30-C31 } & 1.368(7) \\ \text { C6-C7 } & 1.376(7) & \text { C31-C32 } & 1.395(6) \\ \text { C7-C8 } & 1.383(6) & \text { C33-C38 } & 1.388(5) \\ \text { C9-C10 } & 1.392(5) & \text { C33-C34 } & 1.400(5) \\ \text { C9-C14 } & 1.397(5) & \text { C34-C35 } & 1.386(6) \\ \text { C10-C11 } & 1.388(6) & \text { C35-C36 } & 1.397(6) \\ \text { C11-C12 } & 1.384(6) & \text { C36-C37 } & 1.386(6) \\ \text { C12-C13 } & 1.384(6) & \text { C37-C38 } & 1.394(6) \\ \text { C1-N1-H1 } & 151(2) & \text { C16-C15-C20 } & 119.3(4) \\ \text { C2-N2-H1 } & 169(2) & \text { C16-C15-P1 } & 119.6(3) \\ \text { N1-C1-S1 } & 178.5(4) & \text { C20-C15-P1 } & 120.9(3) \\ \text { N2-C2-S2 } & 179.0(5) & \text { C17-C16-C15 } & 119.9(4) \\ \text { N3-P1-C3 } & 115.6(2) & \text { C18-C17-C16 } & 120.4(4) \\ \text { N3-P1-C9 } & 108.1(2) & \text { C17-C18-C19 } & 120.1(4) \\ \text { C3-P1-C9 } & 106.1(2) & \text { C20-C19-C18 } & 120.1(4) \\ \text { N3-P1-C15 } & 109.7(2) & \text { C19-C20-C15 } & 120.2(4) \\ \text { C3-P1-C15 } & 109.9(2) & \text { C26-C21-C22 } & 119.8(4) \\ \text { C9-P1-C15 } & 107.1(2) & \text { C26-C21-P2 } & 121.5(3) \\ \text { N3-P2-C21 } & 112.6(2) & \text { C22-C21-P2 } & 118.5(3) \\ \text { N3-P2-C33 } & 107.1(2) & \text { C23-C22-C21 } & 120.0(4) \\ \text { C21-P2-C33 } & 107.0(2) & \text { C22-C23-C24 } & 120.1(4) \\ \text { N3-P2-C27 } & 114.1(2) & \text { C25-C24-C23 } & 120.2(4) \\ \text { C21-P2-C27 } & 107.5(2) & \text { C24-C25-C26 } & 120.8(4) \\ \text { C33-P2-C27 } & 108.2(2) & \text { C25-C26-C21 } & 119.1(4) \\ \text { P2-N3-P1 } & 140.5(2) & \text { C28-C27-C32 } & 119.9(4) \\ \text { C8-C3-C4 } & 119.4(4) & \text { C28-C27-P2 } & 119.7(3) \\ \text { C8-C3-P1 } & 123.4(3) & \text { C32-C27-P2 } & 120.0(3) \\ \text { C4-C3-P1 } & 117.1(3) & \text { C29-C28-C27 } & 120.0(4) \\ \text { C5-C4-C3 } & 119.9(4) & \text { C30-29-C28 } & 119.9(4) \\ \text { C5-C6-C7-C4 } & 120.1(4) & \text { C31-C30-C29 } & 120.4(4) \\ & 120.7(4) & \text { C30-C31-C32 } & 120.6(5) \\ & & \text { S15 } & \\ \text { C2 } & & \end{array}$




\begin{tabular}{llll} 
C6-C7-C8 & $120.0(4)$ & C27-C32-C31 & $119.2(4)$ \\
C7-C8-C3 & $119.9(4)$ & C38-C33-C34 & $119.8(4)$ \\
C10-C9-C14 & $119.7(4)$ & C38-C33-P2 & $120.4(3)$ \\
C10-C9-P1 & $118.3(3)$ & C34-C33-P2 & $119.8(3)$ \\
C14-C9-P1 & $121.9(3)$ & C35-C34-C33 & $119.9(4)$ \\
C11-C10-C9 & $120.0(4)$ & C34-C35-C36 & $120.2(4)$ \\
C12-C11-C10 & $119.9(4)$ & C37-C36-C35 & $119.8(4)$ \\
C11-C12-C13 & $120.0(4)$ & C36-C37-C38 & $120.2(4)$ \\
C14-C13-C12 & $120.8(4)$ & C33-C38-C37 & $120.1(4)$ \\
C13-C14-C9 & $119.5(4)$ & & \\
\hline
\end{tabular}

Figure S3. ORTEP representation of $[\mathrm{PPN}]\left[\mathrm{H}(\mathrm{NCS})_{2}\right]$ 2. Thermal ellipsoids with $50 \%$ probability at $123 \mathrm{~K}, \mathrm{H}$ atoms are pictured as balls.
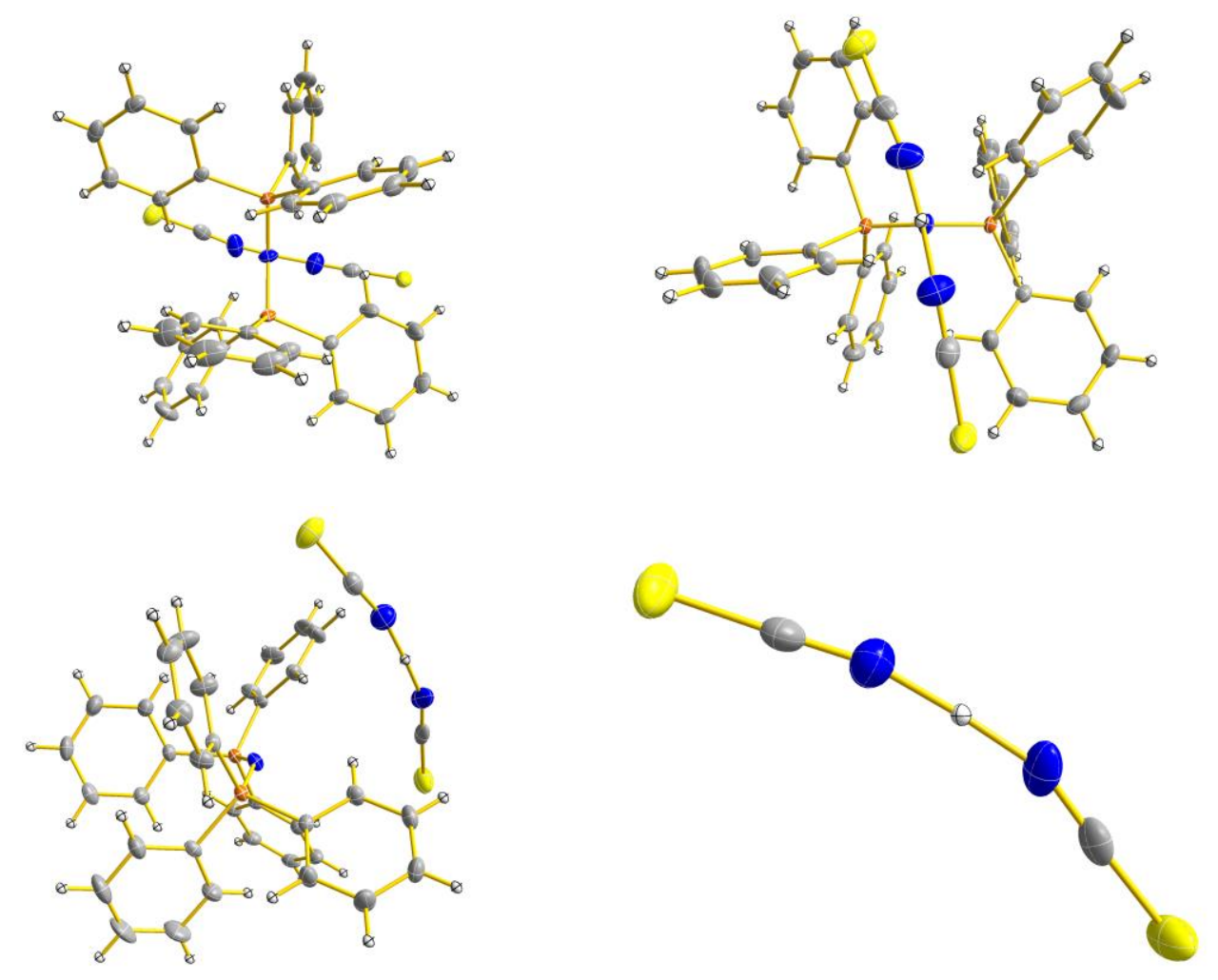
Scheme S4. Numbering scheme of $\mathrm{Sb}(\mathrm{NCS})_{3} 3 \mathbf{a}$.

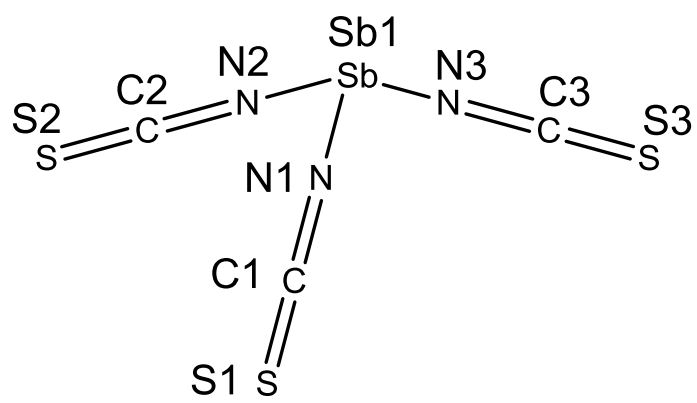

Table S7. Selected bond lengths $(\AA)$, distances $(\AA)$ and angles $\left(^{\circ}\right)$ of $\operatorname{Sb}(\mathrm{NCS})_{3} 3 \mathbf{a}$.

\begin{tabular}{llll}
\hline Sb1-N3 & $2.093(2)$ & N3-Sb1-N2 & $83.87(9)$ \\
Sb1-N2 & $2.099(2)$ & N3-Sb1-N1 & $85.6(1)$ \\
Sb1-N1 & $2.103(3)$ & N2-Sb1-N1 & $87.9(1)$ \\
S1-C1 & $1.600(3)$ & C1-N1-Sb1 & $138.9(3)$ \\
S2-C2 & $1.613(3)$ & C2-N2-Sb1 & $175.6(2)$ \\
S3-C3 & $1.607(3)$ & C3-N3-Sb1 & $141.6(2)$ \\
N1-C1 & $1.182(4)$ & N1-C1-S1 & $177.7(3)$ \\
N2-C2 & $1.165(4)$ & N2-C2-S2 & $179.0(3)$ \\
N3-C3 & $1.175(4)$ & N3-C3-S3 & $178.1(3)$ \\
\hline
\end{tabular}

Figure S4. ORTEP representation of $\mathrm{Sb}(\mathrm{NCS})_{3} 3 \mathbf{3}$. Thermal ellipsoids with $50 \%$ probability at $123 \mathrm{~K}, \mathrm{H}$ atoms are omitted for clarity.

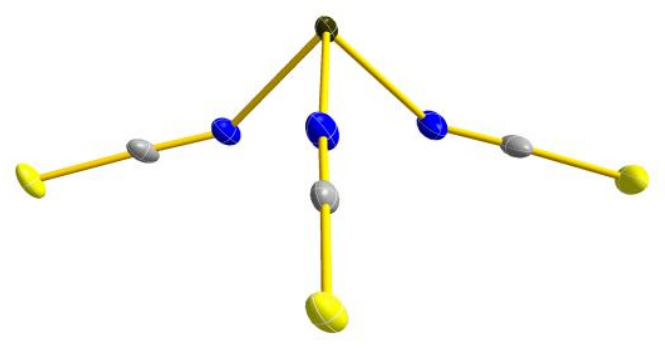


Scheme S5. Numbering scheme of $\mathrm{Sb}(\mathrm{NCS})_{3} \cdot$ thf $\mathbf{3 b}$.

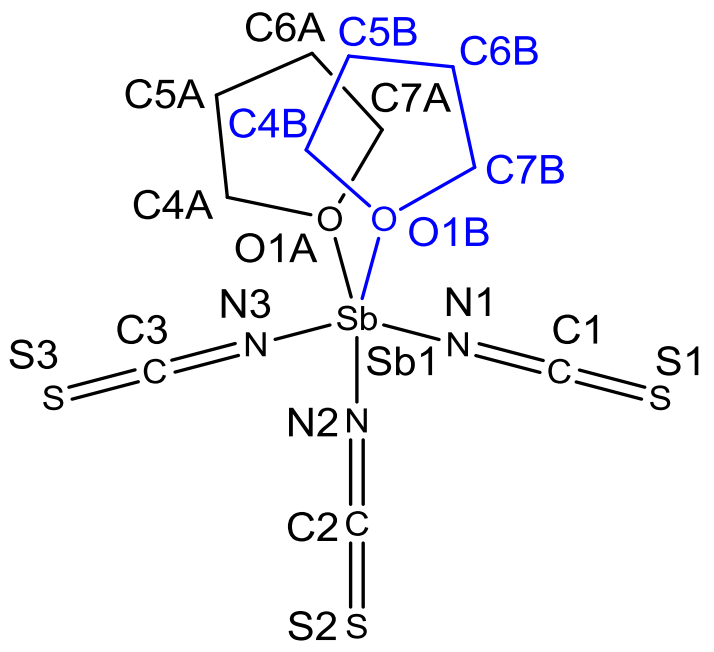

Table S8. Selected bond lengths $(\AA)$, distances $(\AA)$ and angles $\left(^{\circ}\right)$ of $\operatorname{Sb}(\mathrm{NCS})_{3} \cdot$ thf $\mathbf{3 b}$.

\begin{tabular}{llll}
\hline Sb1-N2 & $2.070(2)$ & O1A-C7A & $1.455(5)$ \\
Sb1-N3 & $2.101(3)$ & O1A-C4A & $1.474(6)$ \\
Sb1-N1 & $2.151(2)$ & C4A-C5A & $1.516(7)$ \\
Sb1-O1B & $2.403(9)$ & C5A-C6A & $1.533(9)$ \\
Sb1-O1A & $2.421(5)$ & C6A-C7A & $1.510(6)$ \\
S1-C1 & $1.610(3)$ & O1B-C7B & $1.458(7)$ \\
S2-C2 & $1.586(3)$ & O1B-C4B & $1.471(8)$ \\
S3-C3 & $1.600(3)$ & C4B-C5B & $1.519(9)$ \\
N1-C1 & $1.162(4)$ & C5B-C6B & $1.53(2)$ \\
N2-C2 & $1.182(3)$ & C6B-C7B & $1.514(8)$ \\
N3-C3 & $1.178(4)$ & & \\
N2-Sb1-N3 & $86.5(1)$ & C7A-O1A-C4A & $109.2(4)$ \\
N2-Sb1-N1 & $86.36(9)$ & C7A-O1A-Sb1 & $118.8(3)$ \\
N3-Sb1-N1 & $85.9(1)$ & C4A-O1A-Sb1 & $120.6(4)$ \\
N2-Sb1-O1B & $81.3(2)$ & O1A-C4A-C5A & $102.7(4)$ \\
N3-Sb1-O1B & $86.0(3)$ & C4A-C5A-C6A & $100.0(6)$ \\
N1-Sb1-O1B & $165.6(3)$ & C7A-C6A-C5A & $102.0(5)$ \\
N2-Sb1-O1A & $76.6(2)$ & O1A-C7A-C6A & $105.1(4)$ \\
N3-Sb1-O1A & $77.0(2)$ & C7B-O1B-C4B & $108.6(6)$ \\
N1-Sb1-O1A & $156.4(2)$ & C7B-O1B-Sb1 & $120.8(6)$ \\
C1-N1-Sb1 & $145.3(3)$ & C4B-O1B-Sb1 & $130.0(7)$ \\
C2-N2-Sb1 & $138.2(2)$ & O1B-C4B-C5B & $104.0(7)$ \\
C3-N3-Sb1 & $146.2(2)$ & C4B-C5B-C6B & $101.8(9)$ \\
N1-C1-S1 & $178.3(3)$ & C7B-C6B-C5B & $99.4(8)$ \\
N2-C2-S2 & $176.7(2)$ & O1B-C7B-C6B & $105.3(6)$ \\
N3-C3-S3 & $176.7(3)$ & & \\
\hline
\end{tabular}


Figure S5. ORTEP representation of $\mathrm{Sb}(\mathrm{NCS})_{3} \cdot \operatorname{thf} \mathbf{3 b}$. Thermal ellipsoids with $50 \%$ probability at $123 \mathrm{~K}, \mathrm{H}$ atoms are omitted for clarity.
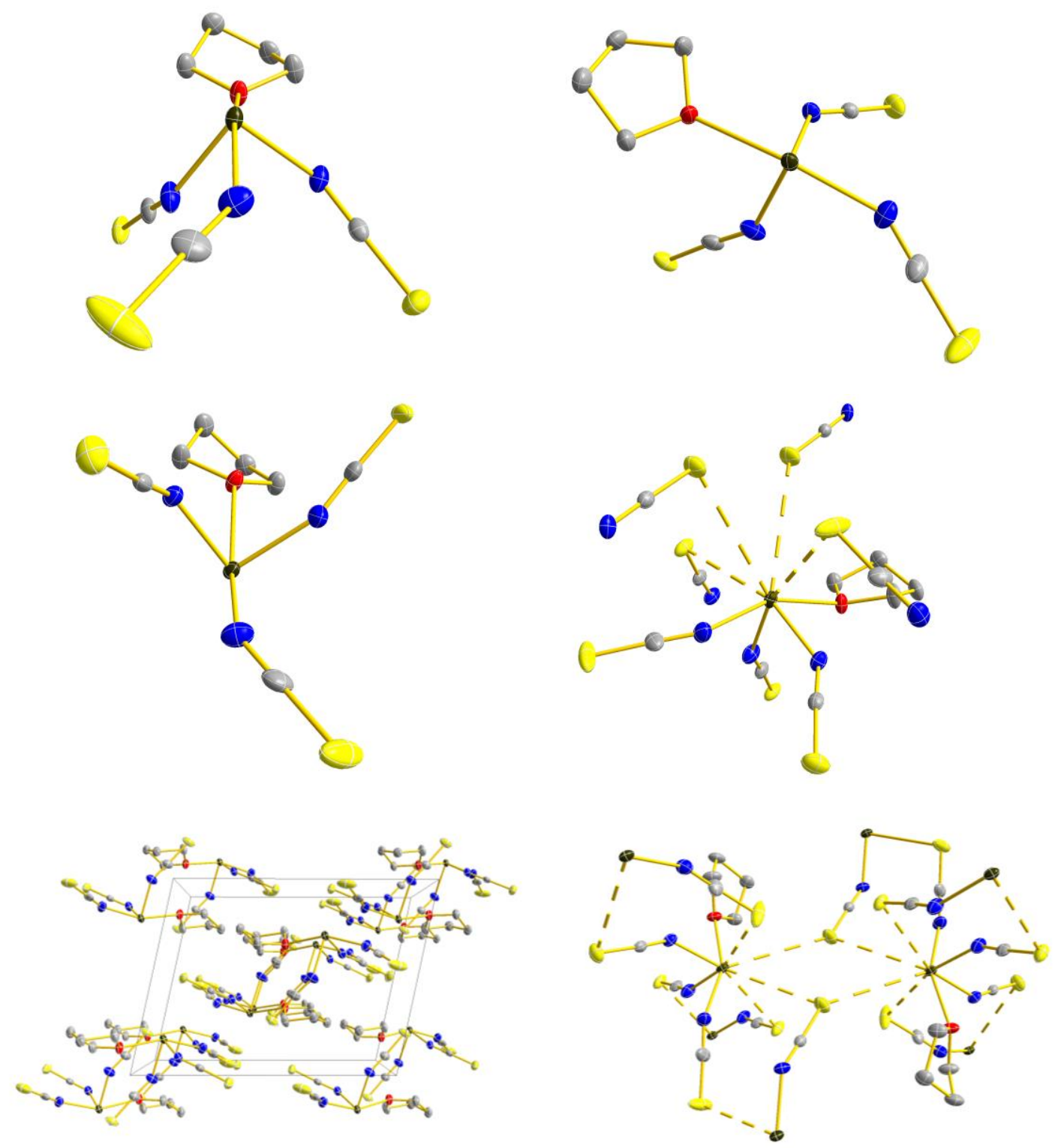
Scheme S6. Numbering scheme of $\mathrm{Bi}(\mathrm{NCS})_{3} \cdot \operatorname{thf} \mathbf{4}$.

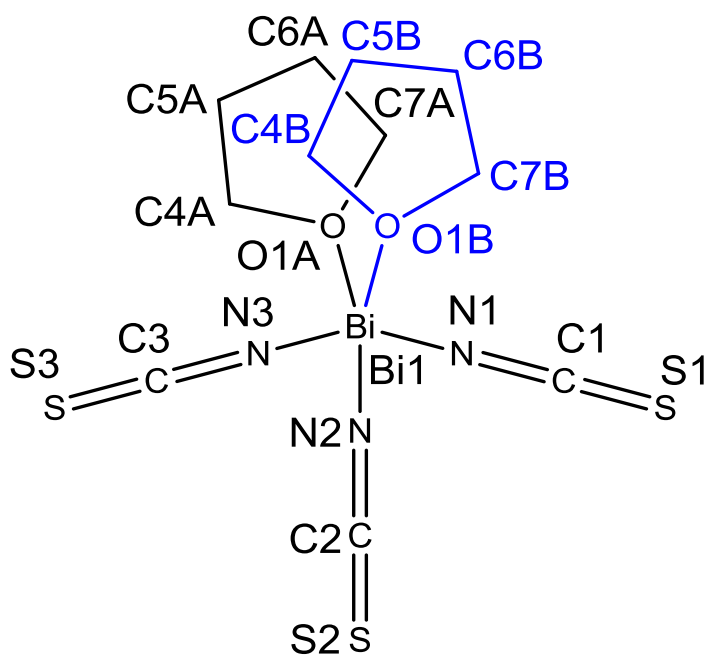

Table S9. Selected bond lengths $(\AA)$, distances $(\AA)$ and angles $\left({ }^{\circ}\right)$ of $\operatorname{Bi}(\mathrm{NCS})_{3} \cdot \operatorname{thf} 4$.

\begin{tabular}{|c|c|c|c|}
\hline $\mathrm{Bi} 1-\mathrm{N} 2$ & $2.412(5)$ & $\mathrm{S} 1-\mathrm{Bi} 1^{\mathrm{i}}$ & $2.808(2)$ \\
\hline $\mathrm{Bi} 1-\mathrm{N} 1$ & $2.485(5)$ & S2-Bitiii & $2.847(2)$ \\
\hline $\mathrm{Bi1}-\mathrm{O} 1 \mathrm{~A}$ & $2.555(7)$ & S3-Bi1ii & $2.751(2)$ \\
\hline $\mathrm{Bi1}-\mathrm{N} 3$ & $2.617(5)$ & O1A-C4A & $1.45(2)$ \\
\hline $\mathrm{Bi1-O1B}$ & $2.65(2)$ & $\mathrm{O} 1 \mathrm{~A}-\mathrm{C} 7 \mathrm{~A}$ & $1.46(2)$ \\
\hline $\mathrm{Bi} 1-\mathrm{S}^{\mathrm{i}}$ & $2.751(2)$ & $\mathrm{C} 4 \mathrm{~A}-\mathrm{C} 5 \mathrm{~A}$ & $1.50(2)$ \\
\hline $\mathrm{Bi} 1-\mathrm{S} 1^{\mathrm{ii}}$ & $2.808(2)$ & $\mathrm{C} 5 \mathrm{~A}-\mathrm{C} 6 \mathrm{~A}$ & $1.50(2)$ \\
\hline $\mathrm{Bi} 1-\mathrm{S} 2 \mathrm{iii}$ & $2.847(2)$ & $\mathrm{C} 6 \mathrm{~A}-\mathrm{C} 7 \mathrm{~A}$ & $1.51(1)$ \\
\hline $\mathrm{N} 1-\mathrm{C} 1$ & $1.151(7)$ & O1B-C4B & $1.45(2)$ \\
\hline $\mathrm{N} 2-\mathrm{C} 2$ & $1.158(7)$ & O1B-C7B & $1.46(2)$ \\
\hline N3-C3 & $1.153(7)$ & C4B-C5B & $1.50(2)$ \\
\hline $\mathrm{S} 1-\mathrm{C} 1$ & $1.658(6)$ & C5B-C6B & $1.50(2)$ \\
\hline S2-C2 & $1.643(5)$ & C6B-C7B & $1.50(2)$ \\
\hline S3-C3 & $1.663(5)$ & & \\
\hline N2-Bi1-N1 & $147.4(2)$ & S3-Bi1-S2iii & $91.35(4)$ \\
\hline N2-Bi1-O1A & $73.9(5)$ & $\mathrm{S} 1^{\mathrm{ii}-\mathrm{Bi} 1-\mathrm{S} 2 \mathrm{iii}}$ & $164.43(4)$ \\
\hline N1-Bi1-O1A & $127.7(3)$ & $\mathrm{C} 1-\mathrm{N} 1-\mathrm{Bi1}$ & $161.8(4)$ \\
\hline N2-Bi1-N3 & $139.3(2)$ & C2-N2-Bi1 & $159.0(4)$ \\
\hline N1-Bi1-N3 & $73.2(2)$ & C3-N3-Bi1 & $161.3(4)$ \\
\hline O1A-Bi1-N3 & $74.4(6)$ & $\mathrm{C} 1-\mathrm{S} 1-\mathrm{Bi} 1^{\mathrm{i}}$ & $101.6(2)$ \\
\hline
\end{tabular}




\begin{tabular}{|c|c|c|c|}
\hline N2-Bi1-O1B & $73(2)$ & $\mathrm{C} 2-\mathrm{S} 2-\mathrm{Bi} 1^{\mathrm{iii}}$ & $98.4(2)$ \\
\hline N1-Bi1-O1B & $128.7(9)$ & C3-S3-Bi1 ${ }^{i i}$ & $99.0(2)$ \\
\hline N3-Bi1-O1B & $74(2)$ & $\mathrm{N} 1-\mathrm{C} 1-\mathrm{S} 1$ & $178.0(5)$ \\
\hline N2-Bi1-S3i & $72.7(2)$ & N2-C2-S2 & $177.8(5)$ \\
\hline N1-Bi1-S3i & $81.8(2)$ & N3-C3-S3 & $179.0(5)$ \\
\hline O1A-Bi1-S3i & $146.5(5)$ & C4A-01A-C7A & $105.2(9)$ \\
\hline N3-Bi1-S3i & $136.3(2)$ & C4A-01A-Bi1 & $126.7(6)$ \\
\hline O1B-Bi1-S3i & $146(2)$ & C7A-01A-Bi1 & $122.9(6)$ \\
\hline $\mathrm{N} 2-\mathrm{Bi} 1-\mathrm{S} 1^{\mathrm{ii}}$ & $83.8(2)$ & O1A-C4A-C5A & $104.2(8)$ \\
\hline $\mathrm{N} 1-\mathrm{Bi} 1-\mathrm{S} 1^{\mathrm{ii}}$ & $109.1(2)$ & C6A-C5A-C4A & $105.7(6)$ \\
\hline O1A-Bi1-S1ii & $104.5(4)$ & C5A-C6A-C7A & $105.4(7)$ \\
\hline N3-Bi1-S1ii & $80.3(2)$ & O1A-C7A-C6A & $105.3(8)$ \\
\hline O1B-Bi1-S1ii & $103(2)$ & C4B-01B-C7B & $105(2)$ \\
\hline S3i-Bi1-S1 ${ }^{i i}$ & $74.77(4)$ & C4B-O1B-Bi1 & $136(2)$ \\
\hline N2-Bi1-S2iii & $85.4(2)$ & C7B-01B-Bi1 & $114(2)$ \\
\hline N1-Bi1-S2iii & $75.1(2)$ & O1B-C4B-C5B & $105(2)$ \\
\hline O1A-Bi1-S2iii & $83.1(5)$ & C6B-C5B-C4B & $106(1)$ \\
\hline N3-Bi1-S2 $2^{\mathrm{iii}}$ & $115.1(2)$ & C5B-C6B-C7B & $105(1)$ \\
\hline O1B-Bi1-S2iii & $84(2)$ & O1B-C7B-C6B & $105(2)$ \\
\hline
\end{tabular}

Symmetry codes: (i) $-\mathrm{x}+1, \mathrm{y}+1 / 2,-\mathrm{z}+3 / 2$; (ii) $-\mathrm{x}+1, \mathrm{y}-1 / 2,-\mathrm{z}+3 / 2$; (iii) $-\mathrm{x}+1,-\mathrm{y}+1,-\mathrm{z}+1$.

Figure S6. ORTEP representation of $\mathrm{Bi}(\mathrm{NCS})_{3} \cdot$ thf 4 . Thermal ellipsoids with $50 \%$ probability at $123 \mathrm{~K}, \mathrm{H}$ atoms are omitted for clarity.
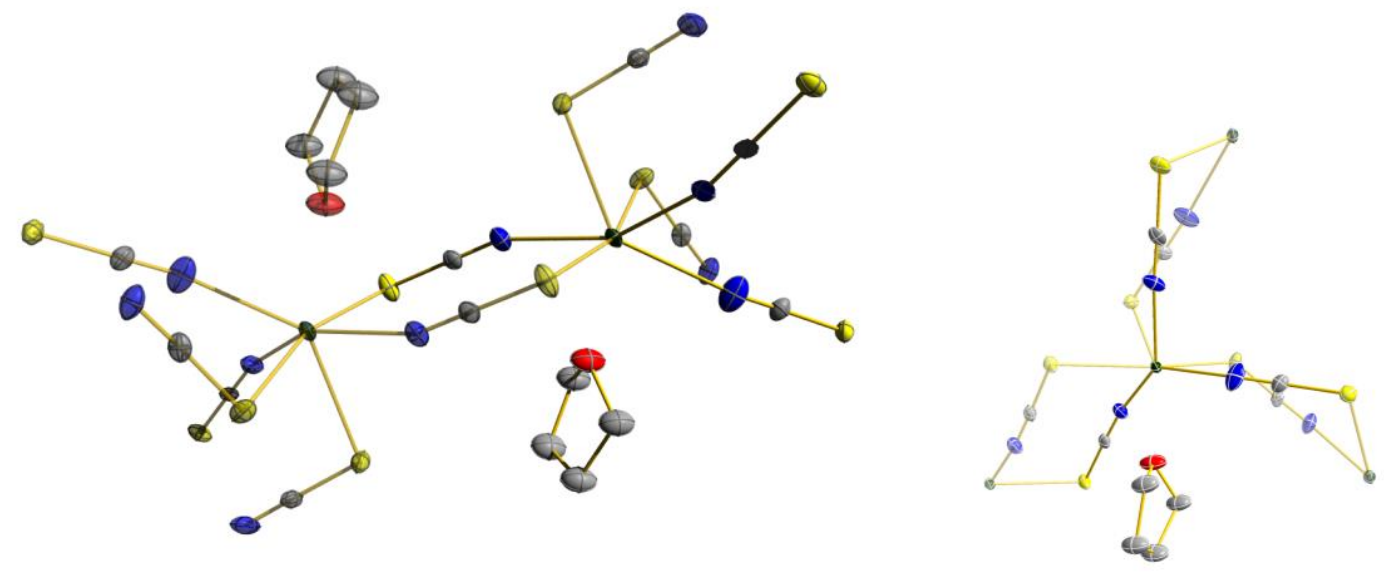
Scheme S7. Numbering scheme of the anion of $[\mathrm{PPN}]_{2}\left[\mathrm{Sb}(\mathrm{NCS})_{5}\right] \mathbf{5}$.

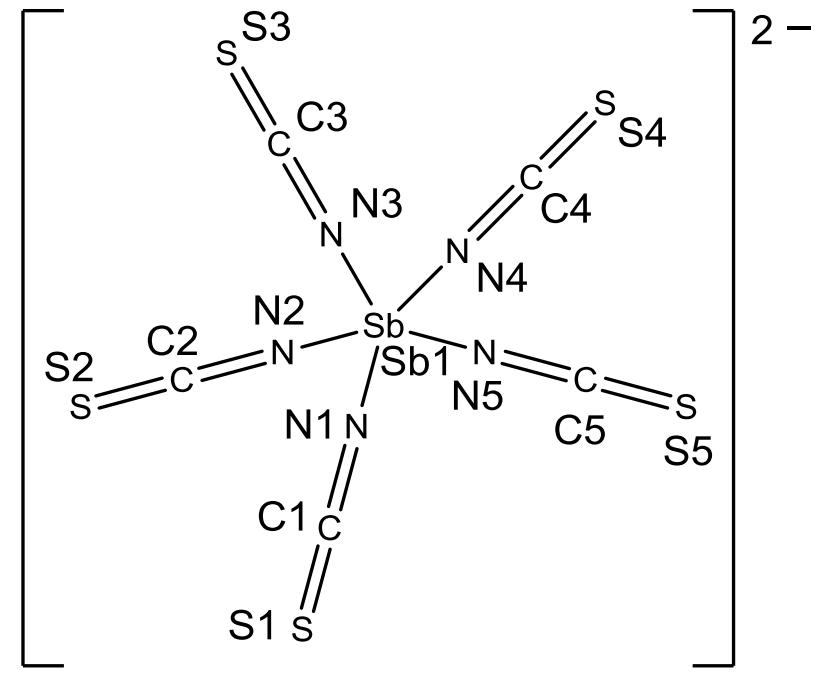

Table S10. Selected bond lengths $(\AA)$, distances $(\AA)$ and angles $\left(^{\circ}\right)$ of $[\mathrm{PPN}]_{2}\left[\mathrm{Sb}(\mathrm{NCS})_{5}\right] 5$.

\begin{tabular}{|c|c|c|c|}
\hline Sb1-N3 & $2.045(3)$ & S4-C4 & $1.606(4)$ \\
\hline Sb1-N4 & 2.206 (3) & S5-C5 & $1.617(4)$ \\
\hline Sb1-N2 & $2.254(4)$ & $\mathrm{N} 1-\mathrm{C} 1$ & $1.154(5)$ \\
\hline Sb1-N5 & $2.284(3)$ & N2-C2 & $1.158(5)$ \\
\hline Sb1-N1 & $2.322(4)$ & N3-C3 & $1.171(4)$ \\
\hline S1-C1 & $1.620(4)$ & N4-C4 & $1.163(5)$ \\
\hline S2-C2 & $1.609(4)$ & N5-C5 & $1.173(5)$ \\
\hline S3-C3 & $1.591(4)$ & & \\
\hline N3-Sb1-N4 & $83.0(2)$ & $\mathrm{C} 1-\mathrm{N} 1-\mathrm{Sb} 1$ & 149.9 (3) \\
\hline N3-Sb1-N2 & $81.3(2)$ & C2-N2-Sb1 & $169.1(3)$ \\
\hline N4-Sb1-N2 & $88.4(2)$ & C3-N3-Sb1 & $161.2(3)$ \\
\hline N3-Sb1-N5 & $82.3(2)$ & C4-N4-Sb1 & 140.1 (3) \\
\hline N4-Sb1-N5 & $85.5(2)$ & C5-N5-Sb1 & $149.8(3)$ \\
\hline N2-Sb1-N5 & $163.2(2)$ & $\mathrm{N} 1-\mathrm{C} 1-\mathrm{S} 1$ & $178.9(4)$ \\
\hline N3-Sb1-N1 & $79.5(2)$ & N2-C2-S2 & $177.5(4)$ \\
\hline N4-Sb1-N1 & $162.4(2)$ & N3-C3-S3 & $178.5(4)$ \\
\hline N2-Sb1-N1 & $90.7(2)$ & N4-C4-S4 & $177.4(4)$ \\
\hline N5-Sb1-N1 & $90.3(2)$ & N5-C5-S5 & $178.2(3)$ \\
\hline
\end{tabular}


Figure S7. ORTEP representation of the anion of $[\mathrm{PPN}]_{2}\left[\mathrm{Sb}(\mathrm{NCS})_{5}\right]$ 5. Thermal ellipsoids with $50 \%$ probability at $123 \mathrm{~K}, \mathrm{H}$ atoms are omitted for clarity.

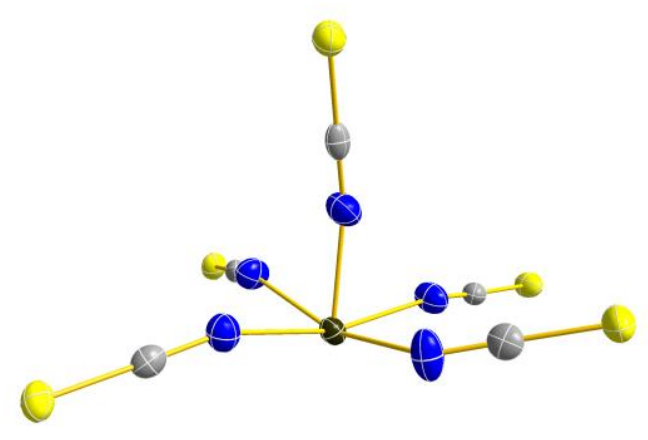


Scheme S8. Numbering scheme of $[\mathrm{PPN}]_{2}\left[\mathrm{Bi}(\mathrm{NCS})_{3}(\mathrm{SCN})_{2}(\right.$ thf $\left.)\right] 6$.

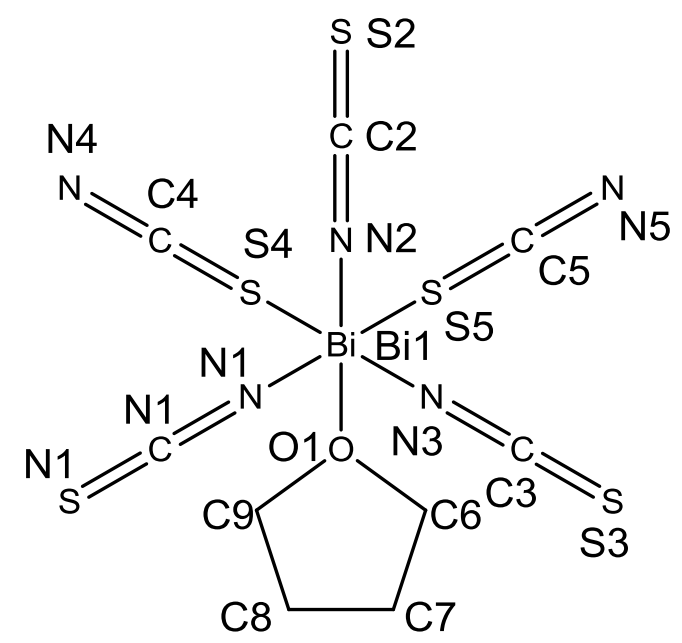

Table S11. Selected bond lengths $(\AA)$, distances $(\AA)$ and angles $\left({ }^{\circ}\right)$ of $[\mathrm{PPN}]_{2}\left[\mathrm{Bi}(\mathrm{NCS})_{3}(\mathrm{SCN})_{2}(\mathrm{thf})\right] 6$.

\begin{tabular}{|c|c|c|c|}
\hline $\mathrm{Bi} 1-\mathrm{N} 2$ & $2.282(6)$ & $\mathrm{N} 1-\mathrm{C} 1$ & $1.157(9)$ \\
\hline Bi1-N3 & $2.361(6)$ & N2-C2 & $1.036(9)$ \\
\hline $\mathrm{Bi} 1-\mathrm{N} 1$ & $2.449(6)$ & N3-C3 & $1.160(9)$ \\
\hline $\mathrm{Bi} 1-\mathrm{O} 1$ & $2.648(5)$ & N4-C4 & $1.16(2)$ \\
\hline Bi1-S4 & $2.739(2)$ & N5-C5 & $1.17(1)$ \\
\hline Bi1-S5 & $2.836(2)$ & O1-C9 & $1.418(8)$ \\
\hline $\mathrm{S} 1-\mathrm{C} 1$ & 1.617 (8) & O1-C6 & $1.435(8)$ \\
\hline S2-C2 & 1.647 (9) & $\mathrm{C} 6-\mathrm{C} 7$ & $1.523(9)$ \\
\hline S3-C3 & $1.618(7)$ & C7-C8 & $1.557(9)$ \\
\hline S4-C4 & $1.64(2)$ & C8-C9 & $1.51(1)$ \\
\hline S5-C5 & $1.648(8)$ & & \\
\hline N2-Bi1-N3 & $85.1(2)$ & C5-S5-Bi1 & $94.0(2)$ \\
\hline N2-Bi1-N1 & $84.3(2)$ & $\mathrm{C} 1-\mathrm{N} 1-\mathrm{Bi1}$ & 173.8 (5) \\
\hline N3-Bi1-N1 & $169.3(2)$ & C2-N2-Bi1 & 123.9 \\
\hline N2-Bi1-O1 & $160.5(2)$ & C3-N3-Bi1 & 159.5 (5) \\
\hline N3-Bi1-O1 & $78.5(2)$ & $\mathrm{N} 1-\mathrm{C} 1-\mathrm{S} 1$ & $179.7(8)$ \\
\hline N1-Bi1-O1 & $111.6(2)$ & N2-C2-S2 & $174.2(7)$ \\
\hline N2-Bi1-S4 & $90.4(2)$ & N3-C3-S3 & $177.8(6)$ \\
\hline N3-Bi1-S4 & $89.3(2)$ & N4-C4-S4 & $176.1(8)$ \\
\hline N1-Bi1-S4 & $89.2(2)$ & N5-C5-S5 & $179.3(7)$ \\
\hline
\end{tabular}




\begin{tabular}{llll} 
O1-Bi1-S4 & $79.1(2)$ & C9-O1-C6 & $109.7(5)$ \\
N2-Bi1-S5 & $88.3(2)$ & C9-O1-Bi1 & $117.6(4)$ \\
N3-Bi1-S5 & $89.2(2)$ & C6-O1-Bi1 & $129.7(4)$ \\
N1-Bi1-S5 & $92.1(2)$ & O1-C6-C7 & $107.4(5)$ \\
O1-Bi1-S5 & $101.8(2)$ & C6-C7-C8 & $103.0(6)$ \\
S4-Bi1-S5 & $178.1(1)$ & C9-C8-C7 & $101.9(5)$ \\
C4-S4-Bi1 & $102.4(3)$ & O1-C9-C8 & $106.0(6)$ \\
\hline
\end{tabular}

Figure S8. ORTEP representation of the anion of $[\mathrm{PPN}]_{2}\left[\mathrm{Bi}(\mathrm{NCS})_{3}(\mathrm{SCN})_{2}(\right.$ thf $\left.)\right] 6$. Thermal ellipsoids with $50 \%$ probability at $123 \mathrm{~K}, \mathrm{H}$ atoms are omitted for clarity.
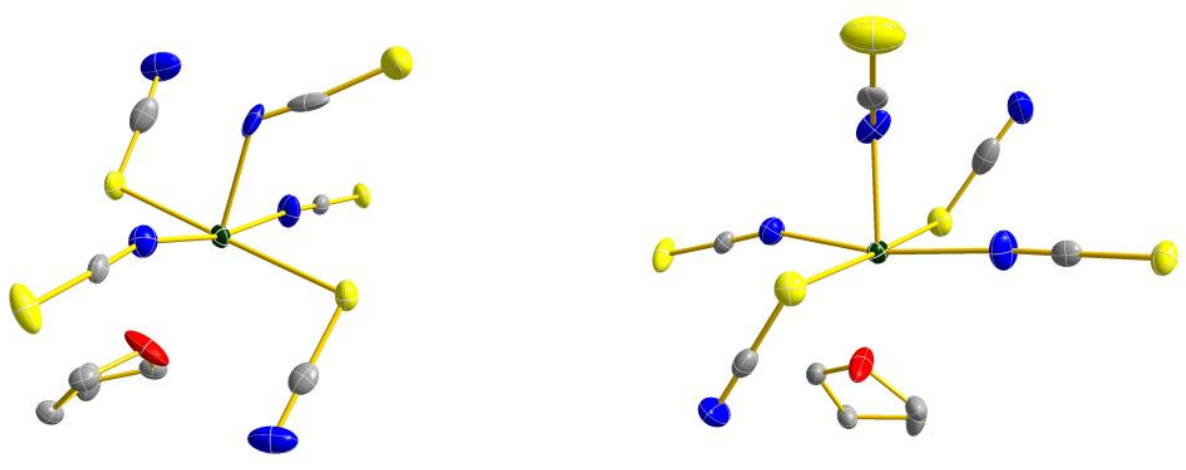


\section{Syntheses of Compounds}

$[P P N] S C N(1)$

$$
[\mathrm{PPN}] \mathrm{Cl}+\mathrm{KSCN} \longrightarrow[\mathrm{H} \mathrm{O} \longrightarrow[\mathrm{PPN}] \mathrm{SCN}+\mathrm{KCl}
$$

The $[\mathrm{PPN}] \mathrm{Cl}(12.8 \mathrm{~g}, 22.3 \mathrm{mmol})$ is dissolved in hot water $\left(80^{\circ} \mathrm{C}, 100 \mathrm{~mL}\right) . \mathrm{KSCN}(4.3 \mathrm{~g}$, $44.2 \mathrm{mmol})$ is dissolved in water $(20 \mathrm{~mL})$, too. Subsequently, both solutions will be united. The white residue is filtered and washed with water $(3 \times 20 \mathrm{~mL})$. After drying in air the product is crystallized from acetone $(75 \mathrm{~mL})$. The product [PPN]SCN is dried in vacuo and isolated with a yield of $11.36 \mathrm{~g}(85.3 \%)$.

Mp. $193{ }^{\circ} \mathrm{C}$. CHNS calc. (found) in \%: C 74.48 (74.16); H 5.07 (5.19); N 4.69 (4.65); S 5.37 (5.18). ${ }^{1}$ H NMR (300 MHz, c $\left.d_{3} \mathrm{cn}\right) \delta 7.72-7.41$ (m). ${ }^{13} \mathbf{C}$ NMR (75 MHz, c $\left.d_{3} \mathrm{cn}\right) \delta 134.6$ (s, $\left.C_{\text {para }}\right), 133.5-133.1$ (m, $\left.C_{\text {metha }}\right), 130.6-130.2$ (m, $\left.C_{\text {ortho }}\right), 128.2\left(\mathrm{dd},{ }^{1} J\left[{ }^{13} C,{ }^{31} \mathrm{P}\right]=108 \mathrm{~Hz}\right.$, $\left.{ }^{3} J\left[{ }^{13} \mathrm{C},{ }^{31} \mathrm{P}\right]=2 \mathrm{~Hz}, C_{\mathrm{ipso}}\right) .{ }^{31} \mathbf{P}$ NMR $\left(121 \mathrm{MHz}, \mathrm{c} d_{3} \mathrm{cn}\right) \delta 20.8$. IR (ATR, $25^{\circ} \mathrm{C}, 32 \mathrm{scans}, \mathrm{cm}^{-}$ 1): 3059 (w), 3012 (w), 2052 (m), 1587 (w) 1576 (w), 1481 (w), 1435 (m), 1410 (w), 1319 (m), 1304 (s), 1286 (m), 1273 (s), 1178 (m), 1161 (w), 1111 (s), 1076 (m), 1026(w), 997 (m), 974(w), $924(\mathrm{w}), 795(\mathrm{w}), 764(\mathrm{~m}), 741(\mathrm{~m}), 719(\mathrm{~s}), 702(\mathrm{~s}), 687$ (s), $663(\mathrm{~m}), 615(\mathrm{w}), 546(\mathrm{~s}), 528$ (vs). Raman (633 nm, D00, x10, 20s, 20 acc., cm ${ }^{-1}$ ): 3172 (0), 3148 (0), 3146 (0), 3143 (0), 3089 (0), 3063 (3), 3057 (3), 3013 (0), 2989 (0), 2052 (5), 1587 (5), 1574 (1), 1193 (0), 1185 (0), 1177 (1), 1162 (0), 1159 (0), 1152 (1), 1115 (2), 1107 (3), 1025 (5), 998 (10), 728 (1), 660 (3), 613 (2), 542 (0), 485 (0), 361 (0), 278 (0), 264 (1), 249 (1), 232 (3), 222 (1).

$[\mathrm{PPN}]\left[\mathrm{H}(\mathrm{NCS})_{2}\right](2)$ $[\mathrm{PPN}] \mathrm{SCN}+\mathrm{Me}_{3} \mathrm{Si}-\mathrm{NCS}+\mathrm{MeOH} \stackrel{\mathrm{CH}_{3} \mathrm{CN}}{\longrightarrow}[\mathrm{PPN}]\left[\mathrm{H}(\mathrm{NCS})_{2}\right]+\mathrm{Me}_{3} \mathrm{Si}-\mathrm{OMe}$ [PPN]SCN (1.475 g, $2.47 \mathrm{mmol})$ and trimethylsilylisothiocyanate $(0.70 \mathrm{~mL}, 0.648 \mathrm{~g}$, $4.94 \mathrm{mmol})$ are dissolved in acetonitril $(2 \mathrm{~mL})$. Subsequently, methanol $(0.1 \mathrm{~mL}, 0.079 \mathrm{~g}$, $2.47 \mathrm{mmol}$ ) is added and stirred for 12 hours. The solvent and excess reactants are removed in vacuo. The product has a yield of $0.956 \mathrm{~g}(98.8 \%)$.

Mp. $129{ }^{\circ}$ C. CHNS calc. (found) in \%: C 69.60 (69.65), H 4.76 (4.96), N 6.49 (6.52), S 9.78 (9.80). ${ }^{1} \mathbf{H}$ NMR $\left(300 \mathrm{MHz}, \mathrm{c} d_{3} \mathrm{cn}\right) \delta 7.77-7.33(\mathrm{~m}) .{ }^{13} \mathbf{C}$ NMR $\left(75 \mathrm{MHz}, \mathrm{c} d_{3} \mathrm{cn}\right) \delta$ 134.6 (s, $\left.C_{\text {para }}\right), 133.5$ - 133.0 (m, $\left.C_{\text {metha }}\right), 130.6$ - 130.1 (m, $\left.C_{\text {ortho }}\right), 128.2$ (dd, ${ }^{1} J\left[{ }^{13} C,{ }^{31} P\right]=$ $\left.108 \mathrm{~Hz},{ }^{3} J\left[{ }^{13} C,{ }^{31} P\right]=2 \mathrm{~Hz}, C_{\text {ipso }}\right) .{ }^{31} \mathbf{P}$ NMR $\left(121 \mathrm{MHz}, \mathrm{c} d_{3} \mathrm{cn}\right) \delta 20.8$. IR (ATR, $25^{\circ} \mathrm{C}, 32$ scans, $\mathrm{cm}^{-1}$ ): 3076 (w), 3055 (w), 2048 (s, br), 1589 (w), 1481 (w), 1435 (m), 1296 (s), 1279 
(s), 1265 (s), 1184 (m), 1169 (m), $1113(\mathrm{~s}), 1070$ (m), 1024 (m), 997 (s), 972 (m), 908 (s), 847 (m), 797 (w), 744 (m), 723 (s), 687 (vs), 663 (s), 619 (s), 608 (s), 571 (s), 542 (vs). Raman (633 nm, D00, x10, 20s, 20 acc., cm ${ }^{-1}$ ): 3056 (4), 2051 (1), 1588(4), 1573 (1), 1182 (1), 1160 (1), 1109 (3), 1025 (4), 1000 (10), 662 (3), 613 (2), 357 (1), 282 (0), 264 (2), 256 (1), 246 (1), 232 (2), 227 (2).

$\mathrm{Sb}(\mathrm{NCS})_{3} / \mathrm{Sb}(\mathrm{NCS})_{3} \cdot \operatorname{thf}(\mathbf{3})$

$$
\mathrm{SbF}_{3}+3 \mathrm{Me}_{3} \mathrm{Si}-\mathrm{NCS} \longrightarrow \mathrm{Sb}(\mathrm{NCS})_{3}+3 \mathrm{Me}_{3} \mathrm{SiF}
$$

$\mathrm{SbF}_{3}(0.5 \mathrm{~g}, 2.8 \mathrm{mmol})$ is dissolved in thf $(10 \mathrm{~mL})$. Subsequently, trimethylsilylisothiocyanate (3.5 mL, $3.305 \mathrm{~g}, 25.2 \mathrm{mmol}$ ) is added and stirred for $16 \mathrm{~h}$ at ambient temperature. After removing the solvent in vacuo the product $\mathbf{S b}(\mathbf{N C S})_{3} \cdot$ thf is isolated with a yield of $0.870 \mathrm{~g}$ $(84.4 \%)$.

Sb(NCS)3 thf (366.9 g/mol) MP. $130{ }^{\circ} \mathrm{C}$ (decomposition). CHNS calc. (found) in \%: C: 22.41 (22.84), H: 2.15 (2.19), N 11.34 (11.42), S: 26.66 (26.13). IR (ATR, 25 ${ }^{\circ} \mathrm{C}, 128$ scans, cm ${ }^{-1}$ ): 2980 (m), 2955 (m), 2930 (m), 2883 (m), 2490 (m), 2328 (w), 1932 (vs, br), 1603 (s), 1493 (s), 1479 (s), 1454 (s), 1446 (s), 1379 (s), 1362 (s), 1294 (s), 1254 (s), 1173 (s), 1136 (s), 1032 (s), 1013 (s), 953 (s), 916 (s), 885 (s), 827 (s), 766 (s), 663 (s), 582 (s), 528 (s). Raman (633 nm, D00, x10, 30s, 30acc, $\mathrm{cm}^{-1}$ ): 2982 (0), $2937(0), 2892$ (0), 2122 (2), 2083 (4), 2060 (3), 2029 (2), 2004 (2), 1974 (3), 1608 (1), 1494 (1), 1381 (3), 1337 (1), 1310 (1), 1017 (1), 940 (1), 922 (0), 889 (0), 851 (0), 823 (0), 736 (1), 662 (3), 597 (1), 544 (4), 506 (10), 491 (2), 470 (3), 447 (0), 410 (3), 376 (1), 337 (8), 317 (2), 274 (0), 254 (3), 214 (5). MS(EI); m/z (\%): 44 (15.3) $[\mathrm{CS}]^{+} ; 76$ (100), 77 (3.7), 78 (12.4) [CS $]^{+} ; 121$ (11.3), 123 (8.7) [Sb] 179 (48.6), 180 (1.2), 181 (34.3) [Sb(NCS)]+; 237 (50.0), 238 (2.0) 239 (41.0) [Sb(NCS) $]^{+} ; 295$ (44.9), 296 (2.6), 297 (42.3), 299 (4.1) [Sb(NCS) $]^{+}$.

Pure $\mathbf{S b}(\mathrm{NCS})_{3}$ was obtained after prolonged pumping or from the reaction of $\mathrm{SbF}_{3}$ in an excess of TMS-SCN. CHNS calc. (found) in \%: C: 12.17 (12.26), N 14.20 (13.10)

\section{$\operatorname{Bi}(\mathrm{NCS})_{3} \cdot \operatorname{thf}(\mathbf{4})$}

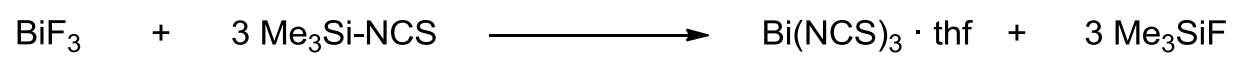


$\mathrm{BiF}_{3}(0.5 \mathrm{~g}, 1.9 \mathrm{mmol})$ is suspended in thf $(10 \mathrm{~mL})$. Subsequently, trimethylsilylisothiocyanate ( $2.4 \mathrm{~mL}, 2.2 \mathrm{~g}, 16.9 \mathrm{mmol}$ ) is added and stirred for $16 \mathrm{~h}$ at ambient temperature. The solution is filtered and the solvent removed in vacuo. The product has a yield of $0.220 \mathrm{~g}(25 \%)$.

Mp. $121{ }^{\circ} \mathrm{C}$ (decomposition). CHNS calc. (found) in \%: Bi(NCS) 3 .thf: C 18.46 (17.27), H 1.77 (1.38), N 9.23 (9.71), S 21.23 (22.57). ${ }^{1} \mathbf{H}$ NMR (250 MHz, thf- $\left.d 8\right) \delta 3.70-3.47$ (m, $\left.\mathrm{OCH}_{2}\right), 1.85-1.62\left(\mathrm{~m}, \mathrm{CH}_{2}\right), 0.30-0.23(\mathrm{~m}), 0.20-0.10(\mathrm{~m}), 0.05--0.02(\mathrm{~m}) .{ }^{13} \mathrm{C} \mathrm{NMR}(62$ MHz, thf- $d 8) \delta 68.4(\mathrm{~s}), 26.6(\mathrm{~s}), 2.2(\mathrm{~s}), 0.1$ (t, $J=7.6 \mathrm{~Hz}$ ). IR (ATR, $25^{\circ} \mathrm{C}, 32 \mathrm{scans}, \mathrm{cm}^{-1}$ ): 2945 (w), 2883 (w), 2083 (s, br), 1454 (s), 1365 (s), 1340 (s), 1022 (vs), 916 (s), 858 (vs), 771 (s), 731 (s), 662 (s). Raman (633 nm, D06, x10, 10s, 20acc, cm ${ }^{-1}$ ): 2986 (0), 2886 (0), 2105 (10), 2072 (2), 915 (0), 885 (0), 773 (0), 750 (1), 731 (1), 463 (1), 447 (1), 443 (1), 223 (6), 194 (3), $157(3)$.

Solvent free $\mathrm{Bi}(\mathrm{NCS})_{3}$ was obtained after prolonged pumping in vacuo: C 9.40 (9.37), H 0.0 (0.17), N 10.96 (10.70), S 25.10 (24.60).

$[\mathrm{PPN}]_{2}\left[\mathrm{Sb}(\mathrm{NCS})_{5}\right](\mathbf{5})$

$\mathrm{SbF}_{3}+2[\mathrm{PPN}] \mathrm{SCN}+3 \mathrm{Me}_{3} \mathrm{Si}-\mathrm{NCS} \longrightarrow[\mathrm{PPN}]_{2}\left[\mathrm{Sb}(\mathrm{NCS})_{5}\right]+3 \mathrm{Me}_{3} \mathrm{SiF}$

[PPN]SCN (4.4 g, $7.37 \mathrm{mmol})$ and $\mathrm{SbF}_{3}(0.4394 \mathrm{~g}, 2.46 \mathrm{mmol})$ are dissolved in acetonitrile $(20 \mathrm{~mL})$. Subsequently, trimethylsilylisothiocyanate $(4.0 \mathrm{~mL}, 3.724 \mathrm{~g}, 22.4 \mathrm{mmol})$ is added and stirred at room temperature for $16 \mathrm{~h}$. After removing the solvent the crude product is recrystallized from a thf solution $(25 \mathrm{~mL})$. The product is isolated with a yield of $1.254 \mathrm{~g}$ $(11.43 \%)$.

Mp. $141^{\circ} \mathrm{C}$. CHNS calc. (found) in \%: C 62.10 (61.53), H 4.06 (4.00), N 6.58 (6.28), S 10.77 (10.56). ${ }^{1} \mathbf{H}$ NMR $\left(300 \mathrm{MHz}, \mathrm{c} d_{3} \mathrm{cn}\right) \delta 7.72-7.42\left(\mathrm{~m}, 60 \mathrm{H}, H_{\mathrm{Ph}}\right), 3.99$ (s, br), $3.69-$ $3.60\left(\mathrm{~m}, \mathrm{OCH}_{2}\right), 1.83-1.77\left(\mathrm{~m}, \mathrm{CH}_{2}\right) .{ }^{13} \mathrm{C} \mathrm{NMR}(75 \mathrm{MHz}, \mathrm{cd} 3 \mathrm{cn}) \delta 140.1(\mathrm{SCN}), 134.6\left(C_{\text {para }}\right)$, $133.4-133.1$ (m, $C_{\text {metha }}$ ), 130.6 - 130.1 (m, $C_{\text {ortho }}$ ), $128.2\left(\mathrm{dd},{ }^{1} J\left[{ }^{13} C,{ }^{31} P\right]=108 \mathrm{~Hz},{ }^{3} J\left[{ }^{13} C,{ }^{31} P\right]\right.$ $\left.=2 \mathrm{~Hz}, C_{\mathrm{ipso}}\right) .{ }^{13} \mathrm{C} \mathrm{IG} \mathrm{NMR}\left(63 \mathrm{MHz}, \mathrm{c} d_{3} \mathrm{cn}\right) \delta 138.1(\mathrm{~s} \mathrm{br}, 5 \mathrm{C}, \mathrm{SCN}), 134.6\left(\mathrm{~s}, 12 \mathrm{C}, C_{\text {para }}\right)$,

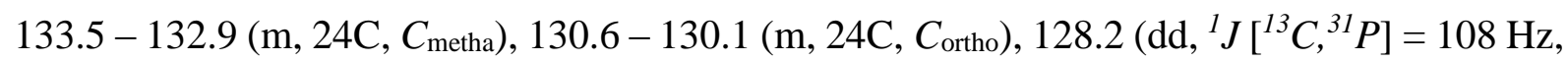
$\left.{ }^{3} J\left[{ }^{13} \mathrm{C},{ }^{31} \mathrm{P}\right]=2 \mathrm{~Hz}, 12 \mathrm{C}, C_{\mathrm{ipso}}\right) .{ }^{31} \mathbf{P}$ NMR $\left(121 \mathrm{MHz}, \mathrm{c} d_{3} \mathrm{cn}\right) \delta 20.8$. IR (ATR, $25^{\circ} \mathrm{C}, 32$ scans, $\mathrm{cm}^{-1}$ ): 3051 (w), 2073 (m), 2037 (m),2013 (s), 1988 (s), 1975 (s), 1821 (w), 1587 (m), 1576 (w), 1481 (m), 1437 (s), 1298 (s), 1281 (s), 1265 (s), 1244 (s), 1182 (s), 1161 (m), 1109 (s), 1070 (m), 1026 (m), 997 (s), 984 (m), 953 (m), 928 (m), 922 (m), 887 (m), 856 (m), 847 (m), 825 (m), 804 (m), 762 (m), 744 (s), 721 (s), 689 (vs), 665 (s), 617 (m), 546 (vs), 530 (s). 
Raman (633 nm, D03, x10, 10s, 20 acc, cm cm $^{-1} 3058$ (5), 2076 (1), 2068(1), 2042 (2), 2026 (5), 1993 (1), 1589 (5), 1576 (1), 1440 (1), 1185 (1), 1164 (2), 1111 (3), 1030 (5), 1003 (10), 808 (1), 725 (0), 666 (4), 616 (3), 486 (3), 477 (1), 335 (1), 268 (2), 243 (3), 237 (3), 229 (1).

$[\mathrm{PPN}]_{2}\left[\mathrm{Bi}(\mathrm{NCS})_{3}(\mathrm{SCN})_{2}(\right.$ thf $\left.)\right](\mathbf{6})$

$$
\mathrm{BiF}_{3}+2[\mathrm{PPN}] \mathrm{SCN}+3 \mathrm{Me}_{3} \mathrm{Si}-\mathrm{NCS} \longrightarrow[\mathrm{PPN}]_{2}\left[\mathrm{Bi}(\mathrm{NCS})_{5}(\mathrm{thf})\right]+3 \mathrm{Me}_{3} \mathrm{SiF}
$$

[PPN]SCN $(1.683 \mathrm{~g}, 2.82 \mathrm{mmol})$ and $\mathrm{BiF}_{3}(0.25 \mathrm{~g}, 0.94 \mathrm{mmol})$ are stirred in acetonitrile $(10 \mathrm{~mL})$. Subsequently, trimethylsilylisothiocyanate $(1.5 \mathrm{~mL}, 1.4 \mathrm{~g}, 8.46 \mathrm{mmol})$ is added and stirred at room temperature for $16 \mathrm{~h}$. After removing the solvent the crude product is recrystallized from a thf solution $(10 \mathrm{~mL})$. The product is isolated with a yield of $1.243 \mathrm{~g}$ $(26.93 \%)$.

Mp. $114{ }^{\circ}$ C. CHNS calc. (found) in \%: C 59.01 (59.34), H 4.16 (4.19), N 5.95 (5.65), S 9.72 (9.47). ${ }^{1} \mathbf{H}$ NMR (300 MHz, cd $\left.d_{3} \mathrm{cn}\right) \delta 7.85-7.33\left(\mathrm{~m}, 60 \mathrm{H}, H_{\mathrm{Ph}}\right), 3.73-3.55(\mathrm{~m}, 4 \mathrm{H}$, $\left.\mathrm{OCH}_{2}\right), 1.86-1.74\left(\mathrm{~m}, 4 \mathrm{H}, \mathrm{CH}_{2}\right) .{ }^{13} \mathrm{C}$ NMR $\left(75 \mathrm{MHz}, \mathrm{c} d_{3} \mathrm{cn}\right) \delta 134.6$ (s, $\left.C_{\mathrm{para}}\right), 133.5-133.1$ (m, $C_{\text {metha }}$ ), 130.6 - 130.2 (m, $C_{\text {ortho }}$ ), $128.2\left(\mathrm{dd},{ }^{1} J\left[{ }^{13} C,{ }^{31} \mathrm{P}\right]=108 \mathrm{~Hz},{ }^{3} J\left[{ }^{13} C,{ }^{31} \mathrm{P}\right]=2 \mathrm{~Hz}, C_{\mathrm{ipso}}\right.$ ), $68.3\left(\mathrm{~s}, \mathrm{OCH}_{2}\right), 26.2\left(\mathrm{~s}, \mathrm{CH}_{2}\right) .{ }^{31} \mathbf{P}$ NMR $\left(121 \mathrm{MHz}, \mathrm{c} d_{3} \mathrm{cn}\right) \delta 20.8$. IR (ATR, $25^{\circ} \mathrm{C}, 32$ scans, $\left.\mathrm{cm}^{-1}\right)$ : 3057(w), 3045(w), 2989(w), 2114(w), 2102(m), 2033(s), 1975(m), 1944(s), 1824(w), 1747(w), 1587(w), 1481(m), 1437(s), 1292(m), 1279(m), 1248(s), 1180(m), 1111(s), 1070(m), 1036(m), 1024(m), 997(s), 951(w), 937(m), 924(m), 876(m), 851(m), 804(m), 760(m), 744(s),

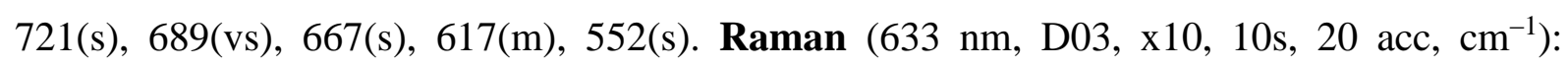
3173(0), 3150 (0), 3059 (5), 2955 (0), 2116 (5), 2054 (1), 1980 (1), 1950 (1), 1588 (5), 1575 (1), 1441 (1), 1188 (1), 1181 (1), 1165 (1), 1158 (1), 1111 (4), 1027 (4), 1002 (10), 710 (1), 668 (4)1 617 (2), 551 (0), 545 (0), 534 (1), 508 (1), 474 (1), 372 (1), 324 (5), 298 (1), 286 (1), 268 (3), 249 (5), 236 (7), 210 (10), 111 (2). 


\section{Additional Analytical Data}

[PPN]SCN (1)

TGA/DSC

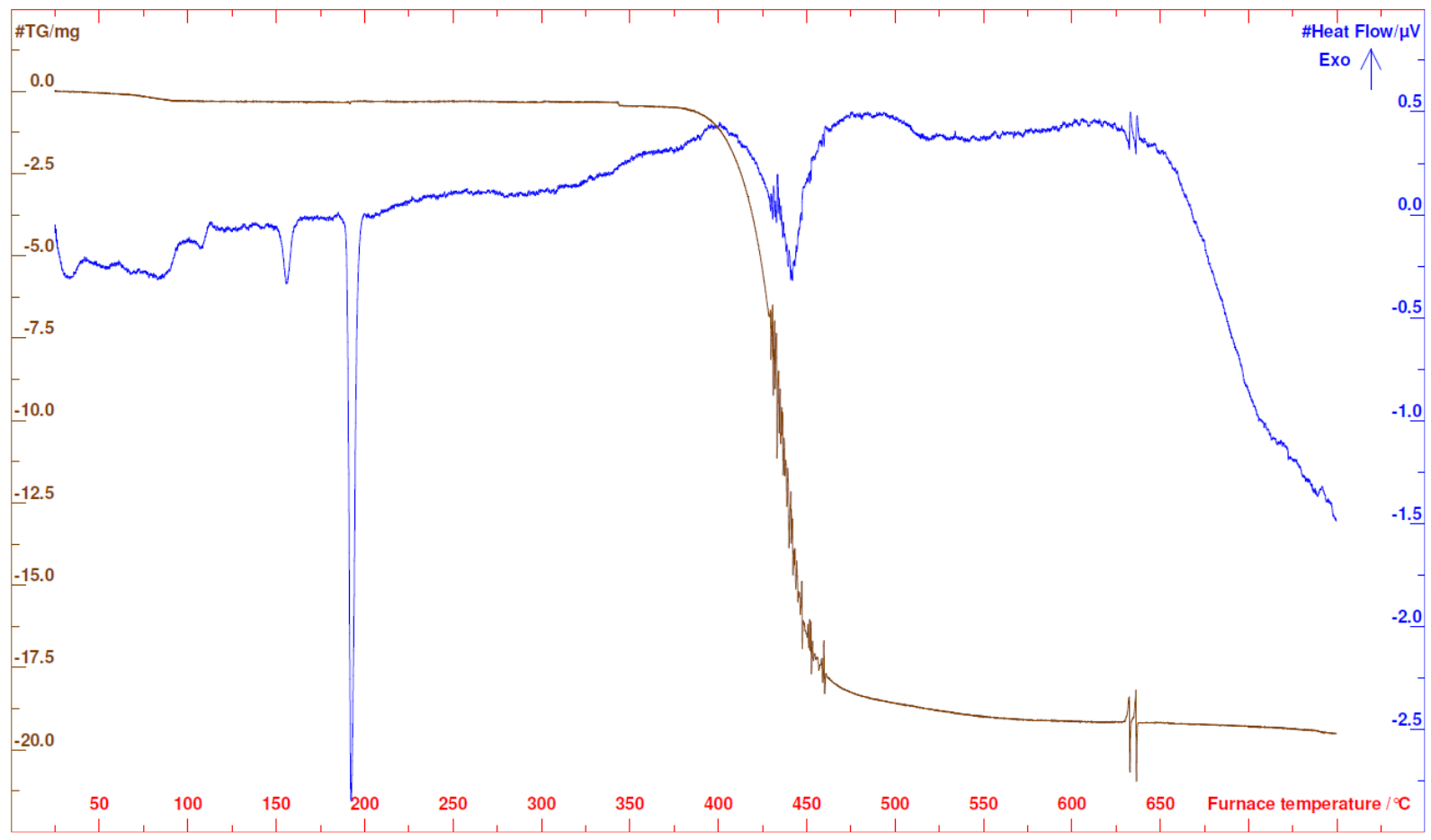

IR

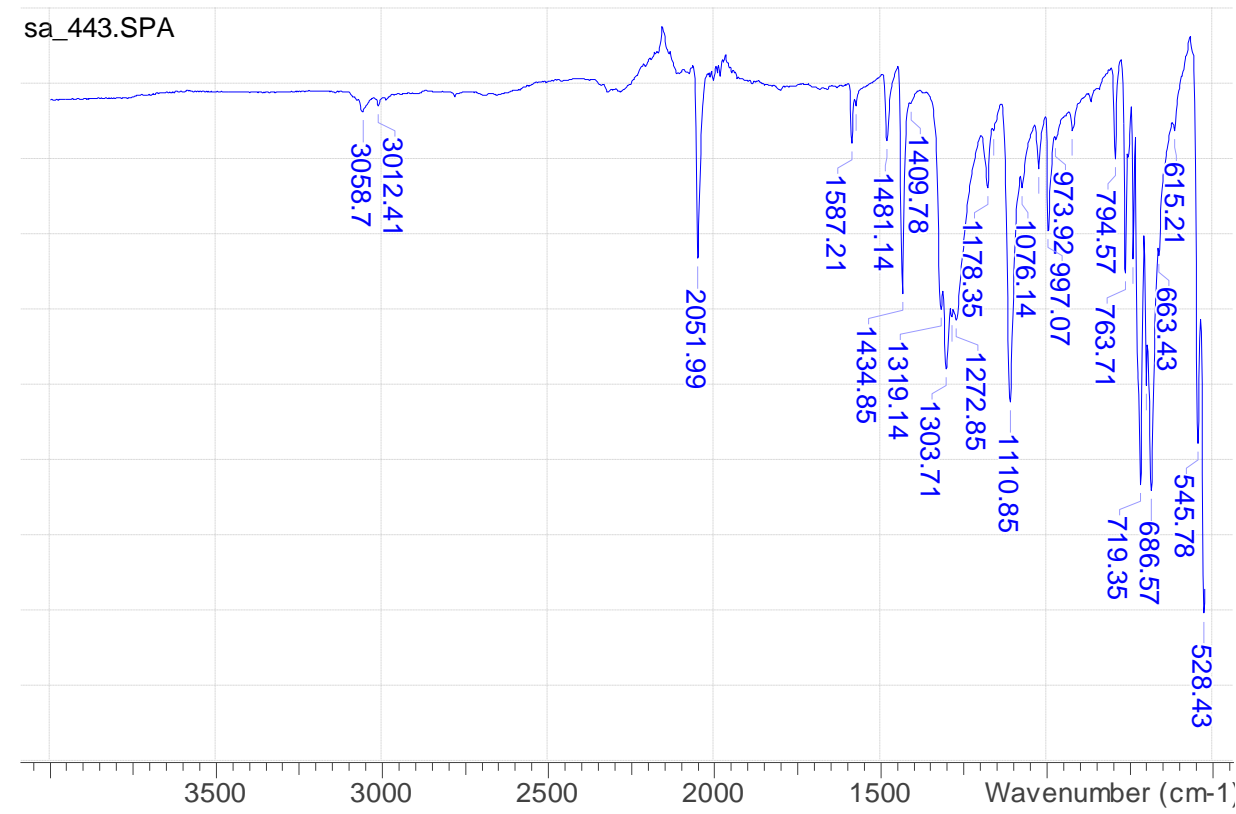




\section{RAMAN}

sa_443_1_(633_D00_x10_20s_20acc).spc

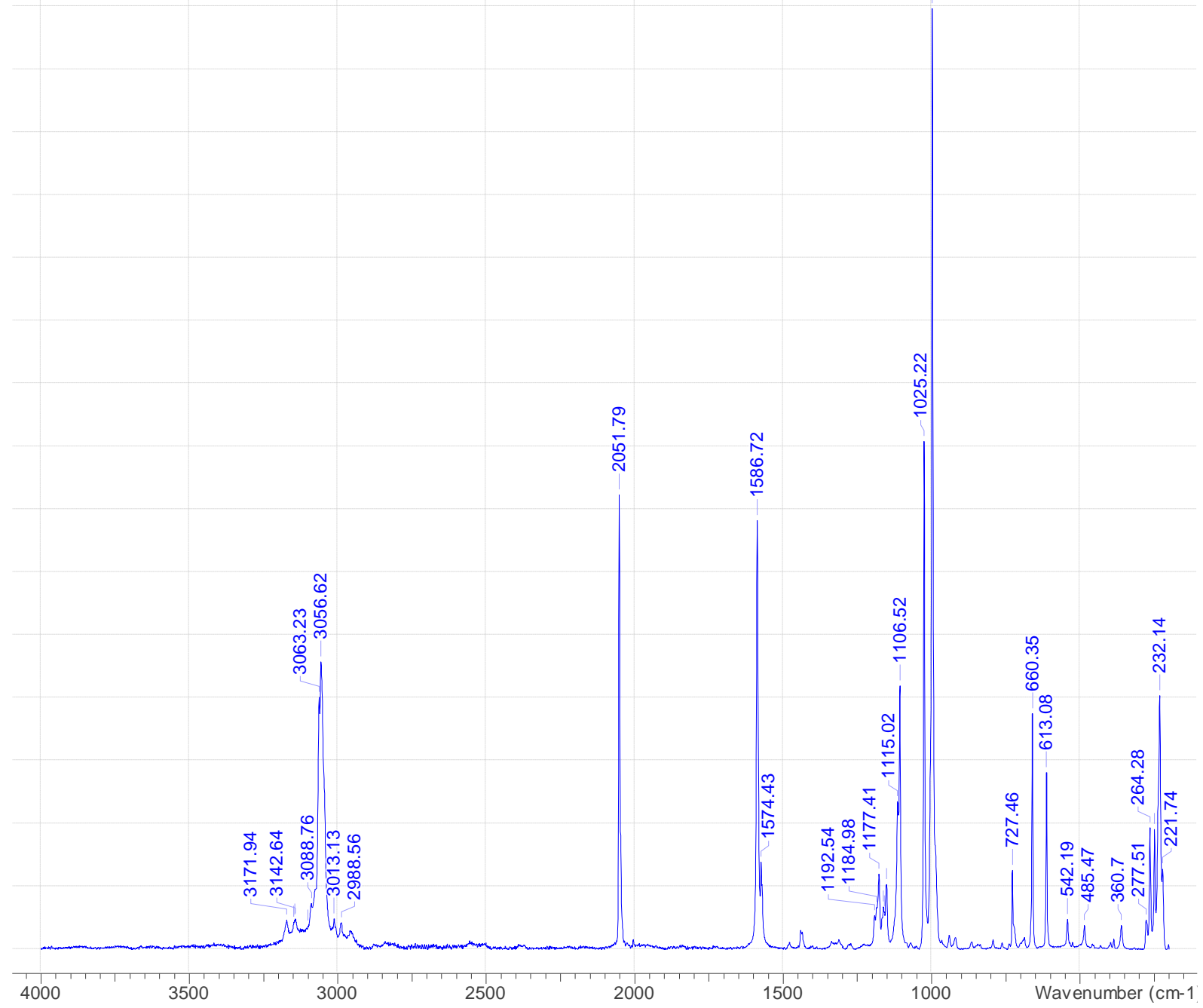




\section{${ }^{1} \mathrm{H}$ NMR}

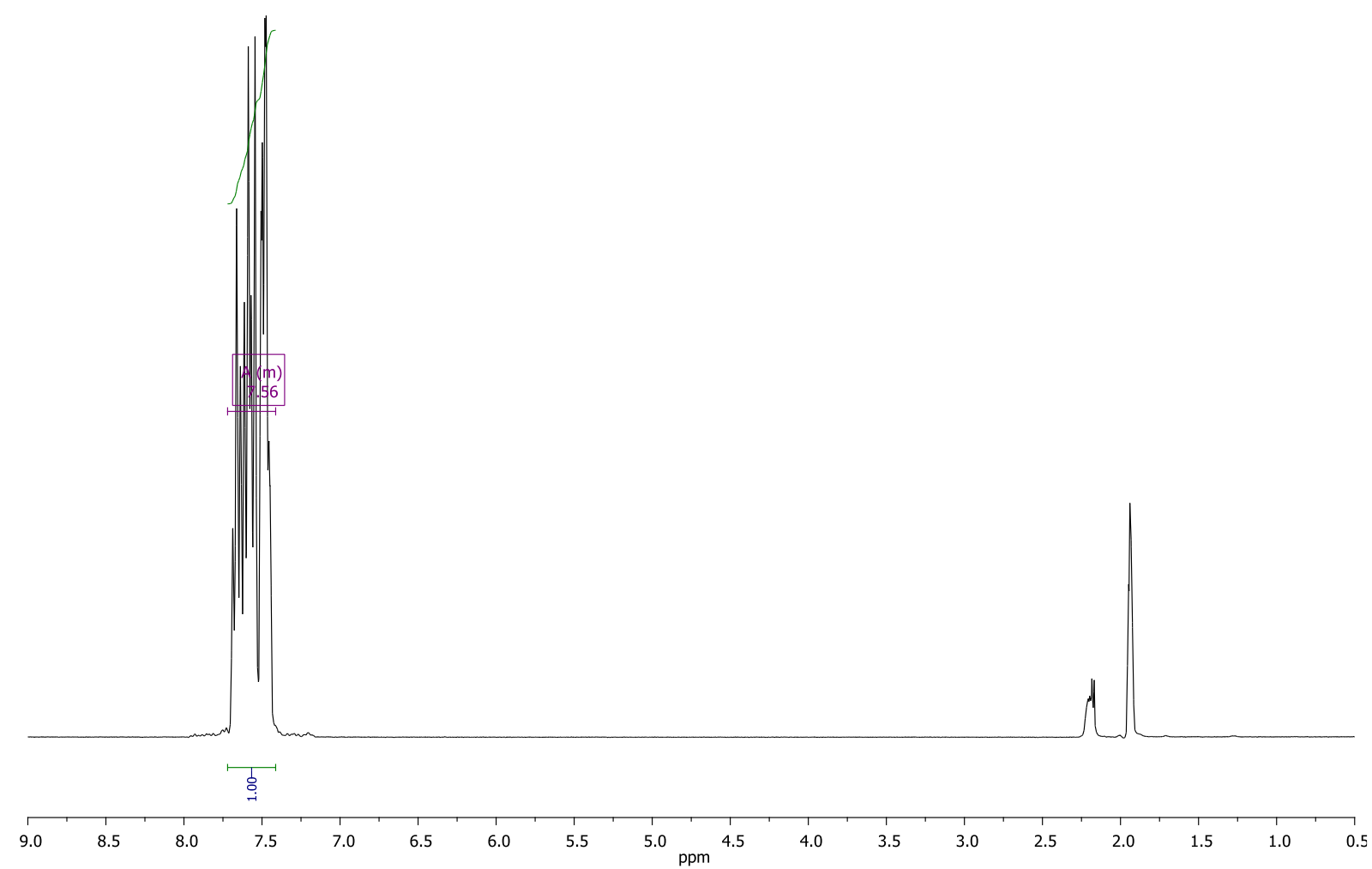

${ }^{13} \mathrm{C}$ NMR

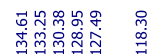

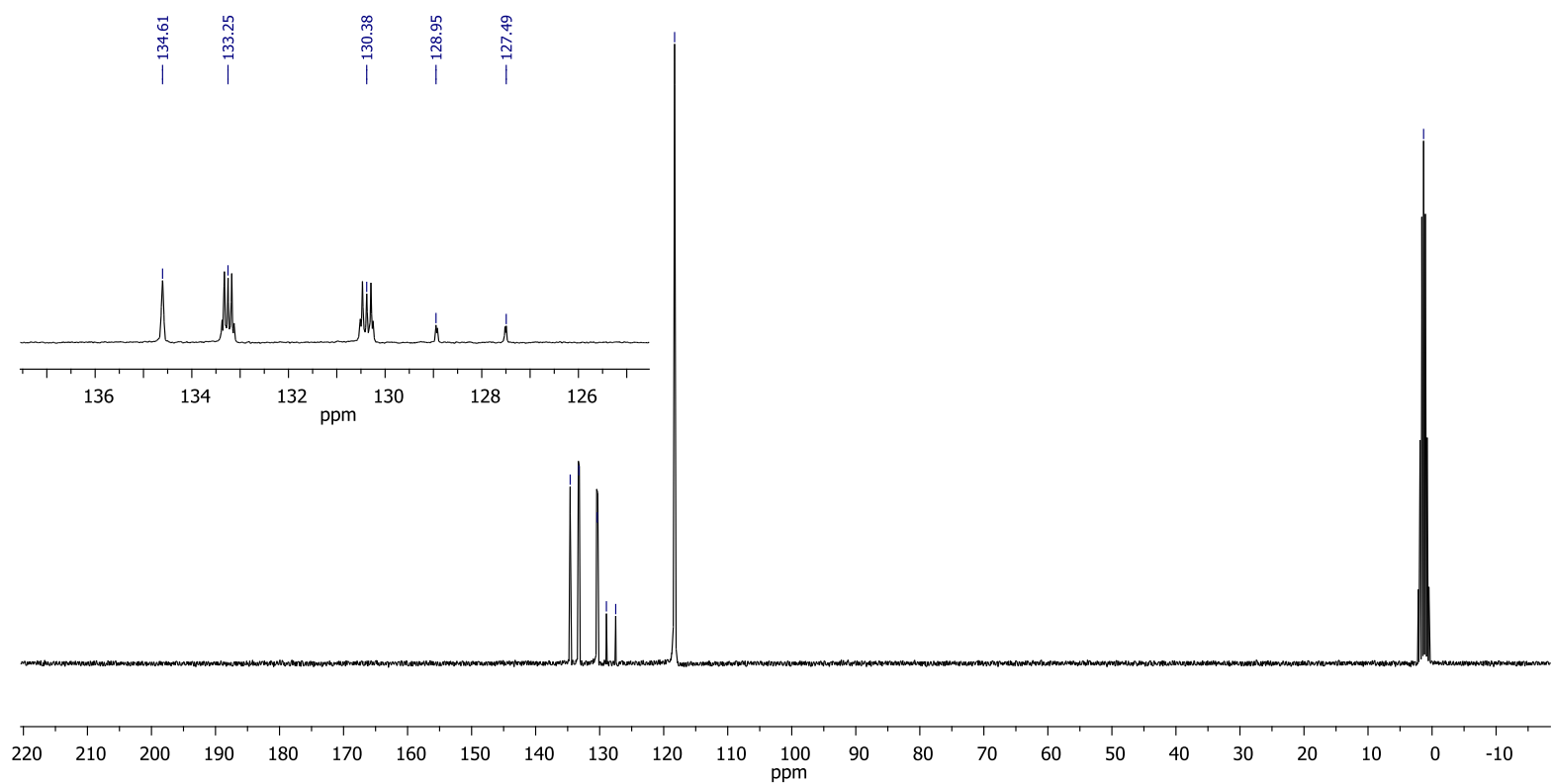


${ }^{31} \mathrm{P}$ NMR

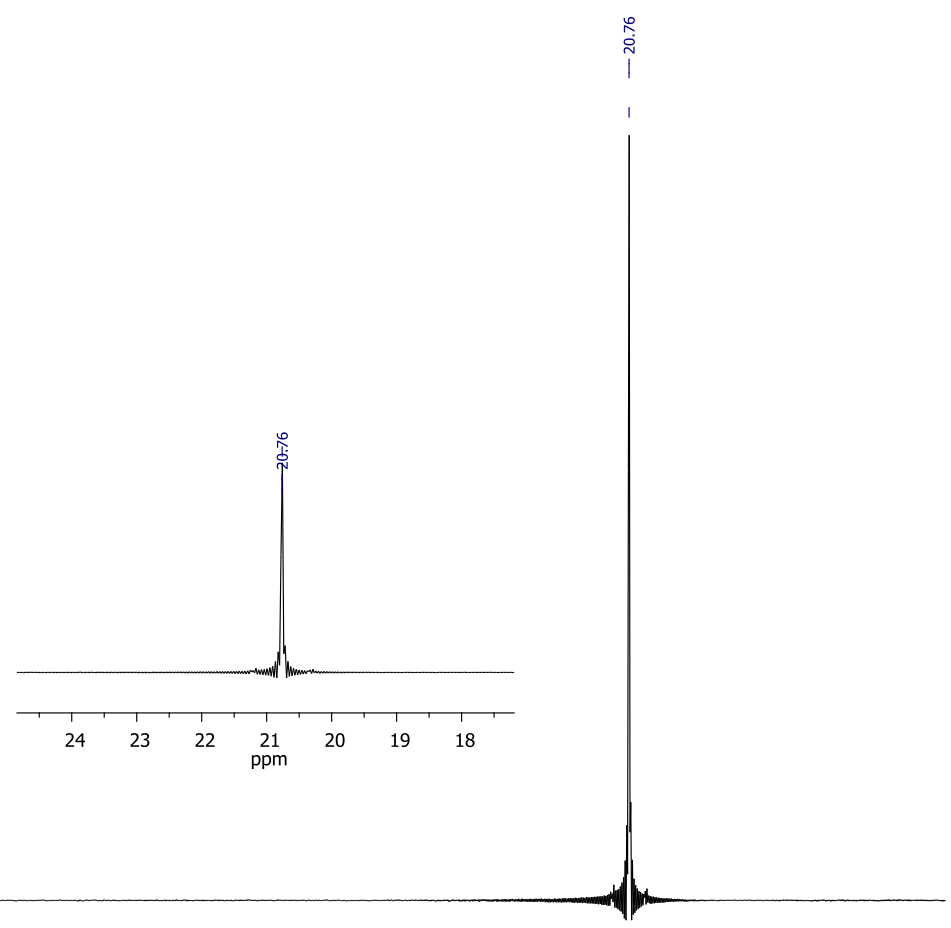

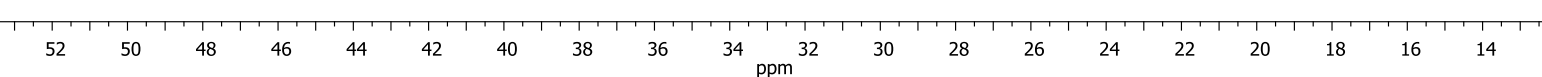


$[\mathrm{PPN}]\left[\mathrm{H}(\mathrm{NCS})_{2}\right](2)$

\section{TGA/DSC}

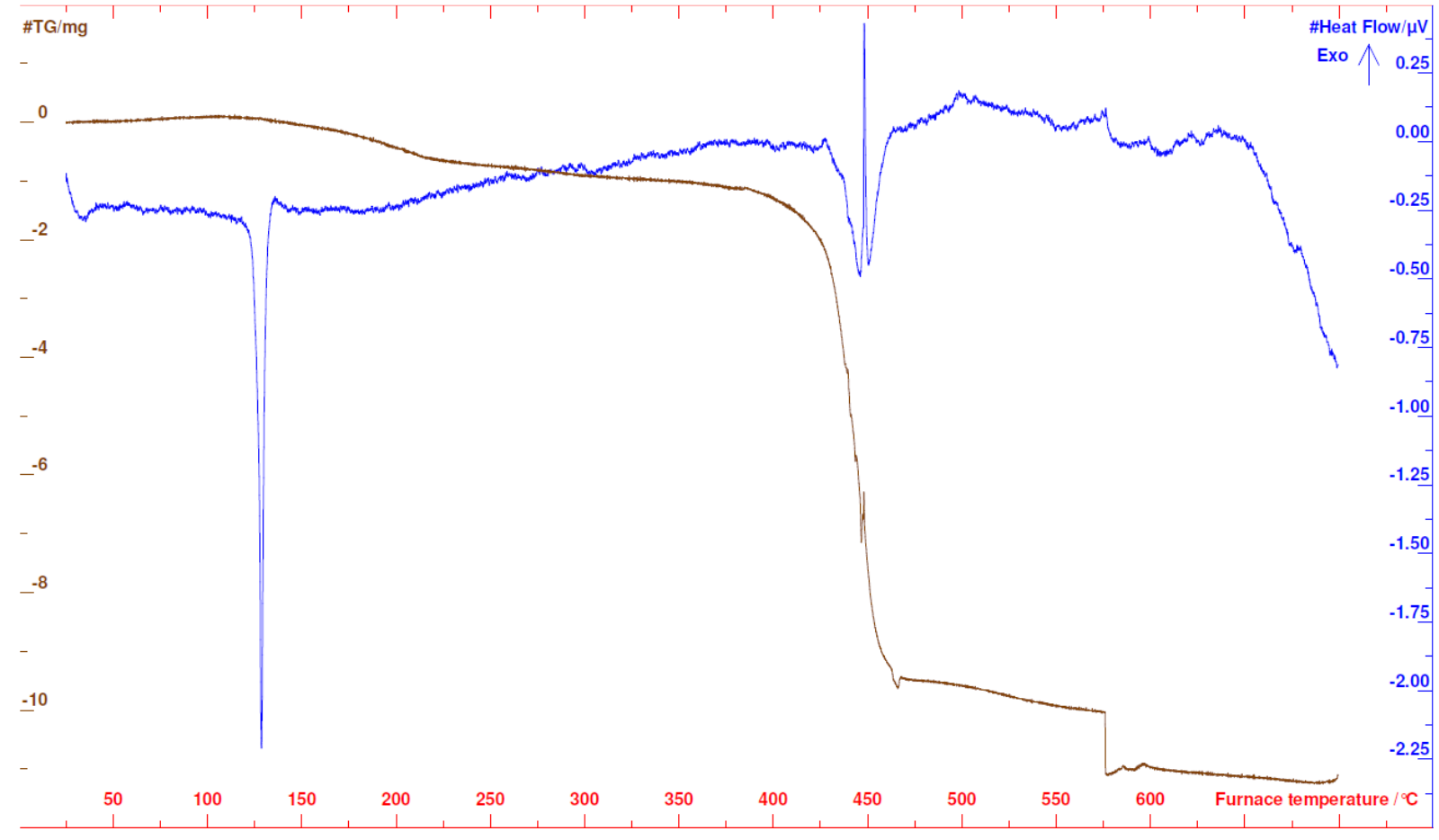

IR

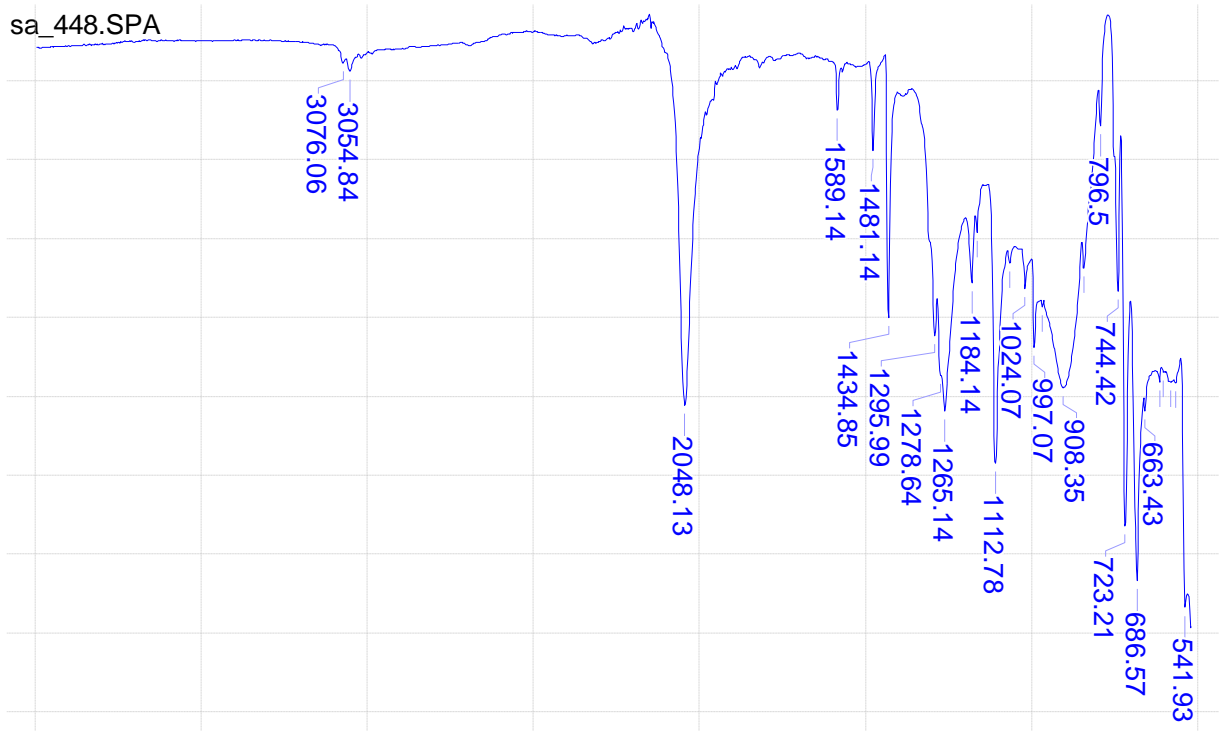

3500

3000

2500

2000

1500

Wavenumber $(\mathrm{cm}-1$ 


\section{RAMAN}

sa_448_1_(633_D00_x10_20s_20acc).spc

今.

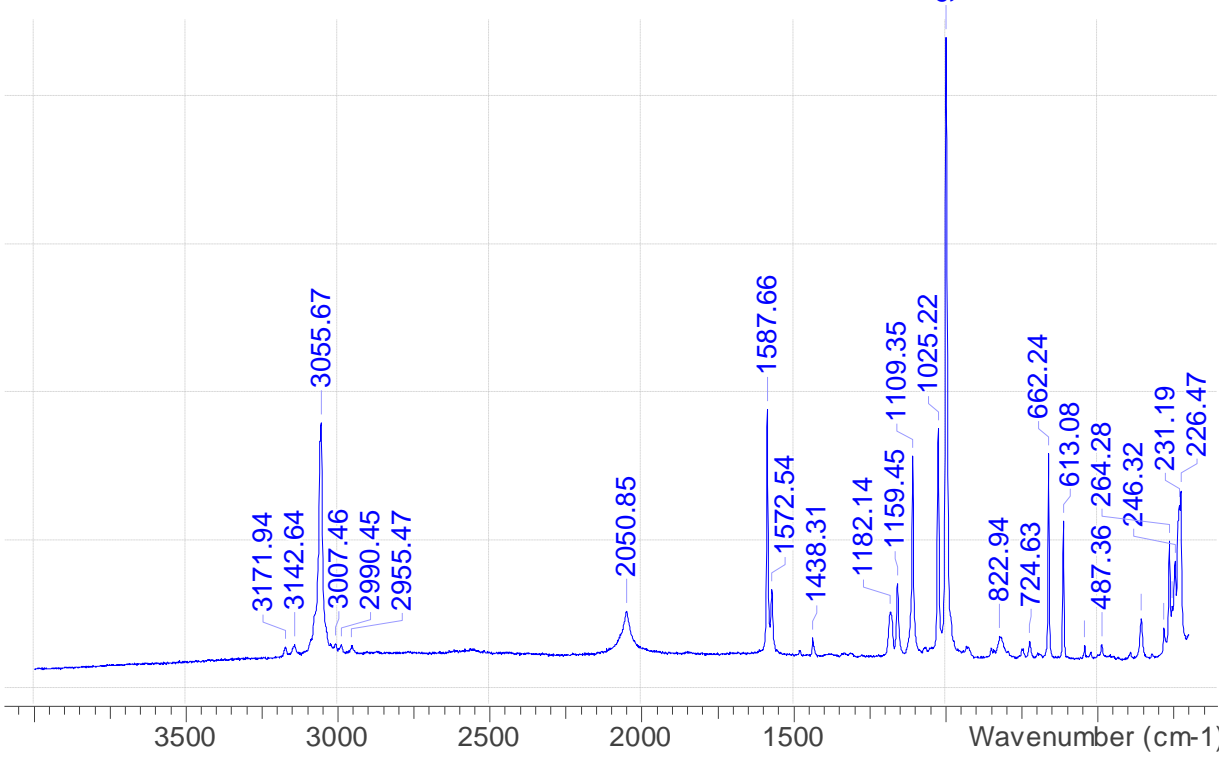

${ }^{1} \mathrm{H}$ NMR

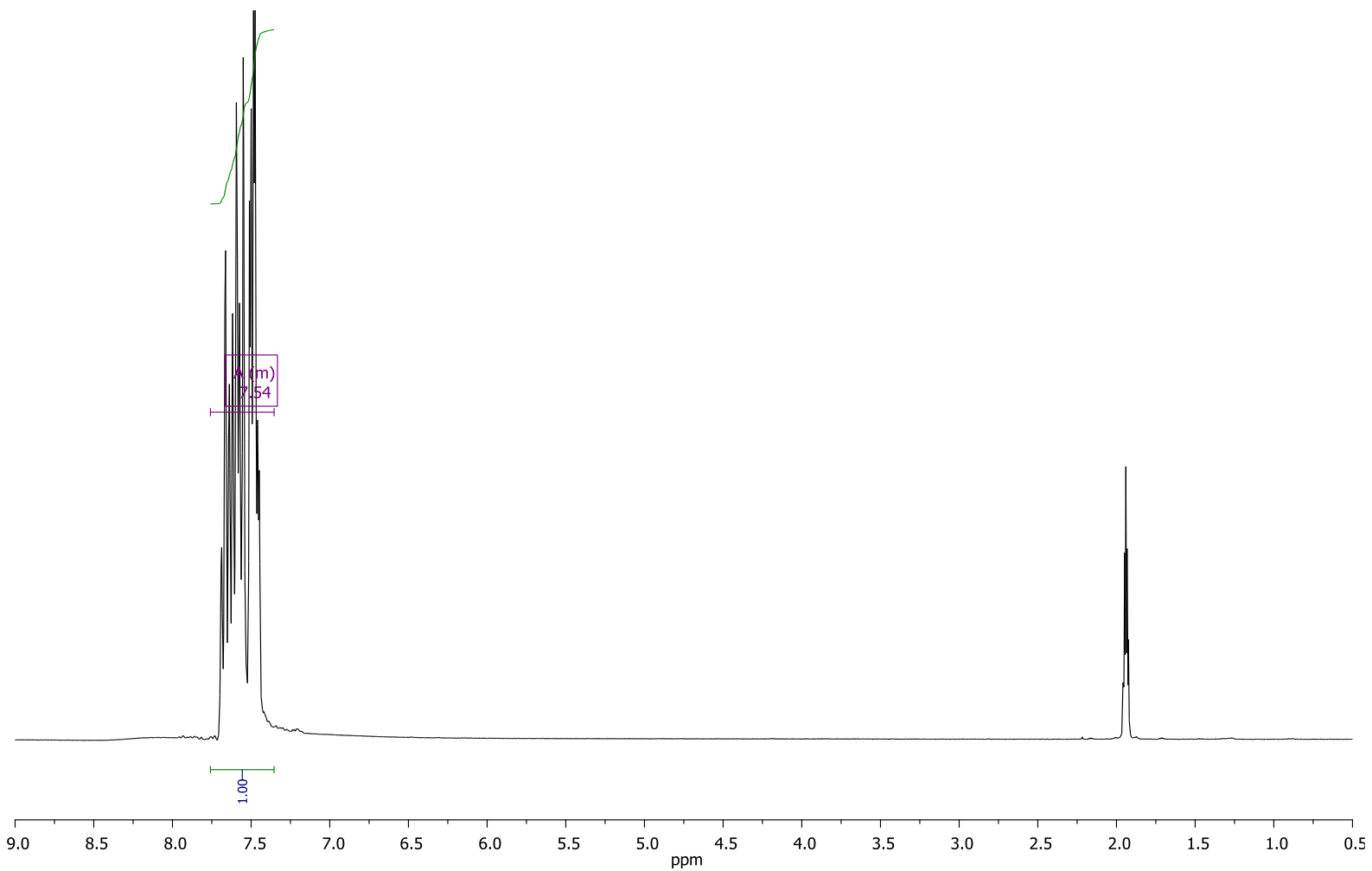




\section{${ }^{13} \mathrm{C}$ NMR}

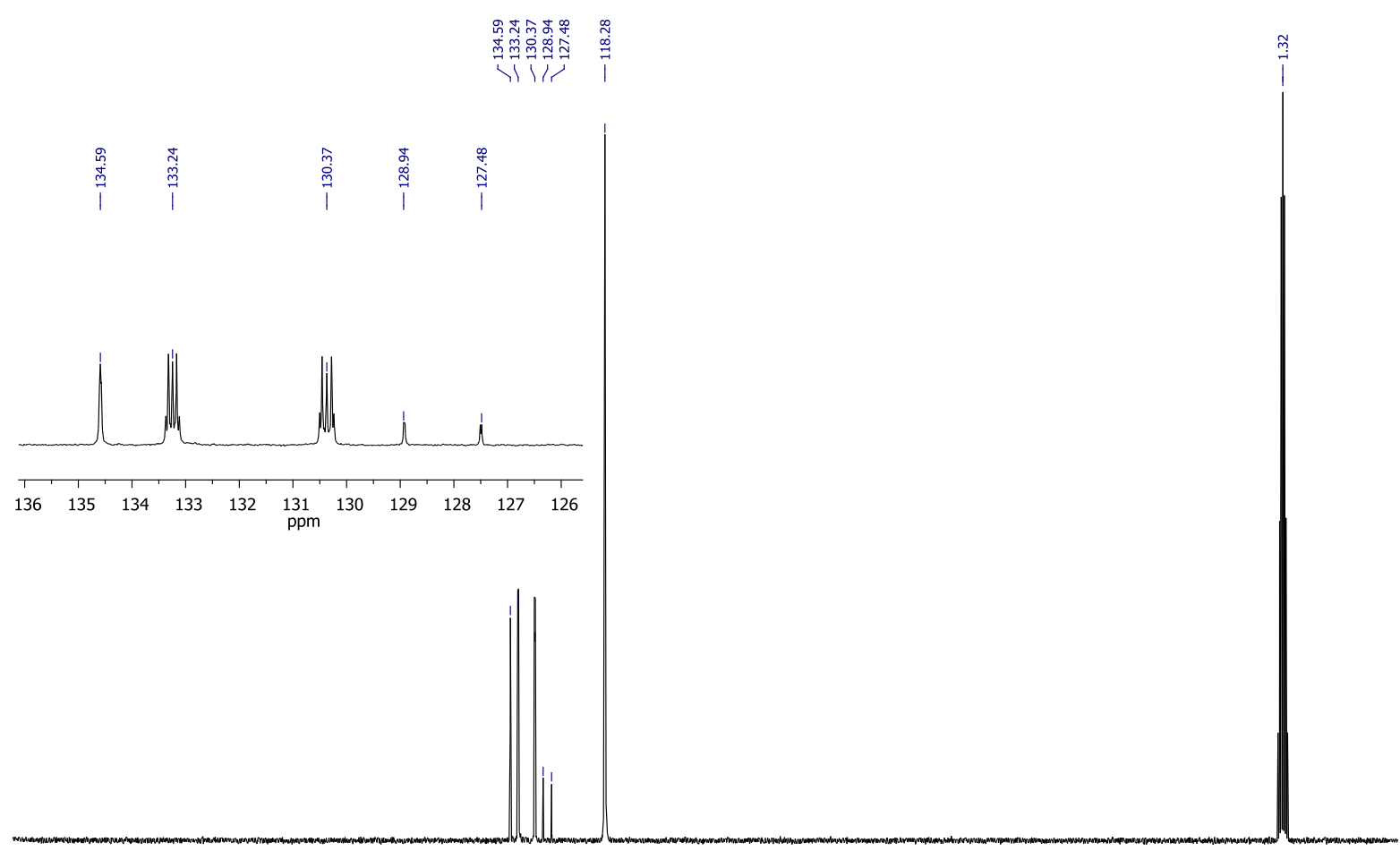

\begin{tabular}{rlllllllllllllllllllllllll}
\hline & 220 & 210 & 200 & 190 & 180 & 170 & 160 & 150 & 140 & 130 & 120 & 110 & $\begin{array}{l}100 \\
\mathrm{ppm}\end{array}$ & 90 & 80 & 70 & 60 & 50 & 40 & 30 & 20 & 10 & 0 & -10
\end{tabular}

${ }^{31} \mathrm{P}$ NMR
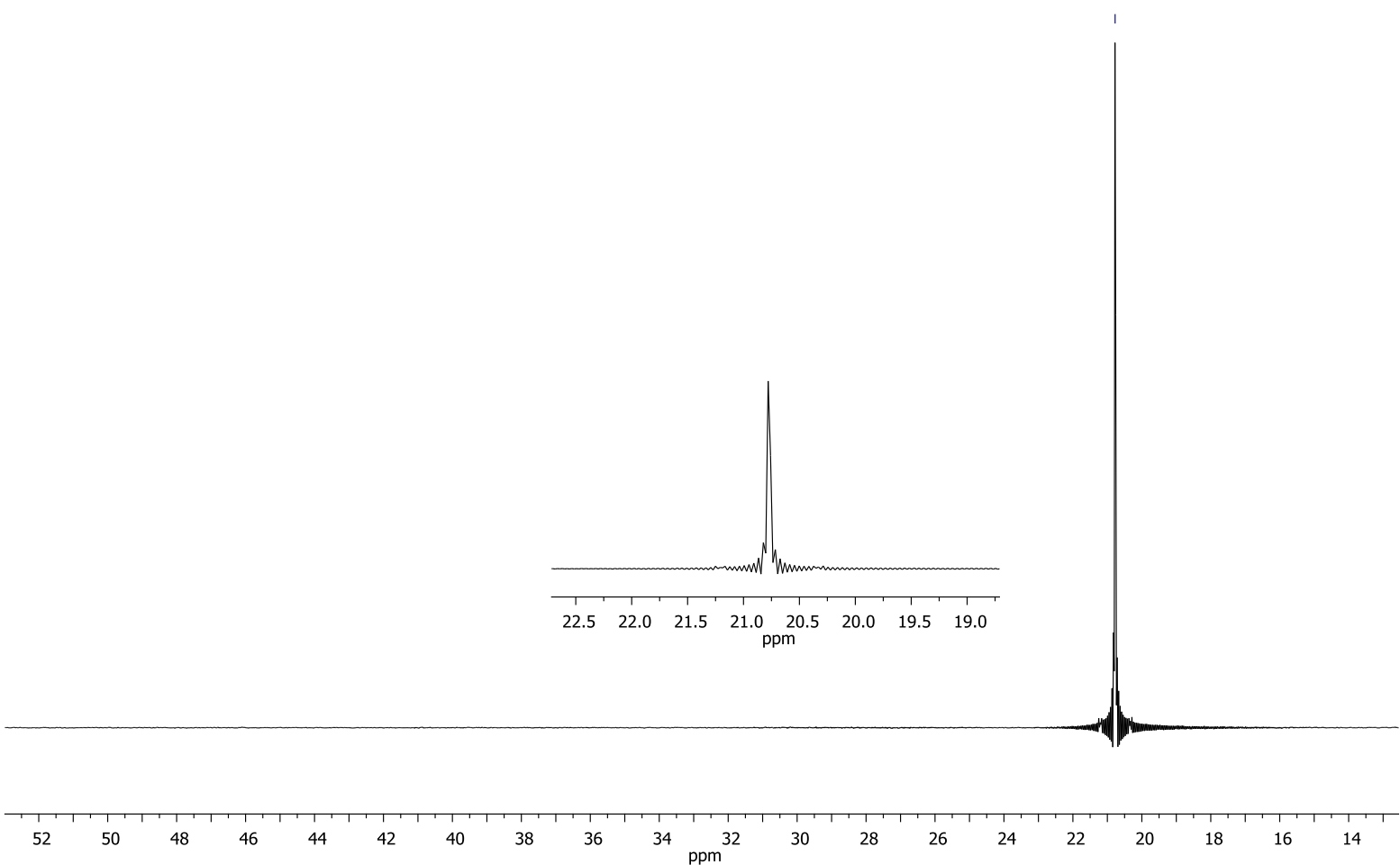
$\mathrm{Sb}(\mathrm{NCS})_{3} / \mathrm{Sb}(\mathrm{NCS})_{3} \cdot \operatorname{thf}(\mathbf{3})$

\section{TGA/DSC}

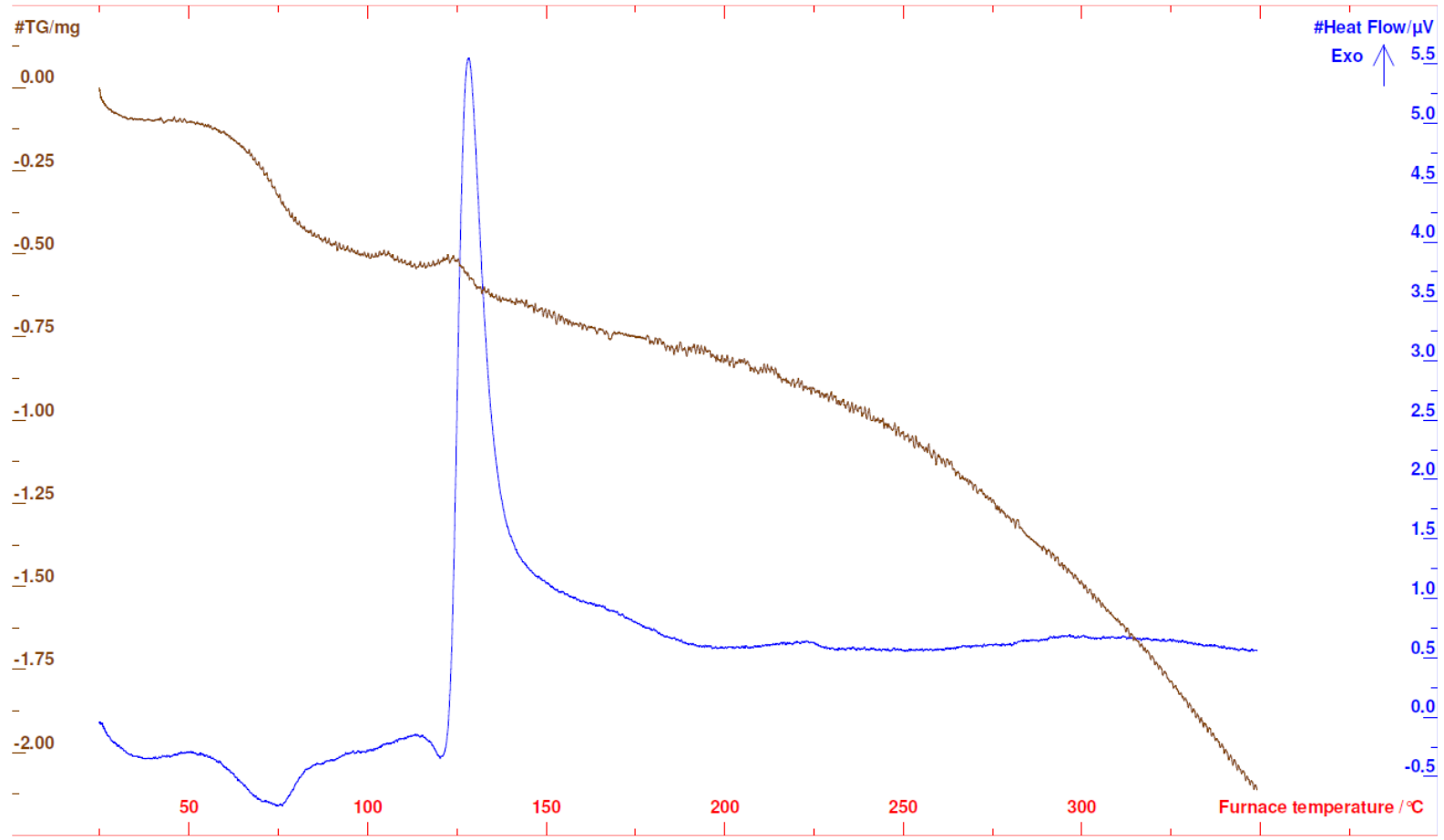

IR

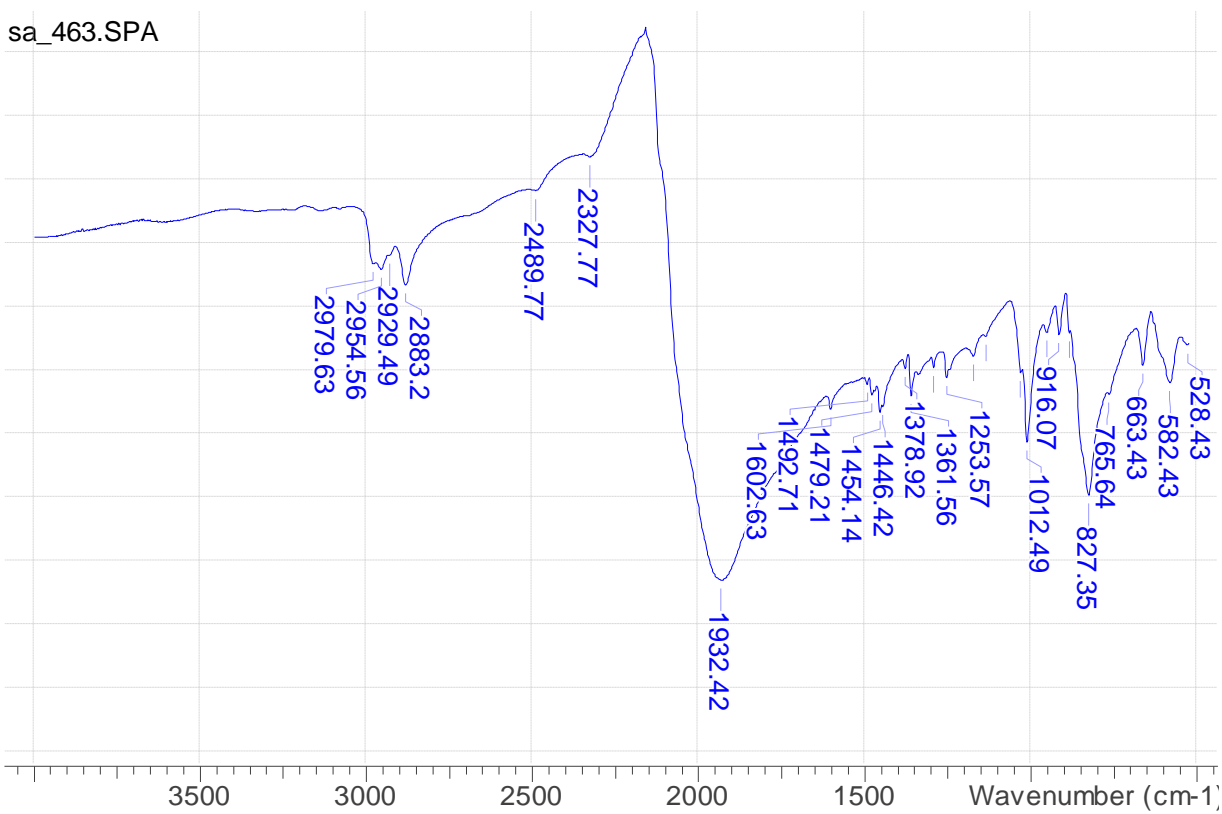




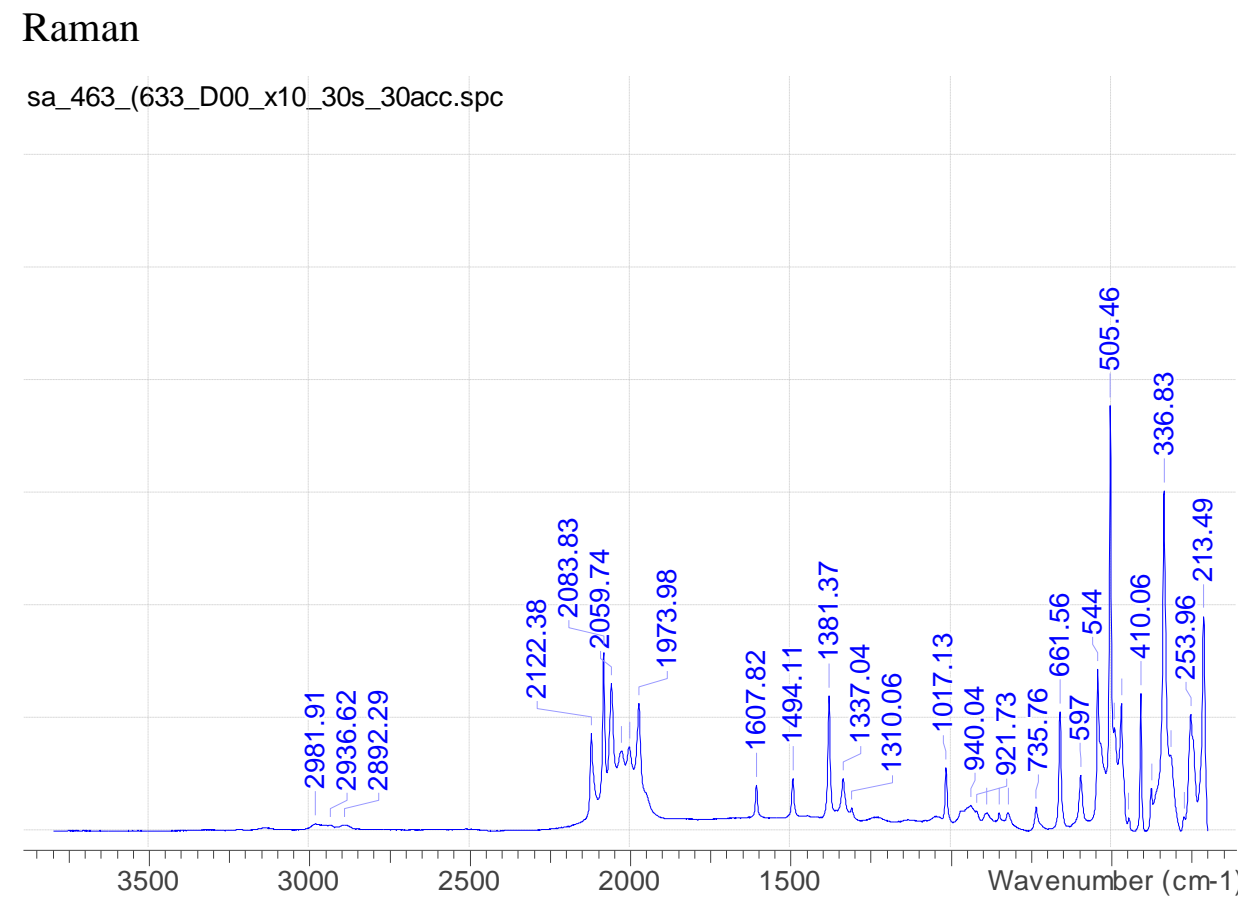


$\mathrm{Bi}(\mathrm{NCS})_{3} \cdot \operatorname{thf}(\mathbf{4})$

TGA/DSC

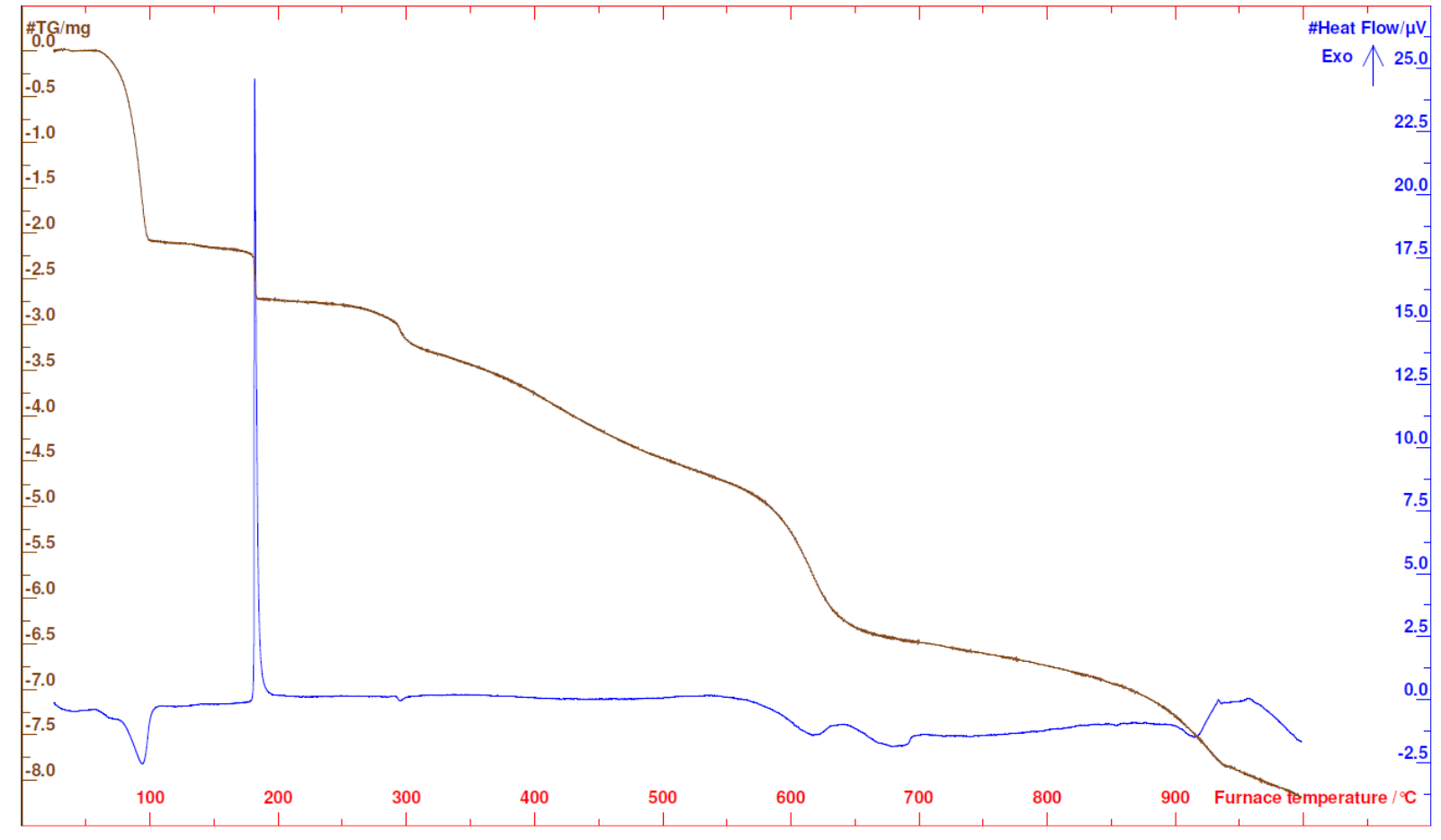


IR

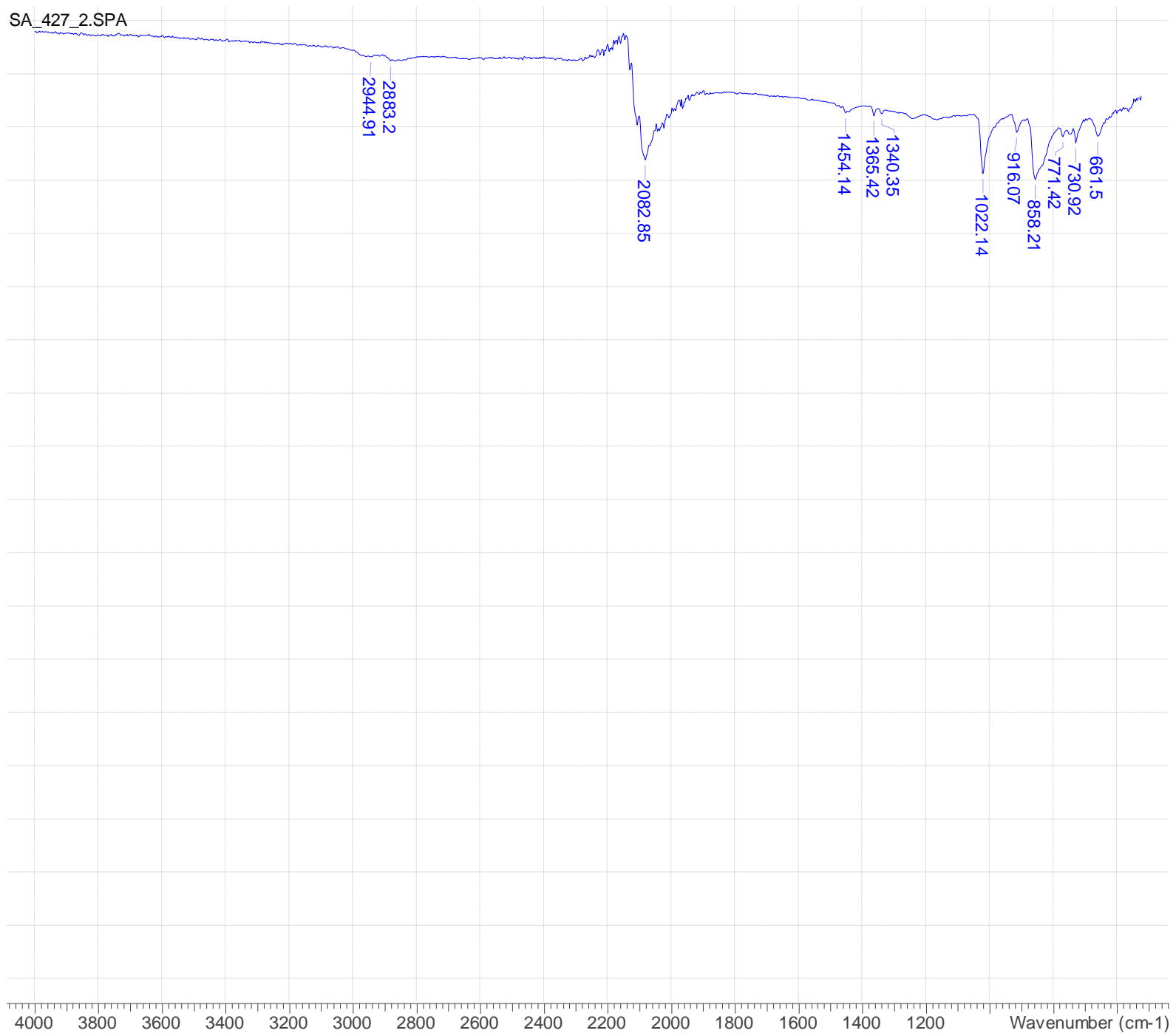

RAMAN

sa_427_2_(633_D06_x10_10sec_20acc).spc

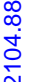

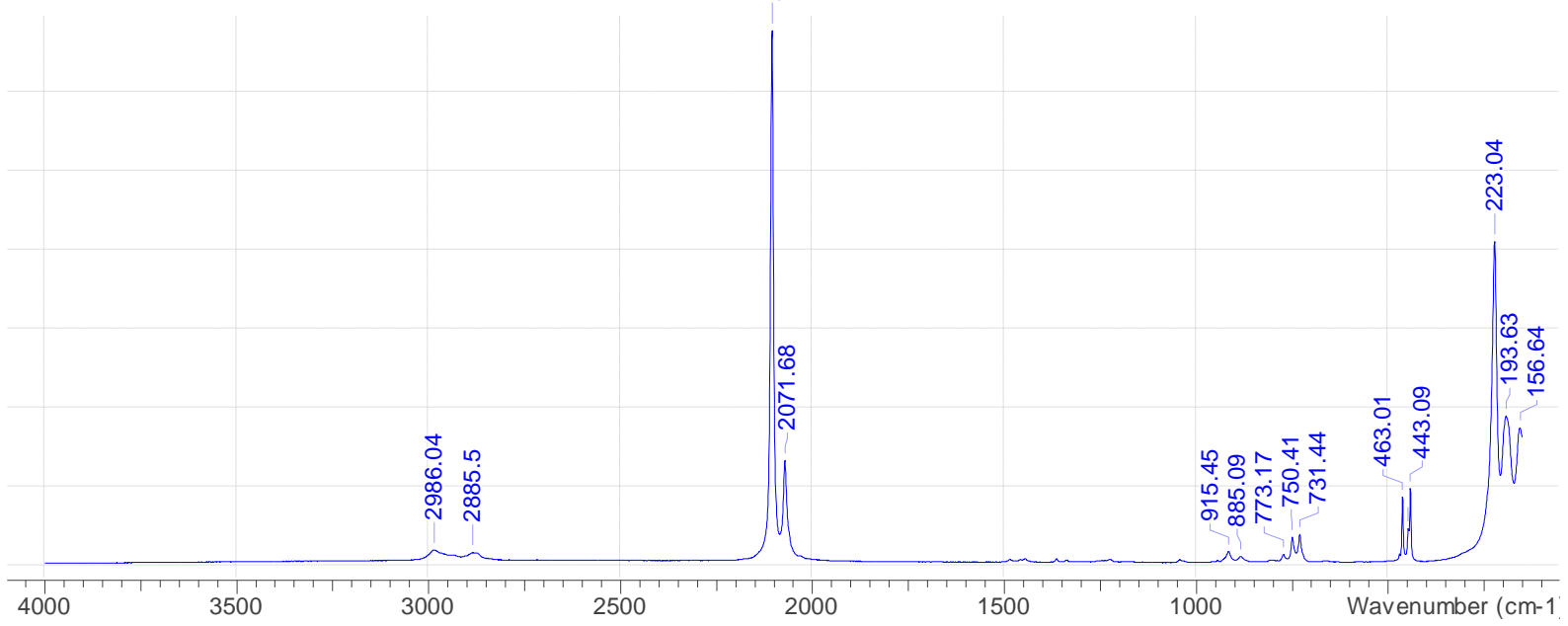


${ }^{1} \mathrm{H}$ NMR

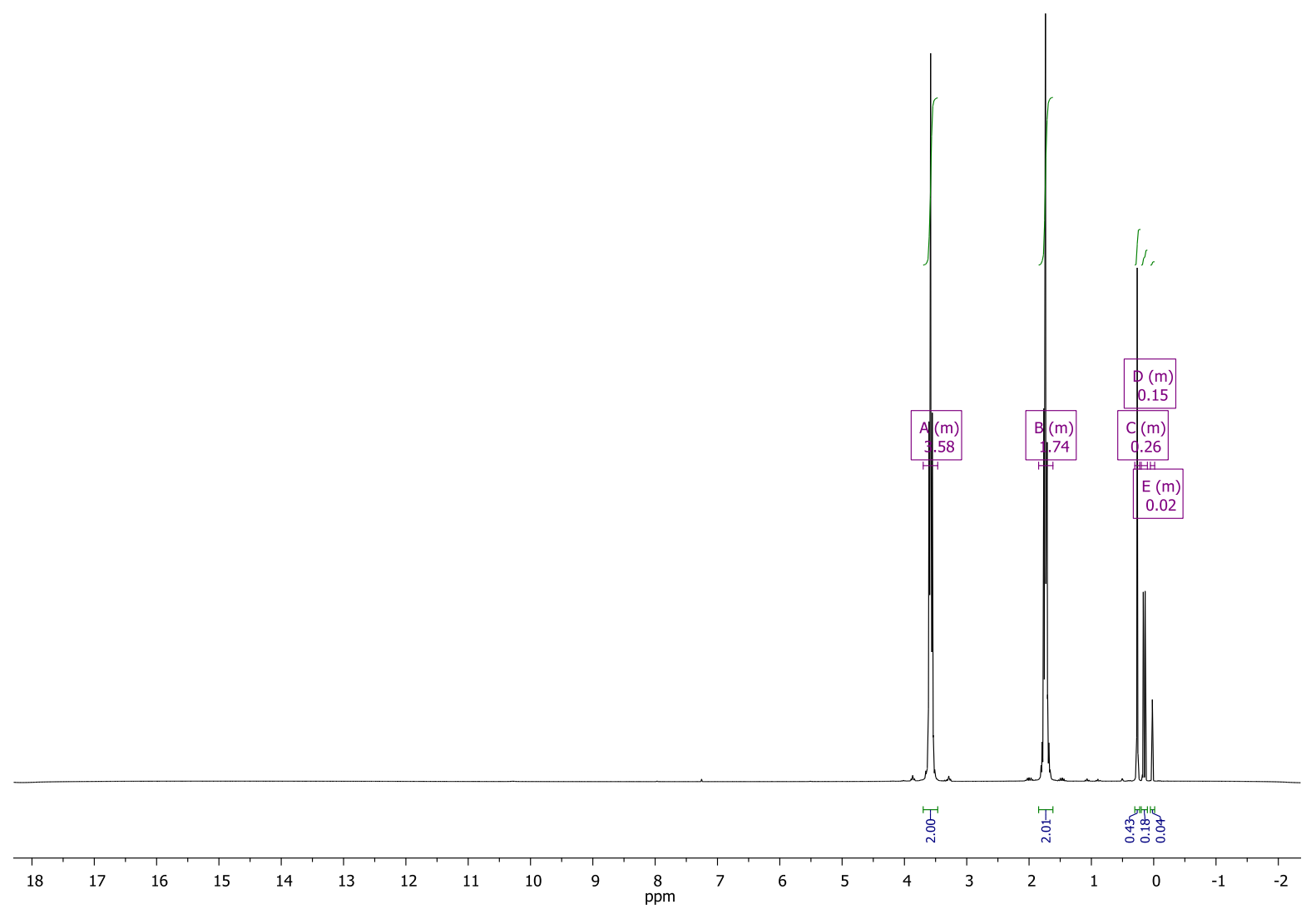

${ }^{13} \mathrm{C}$ NMR

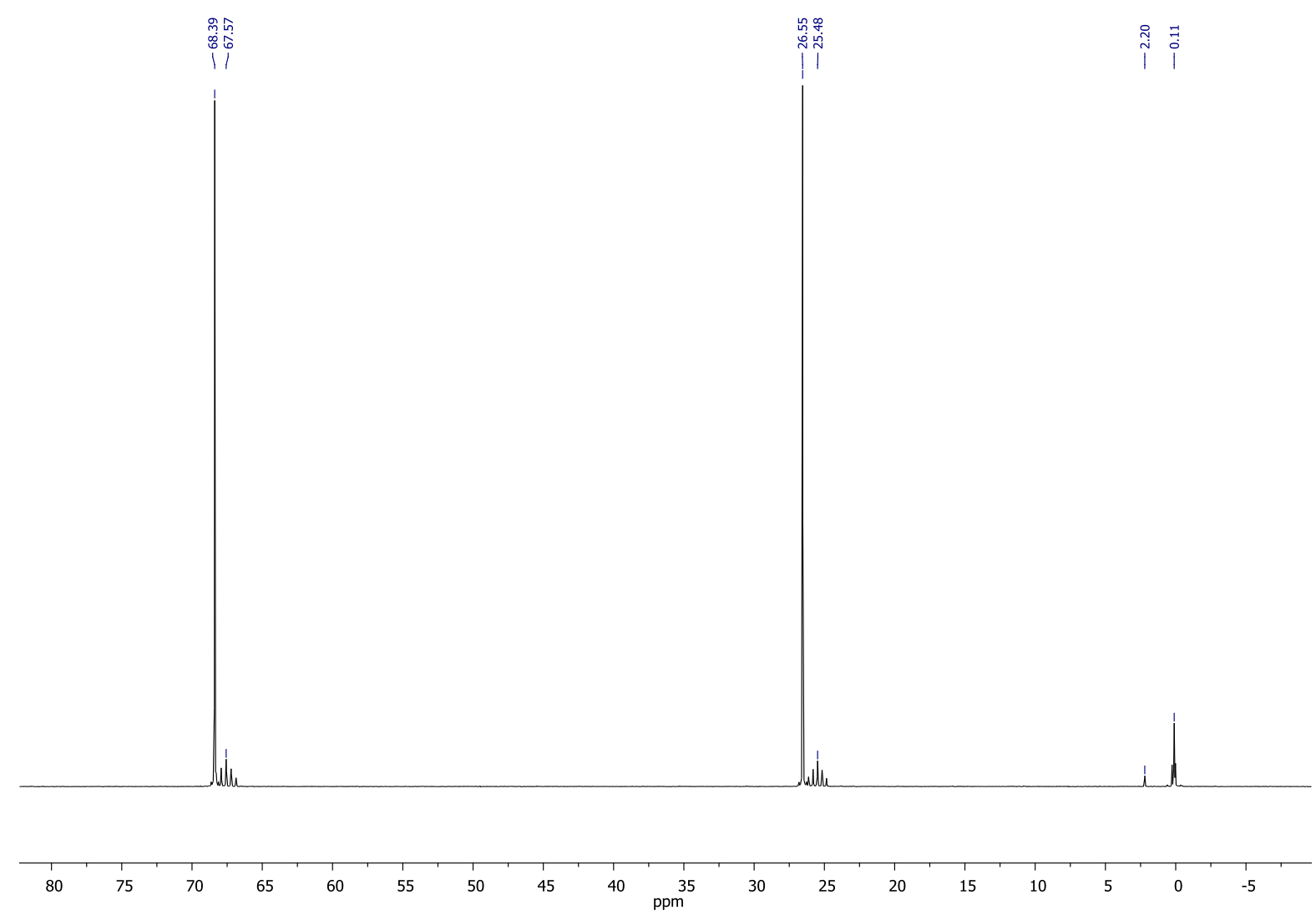


$[\mathrm{PPN}]_{2}\left[\mathrm{Sb}(\mathrm{NCS})_{5}\right](\mathbf{5})$

TGA/DSC

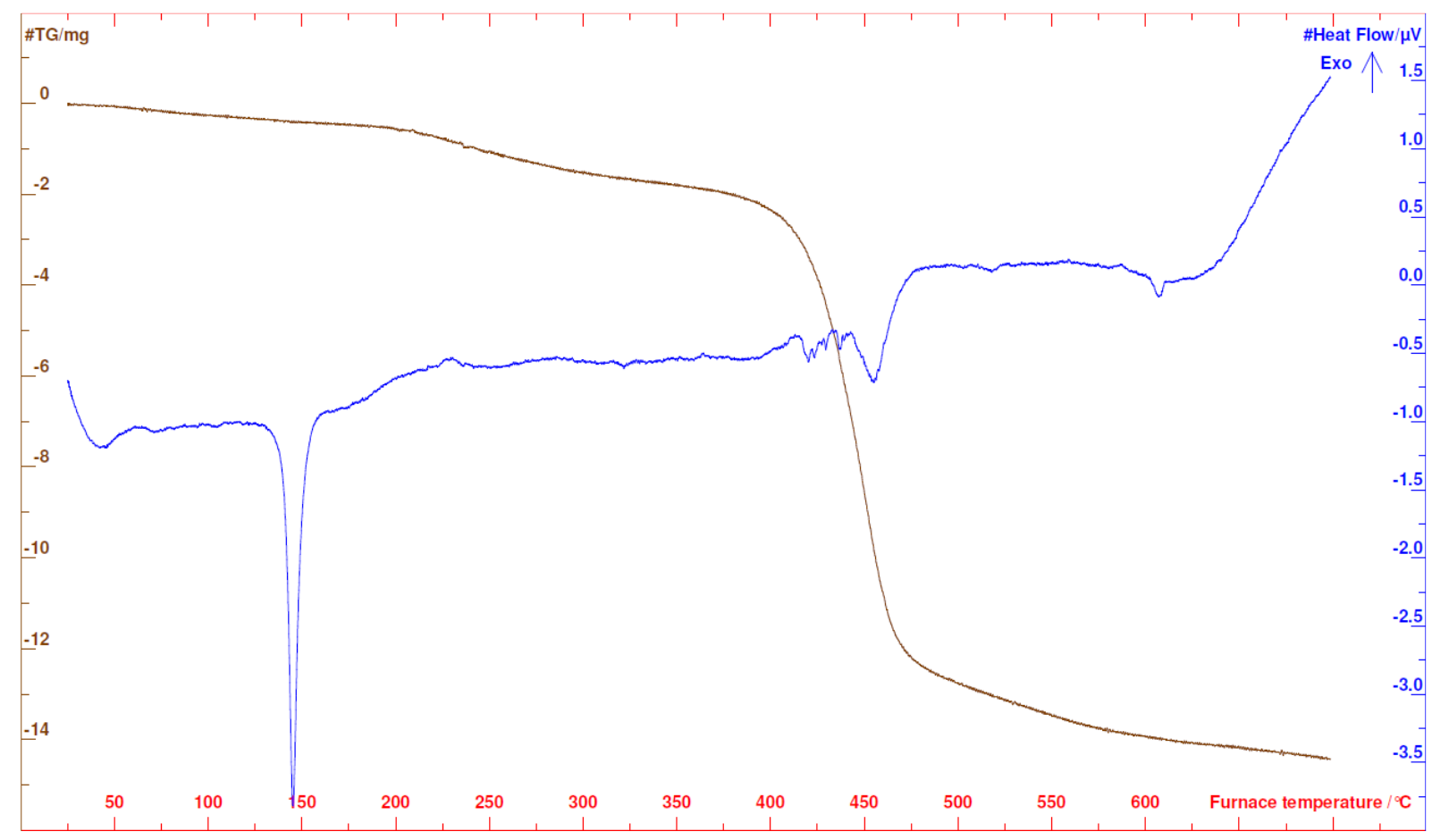


IR

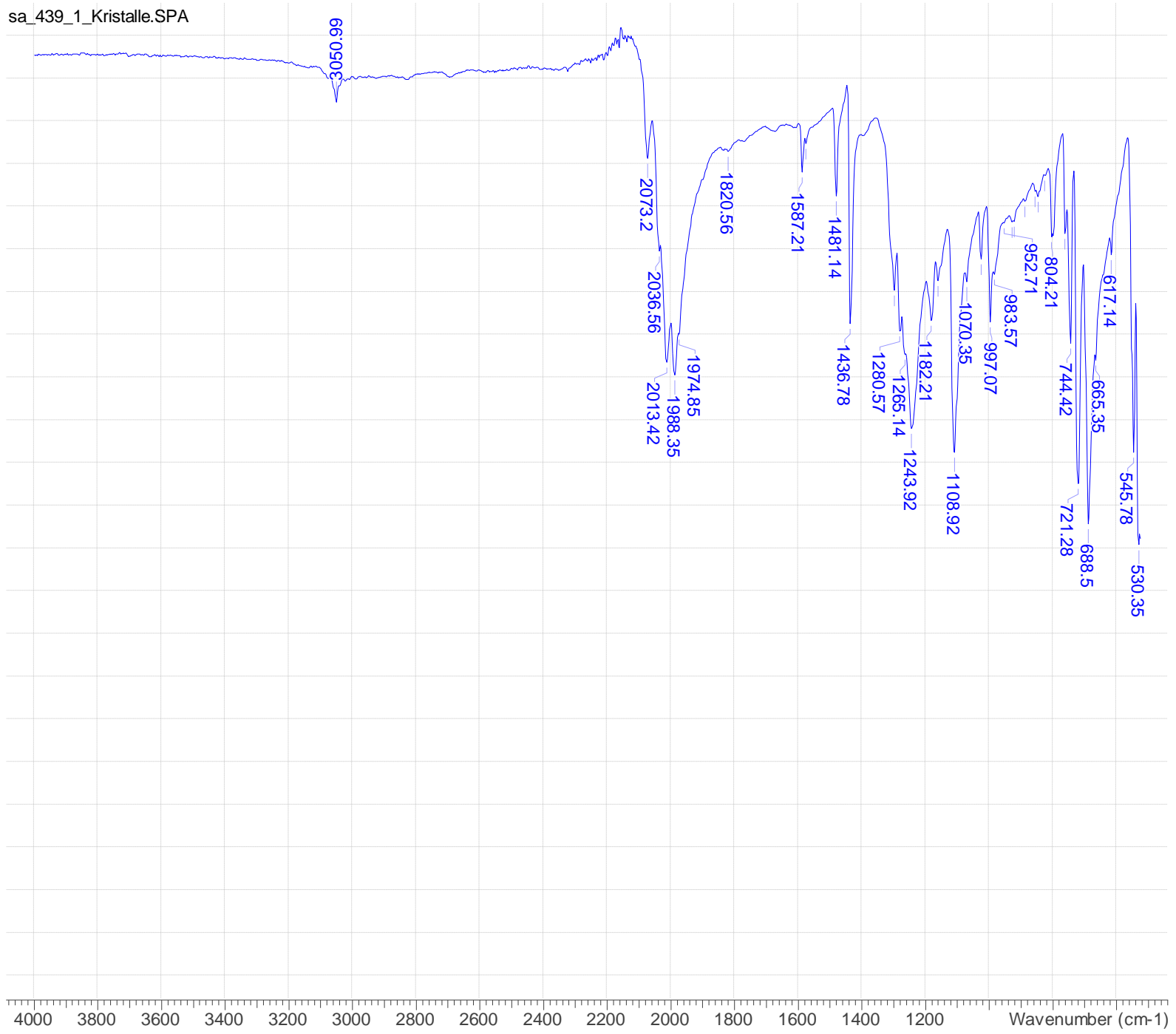




\section{RAMAN}

sa_439_1(abgeschmolzen)_(633_D03_x10_10s_20acc)

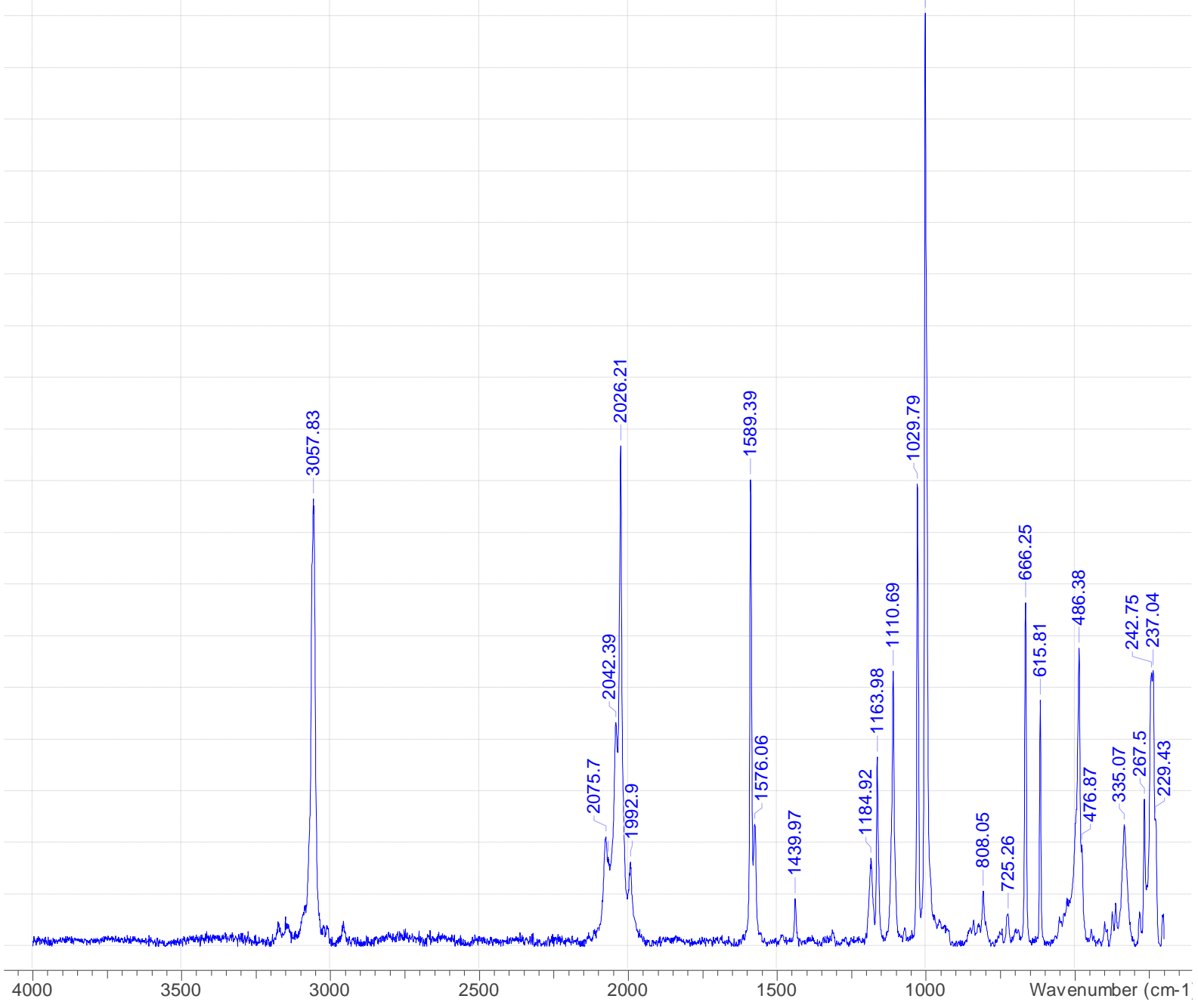


${ }^{1} \mathrm{H}$ NMR

$\stackrel{8}{i}$

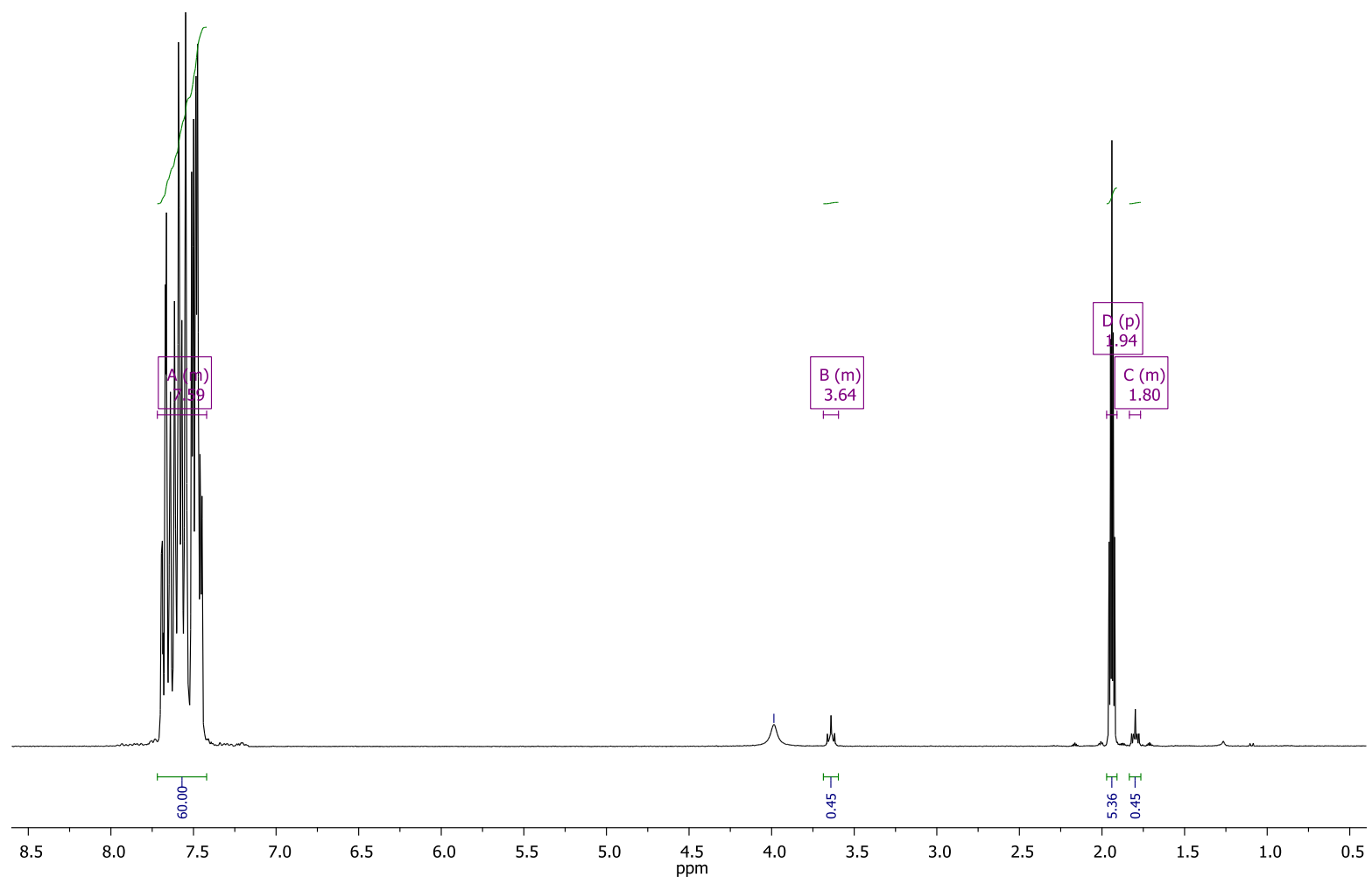

${ }^{13} \mathrm{C}$ NMR

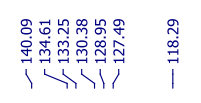
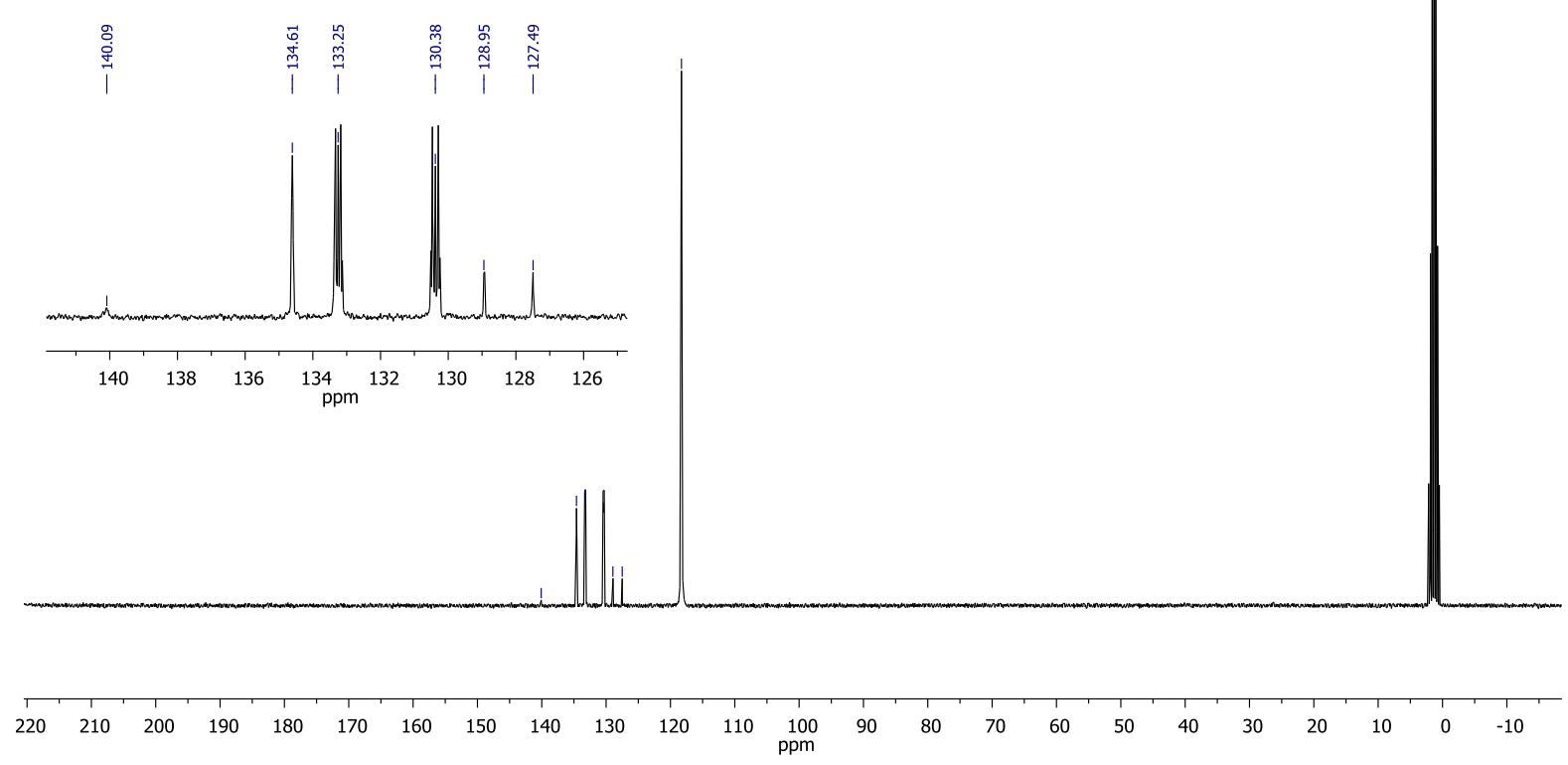

S46 


\section{${ }^{13} \mathrm{C}$ IG NMR}

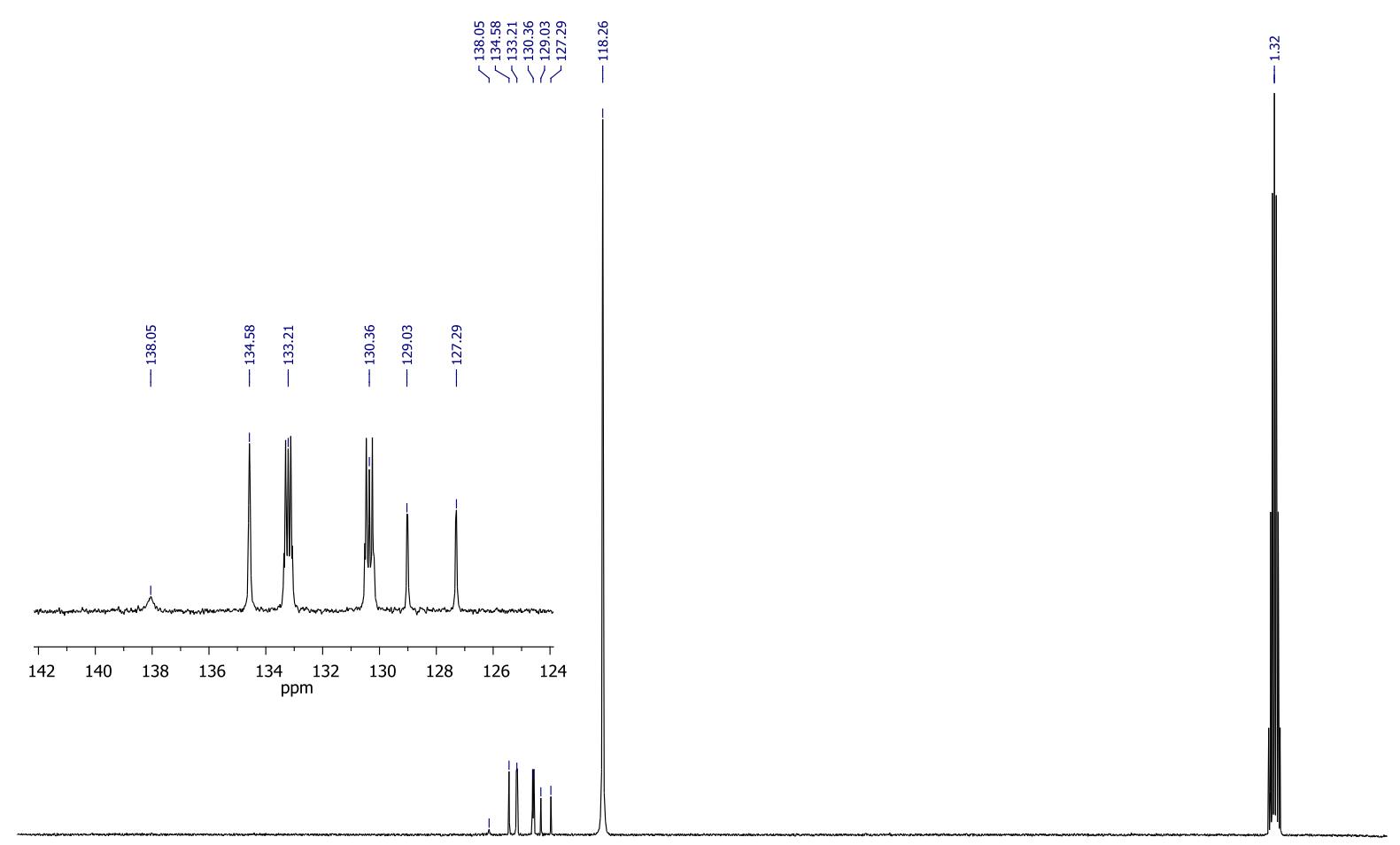

\begin{tabular}{rlllllllllllllllllllllllllllll}
\hline & 220 & 210 & 200 & 190 & 180 & 170 & 160 & 150 & 140 & 130 & 120 & 110 & 100 & 90 & 80 & 70 & 60 & 50 & 40 & 30 & 20 & 10 & 0 & -10
\end{tabular}

${ }^{31} \mathrm{P}$ NMR

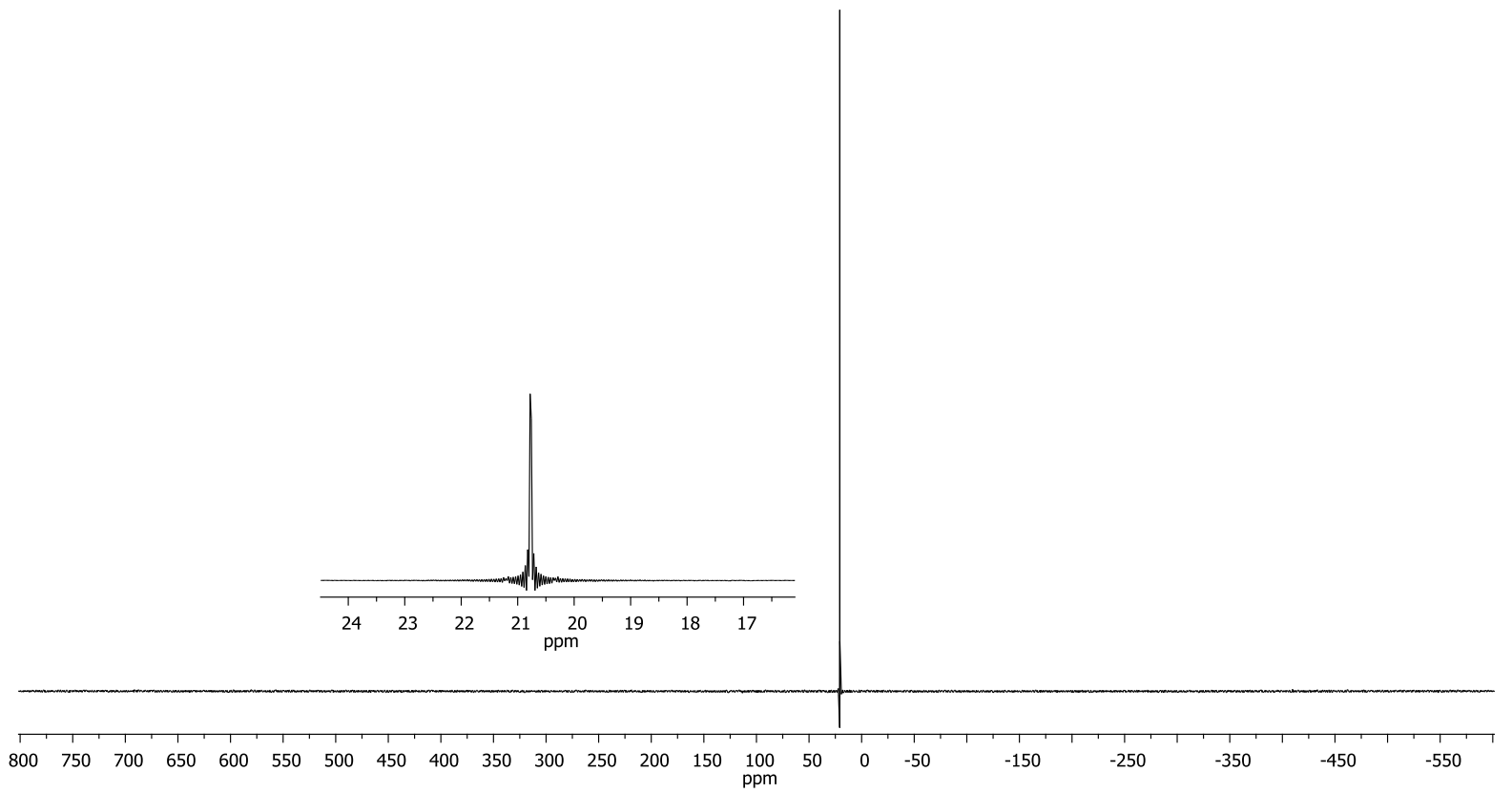


$[\mathrm{PPN}]_{2}\left[\mathrm{Bi}(\mathrm{NCS})_{3}(\mathrm{SCN})_{2}(\right.$ thf $\left.)\right](\mathbf{6})$

\section{TGA/DSC}

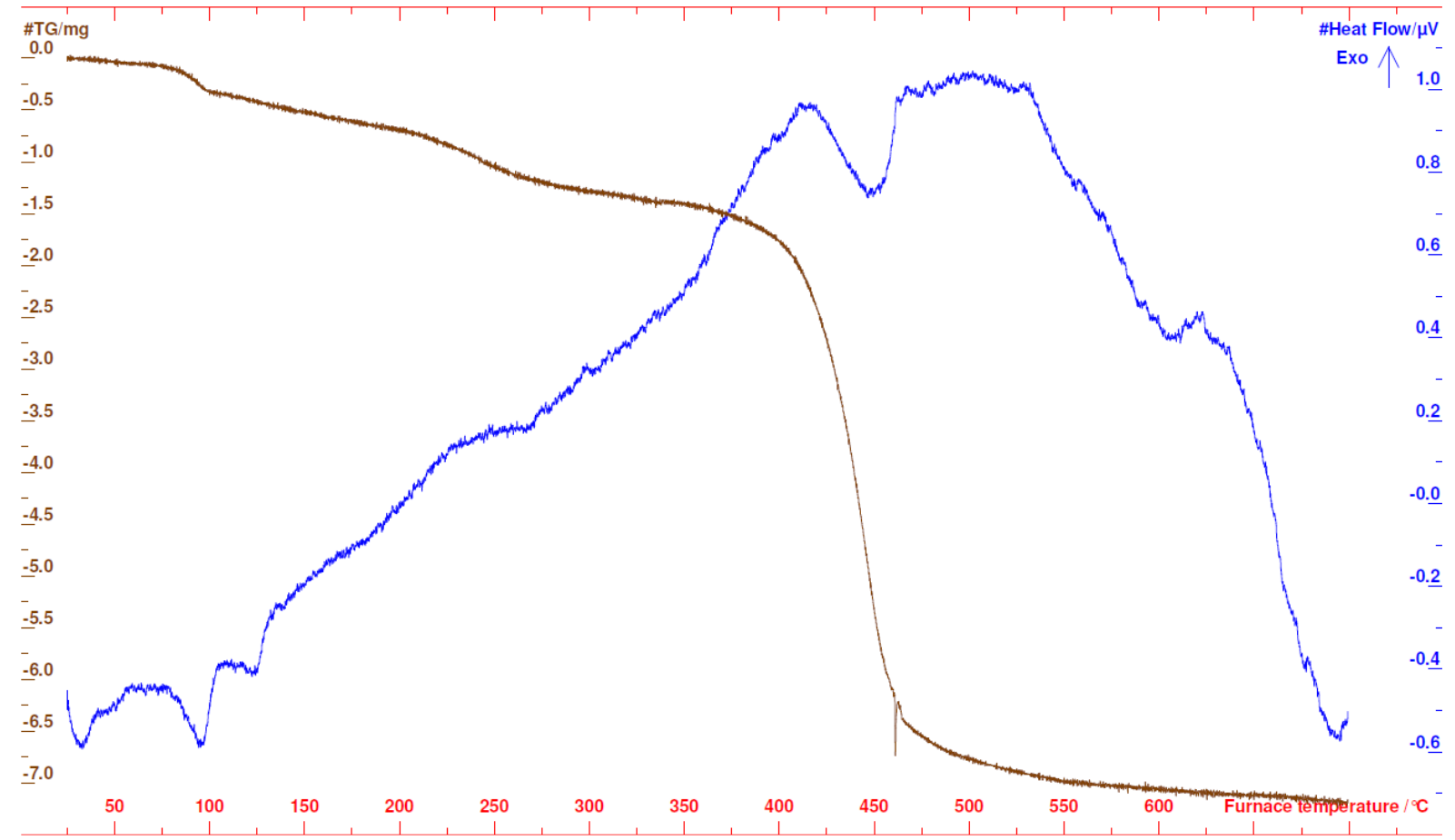

IR

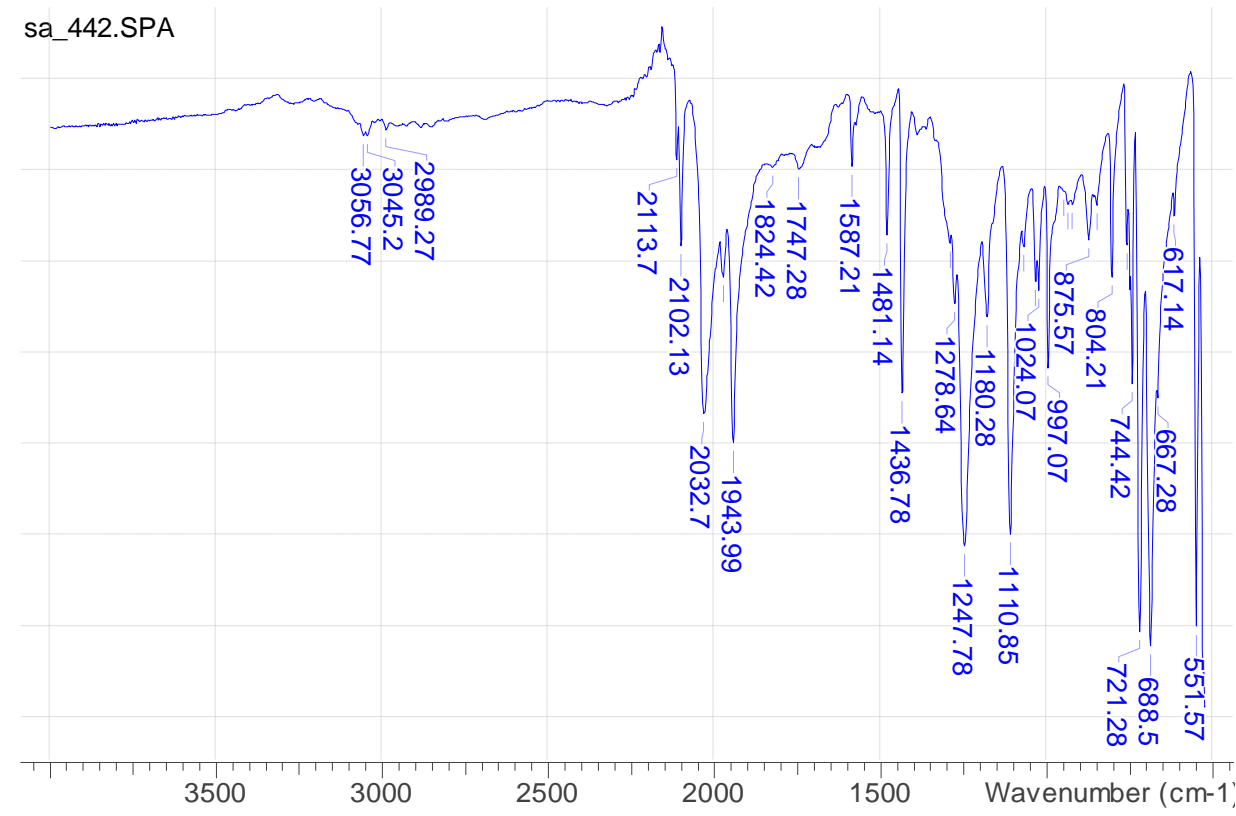

\section{RAMAN}


sa_442_a_(633_D03_x10_10s_20acc).spc

๙ิ่

$\stackrel{\sim}{\circ}$

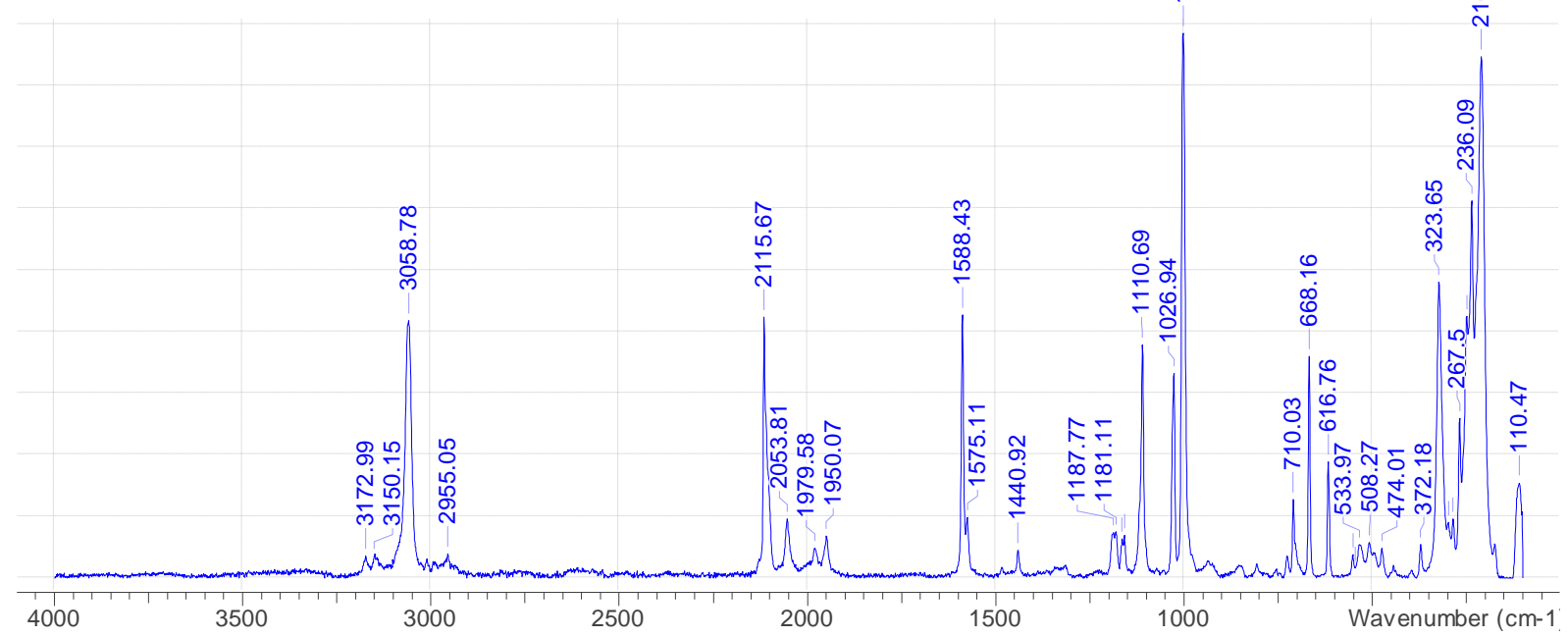

${ }^{1} \mathrm{H}$ NMR

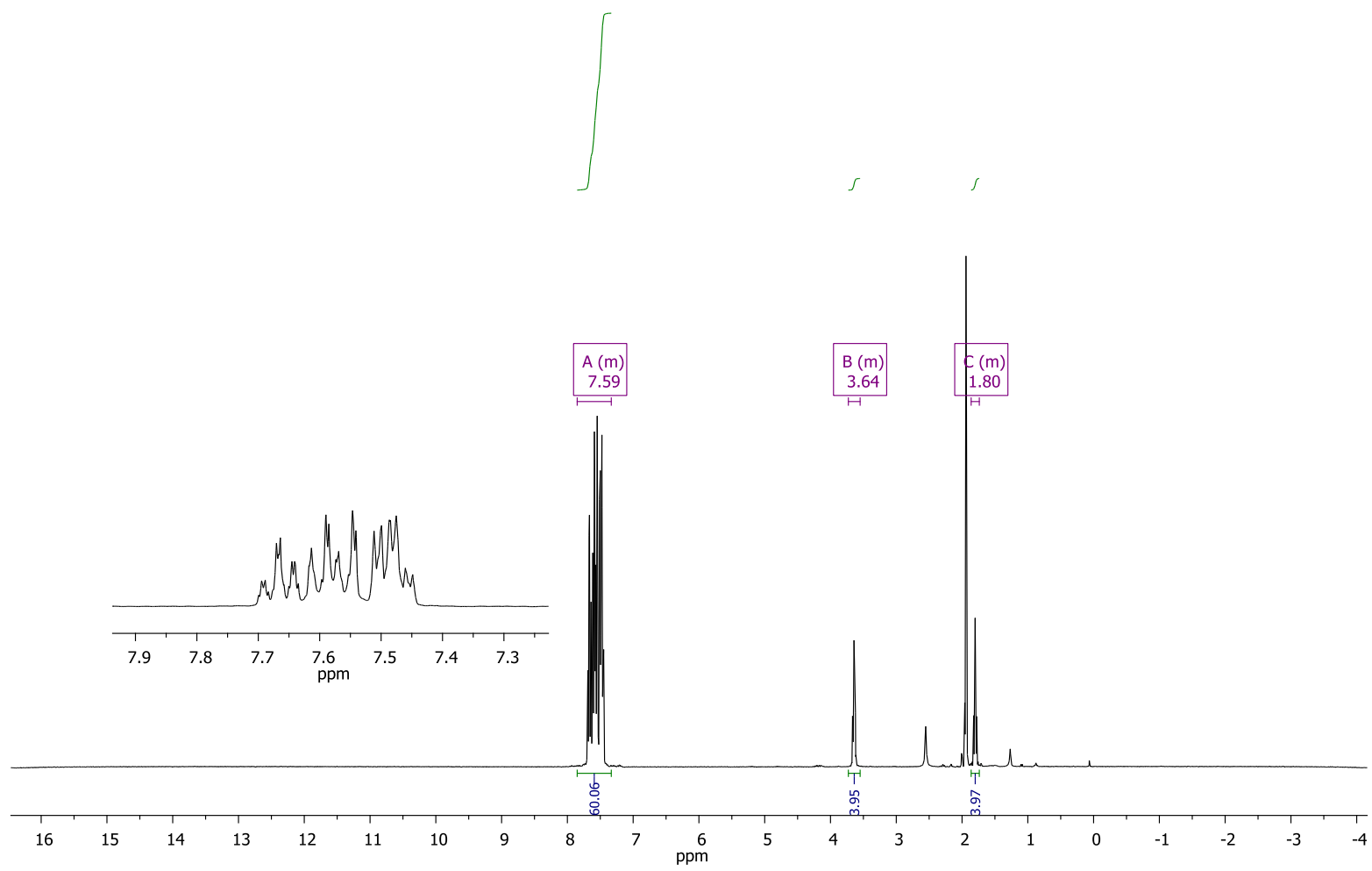




\section{${ }^{13} \mathrm{C}$ NMR}

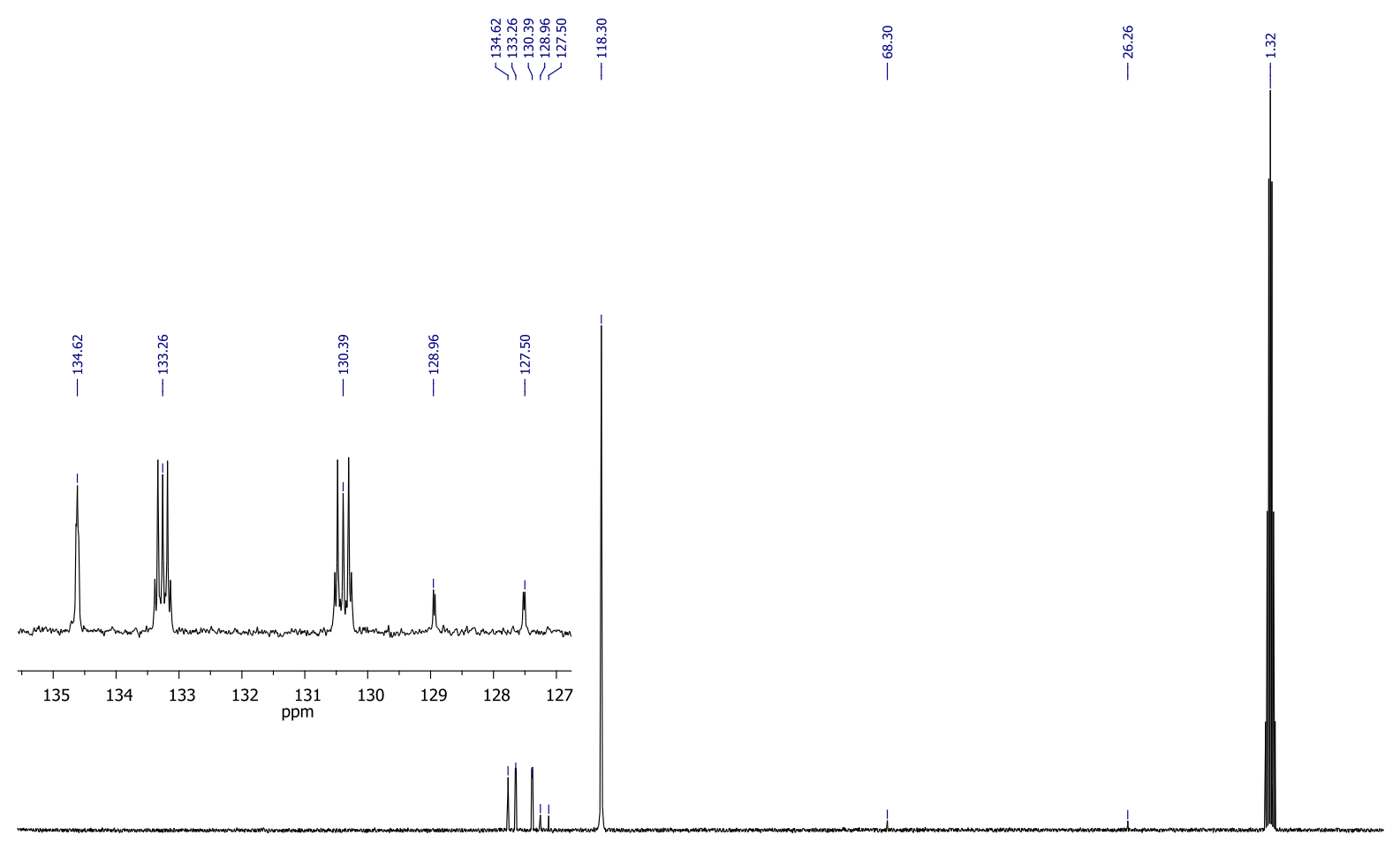

\begin{tabular}{rllllllllllllllllllllllll}
\hline 220 & 210 & 200 & 190 & 180 & 170 & 160 & 150 & 140 & 130 & 120 & 110 & 100 & 90 & 80 & 70 & 60 & 50 & 40 & 30 & 20 & 10 & 0 & -10
\end{tabular}

${ }^{31} \mathrm{P}$ NMR

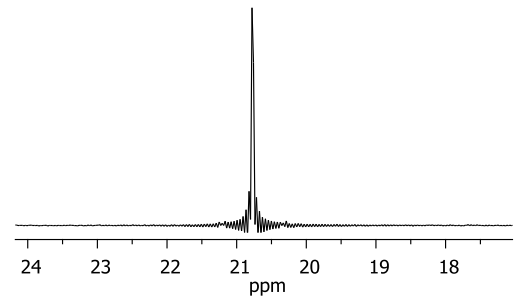

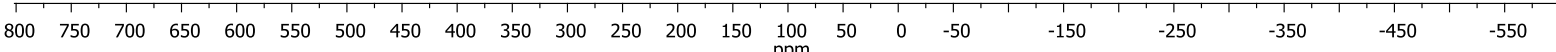




\section{References}

[1] G. M. Sheldrick, SHELXS-2013: Program for the Solution of Crystal Structures, University Of Göttingen, Germany, 2013.

[2] G. M. Sheldrick, SHELXL-2013: Program for the Refinement of Crystal Structures, University Of Göttingen, Germany, 2013.

[3] G. M. Sheldrick, SADABS Version 2, University Of Göttingen, Germany, 2004. 